\title{
EMC10 reduction in human neurons and adult mouse brain rescues cellular and behavioral deficits linked to 22q11.2 deletion
}

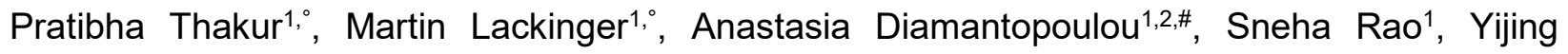
Chen $^{1,3}$, Annie Ferng ${ }^{4}$, Curt Mazur ${ }^{4}$, Holly Kordasiewicz ${ }^{4}$, Robert J. Shprintzen ${ }^{5}$, Sander Markx ${ }^{6,7}$, Bin $\mathrm{Xu}^{6,7}$, Joseph A. Gogos ${ }^{1,2,6,8,{ }^{*}}$

${ }^{1}$ Mortimer B. Zuckerman Mind Brain and Behavior Institute, Columbia University, New York, NY 10027, USA

${ }^{2}$ Department of Physiology and Cellular Biophysics, College of Physicians and Surgeons, Columbia University, New York, NY 10032, USA

${ }^{3}$ Department of Genetics and Development, Columbia University Irvine Medical Center, New York, NY 10032, USA

${ }^{4}$ Ionis Pharmaceuticals, Inc., Carlsbad, CA 92010, USA

${ }^{5}$ The Virtual Center for Velo-Cardio-Facial-Syndrome, Inc.

${ }^{6}$ Department of Psychiatry, Vagelos College of Physicians \& Surgeons, Columbia University, New York, NY 10032, USA

${ }^{7}$ Division of Molecular Therapeutics, New York State Psychiatric Institute, New York, NY 10032, USA

${ }^{8}$ Department of Neuroscience, Columbia University, New York, NY 10032, USA

\#Present address: Institute of Neurophysiology, Goethe University, Theodor-Stern-Kai 7, D-60590 Frankfurt am Main, Germany

*Correspondence: jag90@columbia.edu (J.A.G.)

These authors contributed equally to this work. 
Up-regulation of Mirta22/Emc10 is a major transcriptional effect of the 22q11.2-associated microRNA dysregulation and underlies key cellular as well as behavioral deficits. EMC10 is a component of the ER membrane complex, which promotes membrane insertion of a subset of polytopic and tail-anchored membrane proteins. Here we show that EMC10 expression is elevated in hiPSC-derived neurons from 22q11.2 deletion carriers and that reduction of EMC10 levels restores defects in neurite outgrowth and calcium signaling. Moreover, using injection of Antisense Oligonucleotides, we demonstrate that normalization of Emc10 levels in adult mouse brain rescues social memory deficits. The observations that elevated EMC10 expression is deleterious in 22q11.2 deletion carriers and that sustained elevation of EMC10 throughout the adult life can interfere with neural processes point to manipulations of EMC10 levels and downstream targets as a specific venue to ameliorate or even prevent disease progression in 22q11.2 deletion syndrome.

Adults and children with the 22q11.2 Deletion Syndrome (22q11.2DS) demonstrate cognitive, social and emotional impairments ${ }^{1-3} .22 q 11.2$ deletions are also one of the strongest genetic risk factors for schizophrenia $(\mathrm{SCZ})^{4}$. Previous work in a model of the 22q11.2 deletion, carrying a hemizygous 1.3-Mb deficiency on mouse chromosome $16[D f(16) A]$, which is syntenic to the 1.5Mb 22q11.2 deletion $\left[D f(16) A^{+/-}\right.$mice] described a distinct behavioral and cognitive profile ${ }^{5,6}$. Comprehensive RNA profiling of $D f(16) A^{+-}$mice found that postnatal elevation in the expression of the Mirta22/Emc10 gene (herein after referred to as Emc10) represents a key transcriptional effect of the 22q11.2 deletion ${ }^{7}$. Increased brain expression of Emc10 is recapitulated in $D f(16) A^{+/-}$primary neurons ${ }^{8}$ as well as in mouse models of the more common $3 \mathrm{Mb} 22 \mathrm{q} 11.2$ deletion ${ }^{9}$. Our molecular analysis of the $D f(16) A^{+/}$strain provided compelling evidence for abnormal processing of brain enriched microRNAs (miRNAs) $)^{5,7}$. The $D f(16) A^{+/-}$related miRNA dysregulation is due to (i) hemizygosity of Dgcr8, a component of the "microprocessor" complex that is essential for miRNA production ${ }^{10}$ and (ii) hemizygosity of miRNA genes residing within the 
deletion, including mir185. Reduction of the miR-185 levels and to a lesser degree of miRNAs residing outside the deletion (such as $\mathrm{miR}-485^{7}$ ) result in a de-repression of Emc10, whose expression is under the repressive control of miRNAs ${ }^{7}$. Emc10 encodes for a component of the ER membrane complex (EMC), which promotes membrane insertion and maturation of a subset of polytopic and tail-anchored membrane proteins including neurotransmitter receptors, channels, and transporters ${ }^{11-18}$. Emc10 is a prenatally biased gene with high expression in embryonic life that gradually subsides after birth ${ }^{7}$, a developmental pattern of expression conserved between mice, humans and nonhuman primates ${ }^{19}$. In that regard, the end effect of de-repression of Emc10 expression is evident primarily in postnatal life in the form of a robust and reproducible increase in Emc10 postnatal levels in Df(16) $A^{+-}$mice compared with wild-type (WT) mice ${ }^{7}$. Importantly, a Emc10 LoF mutation that leads to reduction of Emc10 levels rescues key cellular, cognitive and behavioral alterations in the $D f(16) A^{+/}$mice including abnormalities in social memory (SM $)^{19}$.

Two key issues remain unresolved towards translating these observations into potential disease-modifying therapeutic interventions. First, it remains unknown whether human EMC10 expression is also elevated in neurons from 22q11.2 deletion carriers and whether reversal of EMC10 expression leads to restoration of key morphological and functional alterations linked to 22q11.2 deletions. Second, it remains unknown whether Emc10 normalization in the adult brain is effective at reversing behavioral alterations.

To investigate whether upregulation of EMC10 is also prominent in cortical neurons from patients carrying 22q11.2 deletions (Fig. 1a), we used hiPSC lines obtained from 22q11.2DS/SCZ donors with a $3 \mathrm{Mb}$ deletion diagnosed with SCZ and healthy controls (Supplementary Table 1, Fig. S1a-d). We first derived cortical neurons out of hiPSC using an approach that combines small-molecule inhibitors to repress SMAD and WNT signaling pathways to promote CNS fate ${ }^{20}$. We confirmed the efficiency of differentiation using immunohistochemistry (IHC) and gene expression assays, which indicated the anticipated increase of TUJ1/TBR1 positive derived neurons and downregulation of embryonic stem cell marker (Fig. 1b, Fig. S1e-g). An unbiased 
evaluation of the transcriptional responses using bulk RNA sequencing on RNA collected from DIV8 differentiated human cortical neurons derived from one patient (Q6) and the corresponding healthy monozygotic twin (Q5) line revealed 4031 genes that were differentially expressed between case and control neurons (padj < 0.05, Fig. 1c). Of these, 2094 genes were significantly downregulated and 1937 genes were significantly upregulated in case compared to control neurons (Fig. 1c). GO term enrichment analysis on downregulated genes identified significantly altered biological processes centered on neurogenesis, neuronal development, and differentiation (Fig. 1d). Cellular component terms were associated with neuronal projections, junctions, and synapses. Among the upregulated genes, the GO terms enriched were related to neuronal development as well as neuronal cilia assembly and structure (Fig. 1e). Notably, we observed the expected downregulation of genes within the $22 q 11.2$ locus as well as a significant upregulation of EMC10 in patient neurons (Fig. S2). Expression of other EMC subunits (EMC14, EMC6-9) detected in our DIV8 sequencing data did not show significant differences. Further RNA and protein expression characterization confirmed the reductions in the abundance of the 22q11.2 locus residing genes DGCR8 and RANBP1 (Fig. 1f-g). We also confirmed a robust transcriptional EMC10 upregulation in human cortical neurons derived from the same Q5/Q6 pair in RNA collected from three district stages of in vitro maturation (Fig. 1h) as well as in protein extracts collected from cortical neurons (Fig. 1i). Upregulation of EMC10 was also confirmed in cortical neurons from a second, independent patient line (QR27) compared to healthy control line (Q20) (Fig. 1j). To examine whether transcriptional EMC10 upregulation is independent of the neuronal derivation method, we also generated neurons via inducible expression of Neurogenin2 (NGN2) in human iPSCs, a widely used protocol that generates within 3 weeks a robust population of excitatory neurons (NGN2-iNs) ${ }^{21-24}$ (Fig. 1k). qRT-PCR assay of EMC10 mRNA expression level in NGN2-iNs at DIV21 confirmed transcriptional EMC10 upregulation in three independent pairs of patient and sex-lage matched healthy control lines (Fig. 1I-n). Taken 
together our results highlight a reproducible and robust upregulation of EMC10 in neurons derived from patients with 22q11.2 deletions, which is independent of the derivation method.

We also examined whether 22q11.2 deletion results in abnormal processing of miRNAs in human neurons as we have previously described in animal models ${ }^{5}$. We performed parallel small RNA/miRNA sequencing on DIV8 differentiated human cortical neurons derived via SMAD/ WNT signaling inhibition from hiPSCs of the same monozygotic twin (Q5/Q6) pair used for bulk mRNA sequencing. We identified a number of mature miRNAs dysregulated in response to the 22q11.2 deletion (Fig. 2a, Fig. S3). As a validation of our approach, we observed the expected downregulation of miRNAs miR-185, miR-1286 and miR-1306 that reside in the 22q11.2 locus (three other predicted 22q11.2 miRNAs, miR-649, miR-3618 and miR-4761 were not detected in DIV8 neurons). Among miRNAs located outside the 22q11.2 region, we note downregulation of miRNAs such as miR-137 as well as miR-134 and several other members form the largest placental mammal-specific miRNA gene cluster miR379-410 (Fig. S3) that have been previously implicated in neuronal development, differentiation and function ${ }^{5,25-33}$. We used the miRNA-target interaction network tool miRNet $2.0^{34}$ to perform target enrichment and network analysis for the dysregulated miRNAs and performed GO term enrichment analysis on this target interaction network. Affected biological processes were prominently centered on cell division and intracellular protein transport (Fig. 2b) whereas cellular components were associated with the nucleus and the perinuclear region (endoplasmic reticulum and Golgi apparatus) of the cytoplasm (Fig. 2c). In addition to 22q11.2 deletion region miRNAs, lower abundance of miRNAs in cases is likely due to haploinsufficiency of the DGCR8 gene and is expected to result in upregulation of target genes. Intersection of predicted targets of downregulated miRNAs and up-regulated DEGs identified predicted targets of downregulated miRNAs (Fig. 2d). Notably, functional annotation revealed that predicted targets of downregulated miRNAs include genes that modulate neuronal development and are associated with GO terms such as endoplasmic reticulum and endomembrane system of neurons (Fig. 2e-f). 
We have previously shown that upregulation of the murine orthologue of Emc10 is primarily due to downregulation of miR-185 and to a lesser degree of miR-485 ${ }^{7}$. Both conserved and non-conserved binding sites at the 3'UTR of human EMC10 are predicted in silico for both miRNAs (Fig. S4a). Consistently, the observed upregulation in the levels of EMC10 gene is accompanied by a robust reciprocal decrease in the levels of the miRNA precursor of miR-185 at DIV8 as indicated both by our miRNA sequencing analysis (Fig. 2a) and follow up qRT-PCR assays (Fig. 2g). miRNA precursor of miR-485 showed a more modest non-significant reduction in its abundance (Fig. 2g). Notably, overexpression of miR-185 and miR-485 using miRNA mimics in human cortical neurons at DIV10 resulted in reduction of EMC10 expression levels in both the healthy control (Fig. 2h) and patient line (Fig. 2i). Furthermore, inhibition of endogenous miR-185 and miR-485 in the control line by using specific miRNA inhibitors increased EMC10 expression level (Fig. 2j) confirming the predicted conserved functionality of miR-185 and miR-485 miRNA binding sites in EMC10. It is worth noting that in addition to miR-185, non-conserved binding sites at the 3'UTR of human EMC10 are predicted in silico for two additional downregulated miRNAs residing within the 22q11.2 locus, miR-1286 and miR-1306 (Supplementary Table 2). Indeed, EMC10 is one of the only 104 upregulated genes that are predicted to carry binding sites at the 3'UTR of all three downregulated 22q11.2 miRNAs (Fig. S4b-c). The functionality of these miRNA binding sites in EMC10 and whether they contribute to the observed elevation of its expression in human neurons remains to be determined. Taken together our results confirm that miRNA dysregulation emerges in human neurons as a result of the $22 q 11.2$ deletion and in turn drives misexpression of genes primarily involved in intracellular membrane and protein traffickingrelated processes required for neuronal development and maturation. Among them, EMC10 represents a major downstream effector of the 22q11.2-linked miRNA dysregulation.

To investigate the relevance of EMC10 de-repression in the development and function of patient neurons, we generated derivatives of the Q6 patient hiPSC line carrying either heterozygous (Q6/EMC10 $\left.{ }^{\mathrm{HET}}\right)$ or homozygous (Q6/EMC10 ${ }^{\mathrm{HOM}}$ ) EMC10 LoF mutations using 
standard CRISPR/Cas9 editing approaches (Fig. S5a). Mutations were confirmed by sequencing (Fig. S5a, lower panel) and karyotyping confirmed normal chromosome complement (Fig. S5b). We confirmed reduced expression levels of 22q11.2 genes (DGCR14 and LZTR1) in both derivative iPSC lines whereas stem-cell marker NANOG and OCT4 were equally expressed in all lines (Fig. S5c-f). EMC10 mRNA and protein levels were reduced by $~ 50 \%$ in the Q6/EMC10 hiPSC line and abolished in the Q6/EMC10 ${ }^{\mathrm{HOM}}$ line (Fig. 3a, Fig. S5g). Additional characterization of hiPSC-derived derived NGN2-iNs (Fig. 3b), also conclusively demonstrated a reduction (Q6/EMC10 ${ }^{\mathrm{HET}}$ ) or elimination (Q6/EMC10 ${ }^{\mathrm{HOM}}$ ) of EMC10 mRNA (Fig. 3c).

$D f(16) A^{+/-}$mice show impaired formation of dendrites in their brains, which are faithfully recapitulated in primary neuronal cultures and are partially reversed by reduction of Emc10 levels ${ }^{7}$. We asked whether impaired dendritic formation is also observed in human neuronal cultures from patients with 22q11.2 deletions and whether reduction of EMC10 levels could prevent such morphological alterations during neuronal maturation. We employed monolayer neuronal cultures of NGN2-iNs. Neuronal cells were fixed at DIV21 of differentiation, traced and key indices of dendritic architecture were quantified (see method). Our analysis confirmed a reduced dendrites complexity in mutant neurons as reflected in total neuronal length, the number of branch points/cell and the total number of dendrite/cells (Fig. $\mathbf{3 d - g}$ ). The number of primary dendrites per cell were unchanged (Fig. 3h). Importantly, we found that reduction or elimination of EMC10 expression restored to WT levels neuronal length and branchpoints. It should be noted that the beneficial effect of complete elimination of EMC10 expression is consistent with previous findings indicating that lack of EMC10 does not compromise EMC assembly ${ }^{35}$ and implying an auxiliary or modulatory role of EMC10 in the EMC function.

Our previous evaluation of $\mathrm{Ca}^{2+}$ homeostasis perturbations caused by $22 \mathrm{q} 11.2$ deletions using $\mathrm{Ca}^{2+}$ imaging on primary neurons from $\mathrm{Df}(16) A^{+/}$mice revealed a significantly lower amplitude of $\mathrm{Ca}^{2+}$ elevation following $\mathrm{KCl}$ evoked depolarization ${ }^{8}$. This impairment was recently replicated in human cortical neurons from patients with $22 q 11.2$ deletions ${ }^{36}$ and shown to be 
partially restored by exogenous expression of $D G C R 8$, indicating a potential role of miRNA dysregulation. Using the green-fluorescent calcium indicator Fluo-4 and time-lapse microscopy we confirmed a decrease in the amplitude of $\mathrm{Ca}^{2+}$ rise following $\mathrm{KCl}$ evoked depolarization, in patient (Q6) derived NGN2-iNs at DIV37/38 compared to the healthy twin (Q5) (Fig. 3i-I). We asked whether reduction of EMC10 levels could reverse such alterations. Notably, the observed defect in $\mathrm{Ca}^{2+}$ signaling were reversed in both Q6/EMC10 ${ }^{\mathrm{HET}}$ and Q6/EMC10 HOM NGN2-iNs as demonstrated by the increased amplitude of $\mathrm{Ca}^{2+}$ rise following depolarization (Fig. $\left.\mathbf{3 k}-\mathbf{I}\right)$. The observation that reduction of EMC10 levels fully restores the $\mathrm{Ca}^{2+}$ signaling deficits observed in patient neurons suggests that miRNA-dependent elevation in EMC10 levels may interfere with one or more sources of intracellular $\mathrm{Ca}^{2+}$ and a wide range of calcium-dependent processes. Overall, our analysis of neurons from 22q11.2 deletion carriers indicate that elevation of EMC10 expression disrupts their maturation and function and together with similar observations in animal models support normalization of EMC10 expression as a disease-modifying intervention.

Toward this end, we investigated whether normalization of Emc10 levels in the brain of adult (2-3-month-old) $D f(16) A^{+-}$mice via transient injection of single stranded Antisense Oligonucleotides (ASOs) is effective at reversing key cognitive alterations ${ }^{5,19}$. We first screened over 300 in silico designed chimeric 2'-O-methoxyethyl/DNA gapmer. ASOs were generated and screened for Emc10 mRNA reduction in 4T1 cells via electroporation (Fig. 4a). Lead ASOs were then confirmed in a dose-response assay (Fig. 4b), including the lead ASO (1081815, herein referred to as Emc10 ${ }^{\mathrm{ASO}}$ ), which targets intron 2 of mouse Emc10 (Fig. 4c). Emc10 ASO1 was selected for subsequent studies, as it was effective in lowering Emc10 expression both in vitro and in vivo. Specifically, following transient intracerebral ventricular (ICV) injection in the posterior ventricle of 8 weeks old WT mice, Emc10 ${ }^{\text {ASO1 }}$ effectively suppressed the levels of Emc10 mRNA (Fig. 4d) and protein (Fig. 4e) in both left and right hippocampus compared to WT mice treated with a negative $\mathrm{Ctrl}^{\mathrm{ASO} 1}$ without complementarity in the mouse. Analysis of Gfap and Aif1 expression did not reveal any changes (Fig. 4f) suggesting lack of astroglial and 
microglial activation upon Emc10 $10^{\mathrm{SO} 1}$ injection. Emc10 ${ }^{\mathrm{ASO}}$ injected mice showed normal gait and no signs of behavioral toxicity. IHC analysis using an antibody that selectively recognizes the phosphorothioate backbone verified a robust diffusion primarily in hippocampus and to a lesser degree in surrounding brain areas. Colocalization with the neuronal marker NeuN and glial fibrillary acidic protein (GFAP) confirmed accumulation in hippocampal neurons as well as GFAPexpressing astrocytes (Fig. $\mathbf{4 g})$.

Analysis of $D f(16) A^{+/}$mice treated by intraventricular injection at 8 weeks of age showed that Emc10 ${ }^{\mathrm{ASO}}$ effectively lowered hippocampal Emc10 mRNA to nearly WT levels 3 weeks postinjection resulting in normalization of Emc10 expression (Fig. 4h, left panel). By contrast, consistent with the pattern of ASO distribution, we did not observe a significant reduction of Emc10 expression levels in the prefrontal cortex (PFC) of $D f(16)^{+/}$mice treated with Emc10 $0^{\mathrm{ASO} 1}$ (Fig 4h, right panel). In addition to targeted assays, we performed bulk RNA-sequencing analysis of $\mathrm{Ctrl}^{\mathrm{ASO} 1}$ and Emc10 ${ }^{\mathrm{ASO} 1}$-treated $\mathrm{Df}(16) A^{+/}$mice and WT littermates to evaluate the effect of Emc10 ${ }^{\mathrm{ASO} 1}$ treatment on the hippocampal transcriptome profile. In the $\mathrm{Ctrl}{ }^{\mathrm{ASO} 1}$ treated group (Fig. $\mathbf{4 i}$, left panel), we observe the tripartite differential gene expression signature characteristic of $D f(16) A^{+/-}$mice: upregulation of Emc10 and non-coding RNAs (pri-forms of miRNAs and long non-coding RNAs (Fig. 4i, left panel and inset, Log2Fold Change $=0.5$ ) as well as the expected downregulation of genes included within the $D f(16) A$ deficiency. In the Emc10 ASO1 treated group (Fig. 4i, right panel and inset), Emc10 is no longer upregulated in $D f(16) A^{+/}$mice while noncoding RNAs remain upregulated, and genes included in the deficiency are robustly downregulated. Apart from Emc10, seven other genes (Mir9-3hg, Plxnd1, Cd68, Mir22hg, Gm28439, Adgre1, and Tnn) are significantly upregulated in $D f(16) A^{+/}$mice in the $\mathrm{Ctrl}^{\mathrm{ASO} 1}$ but not in the Emc10 ${ }^{\mathrm{ASO} 1}$-treated group (Supplementary Table S4). We used the Bowtie mapping tool ${ }^{37}$ to align short sequencing reads on both genomic and transcript sequence to assess whether these expression changes represent potential off-target effects of the Emc10 ASO1 in the mouse transcriptome. Emc10 ${ }^{\mathrm{ASO}}$ exclusively aligned with full complementarity to an intronic region in the 
Emc10 gene (Fig. 4c) providing additional support for high target specificity. The observed changes might represent downstream effects of Emc10 level reduction or reflect expression variability due to low expression levels of the upregulated genes.

We examined the effects of Emc10 ${ }^{\mathrm{ASO}}$-mediated Emc10 reduction in adult brain on behavioral performance. 8-week-old $D f(16) A^{+/-}$mice and WT littermates were treated by ICV injection of $\mathrm{Emc}^{\mathrm{ASO} 1}$ and $\mathrm{Ctr} \mathrm{ASO}^{\mathrm{AS}}$ and tested 3 weeks later in assays for SM and contextual fear memory, two hippocampal-dependent cognitive domains robustly and reproducibly affected in $D f(16) A^{+/-}$mice. Deficits in social cognition are present in individuals with $22 q 11.2$ deletions and although rodents do not display all features of human social cognition, use of rodent tasks that evaluate SM can serve as a useful proxy of the human condition. $D f(16) A^{+/}$mice display a markedly impaired $\mathrm{SM}^{6}$ which is fully restored by genetic reduction of Emc10 levels ${ }^{19}$. In SM assays, $D f(16) A^{+-}$mice treated with $\mathrm{Ctr}^{\mathrm{ASO} 1}$ showed the expected deficits in SM as reflected in the sustained high interaction time with the reintroduced familiar juvenile mouse. By contrast, $D f(16) A^{+-}$mice injected with Emc10 $10^{\mathrm{ASO} 1}$ had significantly improved performance to levels indistinguishable from Emc10 ${ }^{\mathrm{ASO} 1}$-treated WT littermates consistent with improvement of function arising from adult restoration of Emc10 levels (Fig. 5a). Specifically, upon reintroduction of a familiar juvenile mouse Emc10 ASO1-treated $D f(16) A^{+/}$mice showed a strong reduction in social interaction, indicative of intact SM, comparable to Emc10 ASO1-treated WT littermates and significantly different from $\mathrm{Df}(16) \mathrm{A}^{+/-}$mice treated with $\mathrm{Ctr} \mathrm{ISO}^{\mathrm{ASO}}$ [three-way ANOVA for trial $\mathrm{x}$ genotype $x$ treatment interaction matching by trial: $F(1,38)=9.393, P=0.0040$; post hoc Tukey, $\mathrm{P}=0.0114]$. The intact $\mathrm{SM}$ of the Emc10 $\mathrm{ASO}^{\text {-treated }} \mathrm{Df}(16) A^{+/}$mice was further evident in analysis of difference score (Fig. 5b) compared to the $\mathrm{Ctrl}^{\mathrm{ASO} 1}$-treated $\mathrm{Df}(16) \mathrm{A}^{+/}$mice (two-way ANOVA for genotype treatment $x$ interaction $F(1,38)=9.369, P=0.0040$; post hoc Tukey, $P=0.0004)$. Rescue was observed in both sexes and no significant differences were seen in treatment across sexes [three-way ANOVA of trial 2 sex $x$ genotype $x$ treatment: $F(1,34)=$ $0.002071, \mathrm{P}=0.9640]$. In control experiments, no effects of genotype or treatment were observed 
upon reintroduction of a novel juvenile mouse in trial 2 (Fig. 5c, d), strongly indicating that SM deficits are not driven by a simple task fatigue.

In contrast to SM deficits, we have previously shown that genetic reduction of Emc10 levels in $D f(16) A^{+/-}$mice resulted in only partial restoration of deficits in contextual fear memory ${ }^{19}$. We confirmed the fear memory deficit in $D f(16) A^{+/}$mice treated with a Ctrl ${ }^{A S O 1}$ compared with WT littermates. Analysis of Emc10 ${ }^{\mathrm{ASO}}$-treated mice revealed that $\mathrm{Emc10}{ }^{\mathrm{ASO} 1}$ treatment was not sufficient to fully rescue the learning deficit in $D f(16) A^{+/-}$mice to WT levels (Fig. 5e, right panel). However, there was a small improvement in fear memory of Emc10ASO1-treated $D f(16) A^{+/}$mice, since these mice did not differ significantly from the Emc10 ASO1-treated WT littermates. Taken together with similar results from our previous genetic rescue assays ${ }^{19}$, this finding likely indicates a more limited role of Emc10 upregulation in this cognitive deficit rather than requirement for additional treatment time or for earlier onset of Emc10 normalization to completely restore the underlying neural circuitry.

To evaluate the consistency and reproducibility of the behavioral rescue, we generated additional in silico designed ASOs and screened them for Emc10 mRNA reduction in vivo (Fig. S6a-c). One of these ASOs (1466182, herein referred to as Emc10 ${ }^{\mathrm{ASO}}$ ), which targets intron 1 of mouse Emc10 (Fig 6a) was selected as the lead ASO for the replication analysis based on its efficacy in reducing Emc10 levels, wide distribution pattern, as well as the lack of any signs of astroglial/microglial activation or behavioral toxicity (see methods). IHC analysis showed robust distribution in both hippocampus (Fig. 6b, top panel) and PFC (Fig. 6b, middle panel) as well as diffusion into both neuronal and non-neuronal cells (Fig. 6b, bottom panel). Consistent with the widespread distribution, qRT-PCR analysis of $D f(16) A^{+/-}$mice treated by intraventricular injection at 8 weeks of age showed that Emc10 ASO2 effectively lowered Emc10 mRNA to nearly WT levels 3 weeks post-injection resulting in widespread normalization of Emc10 expression in the hippocampus (Fig. 6c), PFC (Fig. 6d), and somatosensory cortex (Fig. 6e). 
To study the effects of Emc10 ${ }^{\mathrm{ASO}}$-mediated Emc10 reduction in adult brain on behavioral performance, 8-week-old $D f(16) A^{+/}$male mice and WT littermates were treated by ICV injection of $\mathrm{Emc} 10^{\mathrm{ASO} 2}$ and $\mathrm{Ctrl} \mathrm{ASO}^{\mathrm{ASO}}$ and tested 3 weeks later in SM assay. $\mathrm{Df}(16) A^{+/-}$mice injected with Emc10 ${ }^{\mathrm{ASO} 2}$ had significantly improved performance to levels indistinguishable from Emc10 ASO2_. treated WT littermates consistent with improvement of function arising from adult restoration of Emc10 levels (Fig. 6f). Upon reintroduction of a familiar juvenile mouse, Emc10 ${ }^{\mathrm{ASO}}$-treated $D f(16) A^{+/-}$mice showed a strong reduction in social interaction, indicative of intact SM, comparable to $\mathrm{Emc10}{ }^{\mathrm{ASO}}$-treated WT littermates and significantly different from $\mathrm{Df}(16) \mathrm{A}^{+/-}$mice treated with $\mathrm{Ctrl}^{\mathrm{ASO} 2}$ [three-way ANOVA for trial $\mathrm{x}$ genotype $\mathrm{x}$ treatment interaction matching by trial: $\mathrm{F}(1,26)$ $=35.74$; post hoc Tukey Emc10 ${ }^{\mathrm{ASO} 2}$ vs $\left.\mathrm{Ctr}\right|^{\mathrm{ASO} 2}$ treated WT, $\mathrm{P}=0.0255$ ]. These data were further supported by difference score analysis (Fig. 6g).

The advancement of the present study is twofold. First, we show that human EMC10 expression is elevated in neurons from $22 q 11.2$ deletion carriers and that effective knockdown to near WT levels or even complete depletion of EMC10 leads to restoration of key morphological and functional alterations in neuronal maturation emerging due to $22 q 11.2$ deletions. Second, we show that normalization of Emc10 levels in adult mouse brain is effective in rescuing specific behavioral deficits strongly suggesting that for a subset of 22q11.2-associated behavioral deficits it is the sustained elevation of EMC10 throughout the adult life that interferes with the underlying neural processes rather than an irreversible impact on brain maturation during early development. These findings strengthen the link between EMC10 upregulation and the behavioral dysfunction observed in 22q11.2 deletion carriers. EMC targets are typically multi-transmembrane domain (TMD) proteins ${ }^{17,18,38}$ that contain low-hydrophobicity TMDs which are hard to insert into ER membrane and thus require the aid of EMC as a membrane insertase ${ }^{11,12,17}$. Identification of EMC clients whose biogenesis, trafficking and membrane insertion are affected by EMC10 upregulation could provide further insights into the beneficial effects of the restoration in EMC10 expression and help establish a link between such downstream targets and circuit/behavioral dysfunction 
emerging due to $22 q 11.2$ deletions. It is attractive to speculate that different EMC10 upregulationlinked phenotypes and their developmental requirements may be driven, individually or in combinations, by distinct downstream targets.

Our results also have potential implications for therapeutic interventions aiming to manipulate Emc10 expression, activity as well as downstream targets. First, identification of neurotransmitter receptors, channels, and transporters whose membrane trafficking are affected by EMC10 upregulation can guide efforts to develop treatments for specific 22q11.2 deletion symptoms. Second, for genetically driven and postnatally reversible symptoms of neurodevelopmental disorders (NDDs) emerging treatments include reduction of culprit gene expression via sequence-specific knockdown of mRNA transcripts ${ }^{39}$. Use of ASOs offers one such potential venue, dictated by their demonstrated efficacy as a therapeutic modality in preclinical models ${ }^{40-45}$ and clinical studies of NDDs or neurodegenerative disorders ${ }^{46-48}$. Towards this goal, future experiments will determine whether ASO-mediated normalization of adult Emc10 levels restores additional $22 q 11.2$ behavioral alterations. A common challenge in efforts to employ gene-knockdown therapies for dosage-sensitive genes in NDDs is restricting target gene expression within optimal levels to avoid toxicity due to target hyper-knockdown ${ }^{49}$. Regarding EMC10, a large number of relatively rare LoF variants or potentially damaging missense variants have been identified in the human $E M C 10$ gene among likely healthy individuals in gnomAD ${ }^{50}$ which is depleted of individuals known to be affected by severe NDDs. Taken together with our previous analysis of heterozygous Emc10 knockout mice ${ }^{19}$, these observations strongly indicate that $50 \%$ reductions in Emc10 levels are well tolerated at the level of the organism. The highest dose for both Emc10-specific ASOs used in mutant $D f(16) A^{+-}$mice was limited to $\sim 300 \mu \mathrm{g}$ and reduced Emc10 mRNA to either normal or slightly below normal (10-20\% of WT) expression while attaining full behavioral rescue. While higher dose may be required to ameliorate other behavioral

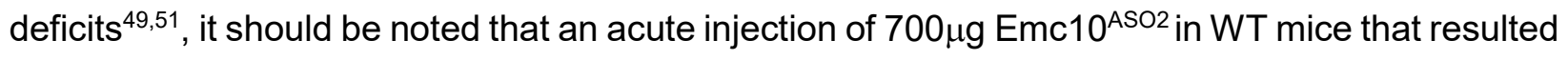


in $\sim 50 \%$ reduction of Emc10 levels (Fig. S6) did not cause secondary cellular and behavioral toxicity. Thus, available evidence indicates that therapeutically effective ASO-mediated normalization of EMC10 levels can be up- or down-titrated within an unequivocally safe range. The effect of further reductions of EMC10 levels remains unknown. Homozygous EMC10 mutations were recently reported in consanguineous families of Middle Eastern origin with developmental delay, seizures and dysmorphic features ${ }^{51}$ suggesting that EMC10 levels both above or below an optimal range may be deleterious. Nevertheless, increased consanguinity and lack of data on the prevalence of deleterious EMC10 mutations among healthy individuals in these populations complicate interpretation of these findings. Replication in genetically heterogeneous populations is necessary to evaluate the potential pathogenicity and penetrance of homozygous EMC10 mutations especially in relation to gene-based treatments.

Overall, our results support a key contribution of EMC10 and EMC-dependent membrane protein trafficking in mediating the effects of 22q11.2 deletions, point to manipulations of EMC10 expression and activity as well as of downstream targets as an attractive alternative or augmentation of currently available treatments and highlight a broad temporal window of therapeutic opportunity for the 22q11.2 deletion-associated cognitive and behavioral symptoms. 


\section{Acknowledgments}

We thank Dr. Huynh-Hao Bui and Andrew Watt for design and identification of ASOs, Mark Andrade and the lonis synthesis group for ASO synthesis, and Dr. Mark Graham for ASO study design and feedback. We thank Naoko Haremaki for genotyping and maintaining the mouse colony. We thank Panagiota Apostolou for her help with the calcium imaging setup. We thank Yan Sun and Vivian Zhu for the hiPSC line validation and maintenance. We thank Barbara Corneo and the Columbia Stem Cell Core Facility for the Q1, Q2, Q5 and Q6 hiPSC lines generation. Biosamples of Q20 and Q27 hiPSCs were obtained from NIMH Repository \& Genomics Resource. We thank Linda Brzustowicz and Bill Manley from the Rutgers University and the staff members at RUCDR. Data and biomaterials generated in Study 125/Site 393 were funded by a NIMH grant to Dr. Herb Lachman (MH087840: Analysis of Glutamatergic Neurons Derived from PatientSpecific iPS Cells). The co-investigators on this grant included Dr. Deyou Zheng and Dr. Reed Carroll from the Albert Einstein College of Medicine. Patients and controls were recruited at the Albert Einstein College of Medicine and at the Child Psychiatry Branch, NIMH, directed by Dr. Judith L. Rapoport. We thank all participating subjects and their families for their contributions. This work was supported by National Institute of Mental Health Grant (2R01MH097879) and a Columbia University Translational Therapeutics Pilot Award to J.A.G.

\section{Author Contributions}

P.T. contributed to the design of the in vivo ASO screening and performed the ASO injections/surgeries as well as mouse related RNA and protein expression, immunohistochemistry and behavioral assays and data analysis. M.L. contributed to the design and performed the human neuron-related expression and phenotypic assays, the mouse protein expression assays and contributed to the implementation and analysis of mouse fear conditioning assays. A.D. contributed to the design and implementation of the in vivo ASO screening and behavioral data acquisition. SR analyzed mouse related RNA sequencing data. Y.C. contributed to mouse related 
immunohistochemistry assays. A.F., C.M, and H.K. contributed to the identification and characterization of the lead ASOs. B.X., contributed to the generation and initial characterization of human iPSC lines as well as the design of the human neuron-related assays. R.J.S provided patient referrals. S.M. contributed to the generation and initial characterization of human iPSC lines. J.A.G contributed to the conception, design and supervision of the study; P.T., M.L., S.R., B.X., S.M., and J.A.G. contributed to the preparation of the manuscript.

\section{Conflict of interest}

The authors declare that they have no conflict of interest. 


\section{References}

$1 \quad$ Morrison, S. et al. Cognitive deficits in childhood, adolescence and adulthood in 22q11.2 deletion syndrome and association with psychopathology. Transl Psychiatry 10, 53, doi:10.1038/s41398-020-0736-7 (2020).

$2 \quad$ Woodin, M. et al. Neuropsychological profile of children and adolescents with the 22q11.2 microdeletion. Genet Med 3, 34-39, doi:10.1097/00125817-200101000-00008 (2001).

3 McCabe, K. L. et al. Divergent patterns of social cognition performance in autism and 22q11.2 deletion syndrome (22q11DS). J Autism Dev Disord 43, 1926-1934, doi:10.1007/s10803-012-1742-2 (2013).

$4 \mathrm{Xu}, \mathrm{B}$. et al. Strong association of de novo copy number mutations with sporadic schizophrenia. Nat Genet 40, 880-885, doi:10.1038/ng.162 (2008).

5 Stark, K. L. et al. Altered brain microRNA biogenesis contributes to phenotypic deficits in a 22q11-deletion mouse model. Nat Genet 40, 751-760, doi:10.1038/ng.138 (2008).

6 Piskorowski, R. A. et al. Age-Dependent Specific Changes in Area CA2 of the Hippocampus and Social Memory Deficit in a Mouse Model of the 22q11.2 Deletion Syndrome. Neuron 89, 163-176, doi:10.1016/j.neuron.2015.11.036 (2016).

$7 \quad$ Xu, B., Hsu, P. K., Stark, K. L., Karayiorgou, M. \& Gogos, J. A. Derepression of a neuronal inhibitor due to miRNA dysregulation in a schizophrenia-related microdeletion. Cell 152, 262-275, doi:10.1016/j.cell.2012.11.052 (2013).

8 Sun, Z., Williams, D. J., Xu, B. \& Gogos, J. A. Altered function and maturation of primary cortical neurons from a 22q11.2 deletion mouse model of schizophrenia. Transl Psychiatry 8, 85, doi:10.1038/s41398-018-0132-8 (2018).

9 Saito, R. et al. Comprehensive analysis of a novel mouse model of the 22q11.2 deletion syndrome: a model with the most common 3.0-Mb deletion at the human $22 q 11.2$ locus. Transl Psychiatry 10, 35, doi:10.1038/s41398-020-0723-z (2020).

10 Gregory, R. I. et al. The Microprocessor complex mediates the genesis of microRNAs. Nature 432, 235-240, doi:10.1038/nature03120 (2004).

11 Guna, A., Volkmar, N., Christianson, J. C. \& Hegde, R. S. The ER membrane protein complex is a transmembrane domain insertase. Science 359, 470-473, doi:10.1126/science.aao3099 (2018).

12 Chitwood, P. J., Juszkiewicz, S., Guna, A., Shao, S. \& Hegde, R. S. EMC Is Required to Initiate Accurate Membrane Protein Topogenesis. Cell 175, 1507-1519 e1516, doi:10.1016/j.cell.2018.10.009 (2018).

13 Richard, M., Boulin, T., Robert, V. J., Richmond, J. E. \& Bessereau, J. L. Biosynthesis of ionotropic acetylcholine receptors requires the evolutionarily conserved ER membrane complex. Proc Natl Acad Sci U S A 110, E1055-1063, doi:10.1073/pnas.1216154110 (2013).

14 Satoh, T., Ohba, A., Liu, Z., Inagaki, T. \& Satoh, A. K. dPob/EMC is essential for biosynthesis of rhodopsin and other multi-pass membrane proteins in Drosophila photoreceptors. Elife 4, doi:10.7554/eLife.06306 (2015).

15 Bircham, P. W. et al. Secretory pathway genes assessed by high-throughput microscopy and synthetic genetic array analysis. Mol Biosyst 7, 2589-2598, doi:10.1039/c1mb05175j (2011).

16 Louie, R. J. et al. A yeast phenomic model for the gene interaction network modulating CFTR-DeltaF508 protein biogenesis. Genome Med 4, 103, doi:10.1186/gm404 (2012).

17 Shurtleff, M. J. et al. The ER membrane protein complex interacts cotranslationally to enable biogenesis of multipass membrane proteins. Elife 7, doi:10.7554/eLife.37018 (2018). 
18 Tian, S. et al. Proteomic Analysis Identifies Membrane Proteins Dependent on the ER Membrane Protein Complex. Cell Rep 28, 2517-2526 e2515, doi:10.1016/j.celrep.2019.08.006 (2019).

19 Diamantopoulou, A. et al. Loss-of-function mutation in Mirta22/Emc10 rescues specific schizophrenia-related phenotypes in a mouse model of the 22q11.2 deletion. Proc Natl Acad Sci U S A 114, E6127-E6136, doi:10.1073/pnas.1615719114 (2017).

20 Qi, Y. et al. Combined small-molecule inhibition accelerates the derivation of functional cortical neurons from human pluripotent stem cells. Nat Biotechnol 35, 154-163, doi:10.1038/nbt.3777 (2017).

21 Zhang, Y. et al. Rapid single-step induction of functional neurons from human pluripotent stem cells. Neuron 78, 785-798, doi:10.1016/j.neuron.2013.05.029 (2013).

$22 \mathrm{Yi}, \mathrm{F}$. et al. Autism-associated SHANK3 haploinsufficiency causes Ih channelopathy in human neurons. Science 352, aaf2669, doi:10.1126/science.aaf2669 (2016).

23 Ho, S. M. et al. Rapid Ngn2-induction of excitatory neurons from hiPSC-derived neural progenitor cells. Methods 101, 113-124, doi:10.1016/j.ymeth.2015.11.019 (2016).

24 Hui Pak, C. H. et al. Rapid generation of functional and homogeneous excitatory human forebrain neurons using Neurogenin-2 (Ngn2). Protoc. Exch. (2018).

25 Siegert, S. et al. The schizophrenia risk gene product miR-137 alters presynaptic plasticity. Nat Neurosci 18, 1008-1016, doi:10.1038/nn.4023 (2015).

26 Thomas, K. T. et al. Inhibition of the Schizophrenia-Associated MicroRNA miR-137 Disrupts Nrg1alpha Neurodevelopmental Signal Transduction. Cell Rep 20, 1-12, doi:10.1016/j.celrep.2017.06.038 (2017).

27 Schratt, G. M. et al. A brain-specific microRNA regulates dendritic spine development. Nature 439, 283-289, doi:10.1038/nature04367 (2006).

28 Fiore, R. et al. Mef2-mediated transcription of the miR379-410 cluster regulates activitydependent dendritogenesis by fine-tuning Pumilio2 protein levels. EMBO J 28, 697-710, doi:10.1038/emboj.2009.10 (2009).

29 Rago, L., Beattie, R., Taylor, V. \& Winter, J. miR379-410 cluster miRNAs regulate neurogenesis and neuronal migration by fine-tuning $\mathrm{N}$-cadherin. EMBO J 33, 906-920, doi:10.1002/embj.201386591 (2014).

30 Lackinger, M. et al. A placental mammal-specific microRNA cluster acts as a natural brake for sociability in mice. EMBO Rep 20, doi:10.15252/embr.201846429 (2019).

31 Gardiner, E. et al. Imprinted DLK1-DIO3 region of $14 q 32$ defines a schizophreniaassociated miRNA signature in peripheral blood mononuclear cells. Mol Psychiatry 17, 827-840, doi:10.1038/mp.2011.78 (2012).

32 Tomasello, U. et al. miR-137 and miR-122, two outer subventricular zone non-coding RNAs, regulate basal progenitor expansion and neuronal differentiation. Cell Rep 38, 110381, doi:10.1016/j.celrep.2022.110381 (2022).

33 Whipple, A. J. et al. Imprinted Maternally Expressed microRNAs Antagonize Paternally Driven Gene Programs in Neurons. Mol Cell 78, 85-95 e88, doi:10.1016/j.molcel.2020.01.020 (2020).

34 Chang, L., Zhou, G., Soufan, O. \& Xia, J. miRNet 2.0: network-based visual analytics for miRNA functional analysis and systems biology. Nucleic Acids Res 48, W244-W251, doi:10.1093/nar/gkaa467 (2020).

35 Volkmar, N. et al. The ER membrane protein complex promotes biogenesis of sterolrelated enzymes maintaining cholesterol homeostasis. J Cell Sci 132, doi:10.1242/jcs.223453 (2019).

36 Khan, T. A. et al. Neuronal defects in a human cellular model of 22q11.2 deletion syndrome. Nat Med 26, 1888-1898, doi:10.1038/s41591-020-1043-9 (2020). 
37 Langmead, B., Trapnell, C., Pop, M. \& Salzberg, S. L. Ultrafast and memory-efficient alignment of short DNA sequences to the human genome. Genome Biol 10, R25, doi:10.1186/gb-2009-10-3-r25 (2009).

38 Wideman, J. G. The ubiquitous and ancient ER membrane protein complex (EMC): tether or not? F1000Res 4, 624, doi:10.12688/f1000research.6944.2 (2015).

39 Benger, M., Kinali, M. \& Mazarakis, N. D. Autism spectrum disorder: prospects for treatment using gene therapy. Mol Autism 9, 39, doi:10.1186/s13229-018-0222-8 (2018).

40 Becker, L. A. et al. Therapeutic reduction of ataxin-2 extends lifespan and reduces pathology in TDP-43 mice. Nature 544, 367-371, doi:10.1038/nature22038 (2017).

41 DeVos, S. L. et al. Tau reduction prevents neuronal loss and reverses pathological tau deposition and seeding in mice with tauopathy. Sci Transl Med 9, doi:10.1126/scitranslmed.aag0481 (2017).

42 Kordasiewicz, H. B. et al. Sustained therapeutic reversal of Huntington's disease by transient repression of huntingtin synthesis. Neuron 74, 1031-1044, doi:10.1016/j.neuron.2012.05.009 (2012).

43 Korobeynikov, V. A., Lyashchenko, A. K., Blanco-Redondo, B., Jafar-Nejad, P. \& Shneider, N. A. Antisense oligonucleotide silencing of FUS expression as a therapeutic approach in amyotrophic lateral sclerosis. Nat Med 28, 104-116, doi:10.1038/s41591-02101615-z (2022).

44 Scoles, D. R. et al. Antisense oligonucleotide therapy for spinocerebellar ataxia type 2. Nature 544, 362-366, doi:10.1038/nature22044 (2017).

45 Sztainberg, Y. et al. Reversal of phenotypes in MECP2 duplication mice using genetic rescue or antisense oligonucleotides. Nature 528, 123-126, doi:10.1038/nature16159 (2015).

46 Tabrizi, S. J., Smith, A. V. \& Bennett, C. F. Targeting Huntingtin in Patients with Huntington's Disease. Reply. N Engl J Med 381, 1181-1182, doi:10.1056/NEJMc1910544 (2019).

47 Winkelsas, A. M. \& Fischbeck, K. H. Nucleic acid therapeutics in neurodevelopmental disease. Curr Opin Genet Dev 65, 112-116, doi:10.1016/j.gde.2020.05.022 (2020).

48 Rinaldi, C. \& Wood, M. J. A. Antisense oligonucleotides: the next frontier for treatment of neurological disorders. Nat Rev Neurol 14, 9-21, doi:10.1038/nrneurol.2017.148 (2018).

49 Shao, Y. et al. Antisense oligonucleotide therapy in a humanized mouse model of MECP2 duplication syndrome. Sci Transl Med 13, doi:10.1126/scitranslmed.aaz7785 (2021).

50 Karczewski, K. J. et al. The mutational constraint spectrum quantified from variation in 141,456 humans. Nature 581, 434-443, doi:10.1038/s41586-020-2308-7 (2020).

51 Shao, D. D. et al. A recurrent, homozygous EMC10 frameshift variant is associated with a syndrome of developmental delay with variable seizures and dysmorphic features. Genet Med 23, 1158-1162, doi:10.1038/s41436-021-01097-x (2021). 


\section{Figure Legends}

Figure 1: Altered gene expression in hiPSC-derived cortical neurons with 22q11.2 deletion. (a) Schematic diagram depicting the human chromosome 22q11.2 region. Bright grey and red horizontal bars indicate the two most common hemizygous genomic deletions found in the 22q11.2 deletion syndrome. The location of the coding genes and non-coding RNAs (miRNAs, underlined) are shown for chromosome 22q11.2. The microprocessor DGCR8 (DiGeorge Syndrome Critical Region Gene 8) and mir185 are shown in bold. (b) Cortical marker TBR1 and pan-neuronal marker TUJ1 expression in cortical neurons as detected by immunocytochemistry at day 13 of differentiation. TBR1 (red), TUJ1 (green) and DAPI (blue) expression are shown. Scale bars: $100 \mu \mathrm{m}$. (c) Volcano plot showing differential gene expression in cortical neurons at day 8 of differentiation. Significantly differentially expressed genes (FDR $<5 \%)$ are shown above red line; Q5 (Ctrl) n=3, Q6 (22q11.2) n=3. 1937/2094 genes were significantly up- and downregulated in Q6 (22q11.2) hiPSC-derived cortical neurons, respectively. 22q11.2 deletion region residing genes RANBP1 and DGCR8 as well as EMC10 are highlighted. (d-e) Gene Ontology (GO) term enrichment analysis of up- and down-regulated DEGs reveals several neuronal related components. (d) Biological Process. (e) Cellular Component. (f) qRT-PCR analysis confirming reduction of $D G C R 8(\mathrm{P}=0.0261)$ and $R A N B P 1(\mathrm{P}=0.0 .0006)$ mRNAs but not for the housekeeping control RPLPO $(\mathrm{P}=0.7155)$ gene at day 8 of differentiation in $\mathrm{Q} 6(22 \mathrm{q} 11.2)$ compared to Q5(Ctrl) lines derived cortical neurons (Q5: $n=3, Q 6: n=3)$. (g) Western blot analysis showing reduced amount of DGCR8 and RANBP1 protein in Q6 (22q11.2) line derived cortical neurons at day 8 of differentiation. Tubulin was probed as a loading control. (h) Consistent upregulation of EMC10 mRNA in Q6 (22q11.2) line derived cortical neurons as assayed by qRT$P C R$ at day $8(P=0.031))$, day $20(P=0.0478)$ and day $34(P=0.0358)$ of differentiation $(Q 5: n=3$, Q6: n=3). (i) Western blot analysis showing upregulated EMC10 protein levels in Q6 (22q11.2) line derived cortical neurons at day 8 of differentiation. Tubulin was probed as a loading control. (j) qRT-PCR analysis confirming upregulation of EMC10 (P=0.0338) mRNA in Q27 (22q11.2) 
compared to Q20 (Ctrl) lines derived cortical neurons at day 8 of differentiation (QR20: $n=7$, QR27: n=5). (k) Immunofluorescence images of NGN2 generated cells. Representative images of NGN2-iNs at DIV21 from Q5 (Ctrl) and Q6 (22q11.2) hiPSC lines identified via EGFP fluorescence and immunostained for neuronal dendrite marker MAP2, the cortical marker TBR1 and the nuclear marker DAPI. Scale bars=100 $\mu \mathrm{m}$. (I-n) qRT-PCR assay of EMC10 mRNA expression level in NGN2-iNs at DIV21. (I) Upregulation of EMC10 mRNA in Q6 (22q11.2) line derived neurons compared to the healthy control line $Q 5$ at day $21(P=0.0222)$. Q5 (Ctrl) n=4, Q6 (22q11.2) n=4. (m) Upregulation of EMC10 mRNA in Q1 (22q11.2) patient line compared to healthy control line Q2 (P=0.0441). Q2 (Ctrl) n=5 and Q1 (22q11.2) n=7. (n) Upregulation of EMC10 mRNA in QR27 (22q11.2) patient line compared to healthy control line QR20 ( $P=0.0414)$. QR20 (Ctrl) n=5 and QR27 (22q11.2) n=5. Data are shown as mean \pm SEM, unpaired two-tailed t-test, ${ }^{*} \mathrm{P}<0.05,{ }^{* *} \mathrm{P}<0.01$.

Figure 2: Altered miRNA expression in hiPSC-derived cortical neurons with 22q11.2 deletion (a) Volcano plot showing differentially expressed miRNAs (DEmiRs) in cortical neurons at day 8 of differentiation. Significant DEmiRs ( $P$ value $<5 \%)$ are shown above red line; Q5 (Ctrl) n=3, Q6 (22q11.2) n=3. 153/133 miRNAs were significantly up- and downregulated in Q6 (22q11.2) hiPSC-derived cortical neurons, respectively. 22q11.2 deletion region residing miRNAs miR-185, miR-1286 and miR-1306 are highlighted. (b-c) Gene Ontology (GO) term enrichment analysis of up- and down-regulated DEmiRs highlighted cell cycle and cell division relevant terms. (b) Biological Process. (c) Cellular Component. (d) Intersection of predicted miRNA targets and gene expression analysis. Venn diagram highlighting up-regulated DEGs (from bulk RNAseq) that are predicted targets of down-regulated miRNAs (774). (e-f) Gene Ontology (GO) term enrichment analysis of predicted targets reveals several neuronal related components for the upregulated genes. (e) Biological Process. (f) Cellular Component. (g) Precursor miRNA expression level of miR-185 $(P=0.0038)$ and miR-485 $(P=0.0622)$, predicted to target EMC10, are 
downregulated in Q6 (22q11.2) cortical neurons as assayed by qRT-PCR (Q5: n=3, Q6: n=3). (h) qRT-PCR quantification shows reduced expression levels of EMC10 mRNA in Q5 (Ctrl) line derived cortical neurons at day 10 of differentiation transfected with miR-185 $(P=0.0481)$ or miR$485(P=0.0334)$ mimics at day 8 of differentiation. Expression levels in miR-185 or miR-485 mimic-treated neurons were normalized to expression levels under scramble mimic controls treatment ( $n=3$, each treatment). (i) qRT-PCR quantification shows reduced expression levels of EMC10 mRNA in Q6 line-derived cortical neurons transfected with miR-185 $(\mathrm{P}<0.01)$ or miR-485 $(P=0.0027)$ or a combination of both miRNA mimics $(P=0.0134)$. Expression levels in miR-185, miR-485 or the combination of both mimic-treated neurons were normalized to expression levels under scramble mimic controls treatment. Ctrl mimic $n=5$, miR-185 mimic $n=5$, miR-485 mimic $n=4$ and miR185+miR485 mimics $n=3$. (j) qRT-PCR quantification shows increased expression levels of EMC10 mRNA in Q5 line derived cortical neurons transfected with miRNA inhibitors miR$185(\mathrm{P}<0.01)$ or miR-485 $(\mathrm{P}=0.0236)$ at day 8 of differentiation. Expression levels in miR-185 or miR-485 inhibitor-treated neurons were normalized to expression levels under scramble miRNA inhibitor controls treatment $(n=3$, each treatment). Data are shown as mean $\pm S E M$, unpaired twotailed t-test, ${ }^{*} \mathrm{P}<0.05,{ }^{*} \mathrm{P}<0.01$.

Figure 3: Reduction of EMC10 levels restores defects in neurite outgrowth and calcium signaling in neurons from 22q11.2 deletion carriers. (a) qRT-PCR assay in hiPSC lines. EMC10 expression is reduced or abolished in $\mathrm{Q6} / \mathrm{EMC} 10^{\mathrm{HET}}$ line $(\mathrm{P}=0.0002)$ and $\mathrm{Q6} / \mathrm{EMC} 10^{\mathrm{HOM}}$ line $(P=0.0003)$, respectively, when compared to $Q 5(C t r l)$ line. Note that EMC10 is not upregulated in Q6 hiPSCs. Q5 (Ctrl) $n=5, Q 6$ (22q11.2) $n=5, Q 6 / E M C 10^{\text {HET }} \mathrm{n}=6$ and Q6/EMC10 ${ }^{\mathrm{HOM}} \mathrm{n}=4$. (b) Representative images of NGN2-iNs at DIV21 from Q6/EMC10 ${ }^{\mathrm{HET}}$ and Q6/EMC10 $10^{\text {HOM }}$ hiPSC lines identified via EGFP fluorescence and immunostained for neuronal dendrite marker MAP2, the cortical marker TBR1 and the nuclear marker DAPI. Scale bars=50 $\mu \mathrm{m}$. (c) qRT-PCR assay of EMC10 mRNA expression level in NGN2-iNs at DIV21. EMC10 
expression is normalized to near WT levels level in Q6/EMC10 ${ }^{\mathrm{HET}}$ line $(\mathrm{P}=0.166)$ and abolished in Q6/EMC10 ${ }^{\text {HOM }}$ line $(P<0.0001)$. Q5 (Ctrl) n=4, Q6 (22q11.2/SCZ) $n=4, Q 6 / E M C 10^{\text {HET }} n=5$ and Q6/EMC10 ${ }^{\text {HOM }} \mathrm{n}=7$. (d-h) Neuronal morphology analysis in Q5, Q6 (22q11.2), Q6/EMC10 ${ }^{\text {HET }}$ and Q6/EMC10 $0^{\mathrm{HOM}}$ neurons. (d) Representative images of traced neurons. (e) Total neuronal length is reduced in $\mathrm{Q} 6$ line $(P=0.0044)$ and restored in $Q 6 / \mathrm{EMC}^{\mathrm{HET}}(\mathrm{P}=0.0253)$ and $\mathrm{Q6} / \mathrm{EMC} 10^{\mathrm{HOM}}$ line $(P=0.0001)$. (f) Reduction in number of branch points/cell in $Q 6(P=0.0195)$ is restored in the Q6/EMC10 ${ }^{\mathrm{HET}}(\mathrm{P}=0.0134)$ and $\mathrm{Q6} / \mathrm{EMC10}{ }^{\mathrm{HOM}}$ lines $(\mathrm{P}<0.0001)$. (g) Reduction in the total number of dendrites/cells in Q6 $(P=0.0202)$ is reversed in the $Q 6 / E M C 10^{H E T} \quad(P=0.0166)$ and Q6/EMC10 ${ }^{\text {HOM }}$ lines $(P=0.0005)(h)$ The number of primary dendrites per cell is unchanged. Q5 (Ctrl) n=14, Q6 (22q11.2) n=16, Q6/EMC10 ${ }^{\text {HET }} n=19$ and Q6/EMC10 ${ }^{\text {HOM }} n=16$ neuronal cells. (iI) Defects in cytoplasmic calcium signaling in Q6 (22q11.2) neurons are reversed in Q6/EMC10 ${ }^{\mathrm{HET}}$ and Q6/EMC10 ${ }^{\mathrm{HOM}}$ lines. (i) Changes in Fluo4-AM fluorescence signal intensity in response to $75 \mathrm{mM} \mathrm{KCl}$ in Q5 (Ctrl) and Q6 (22q11.2) hiPSC-derived neurons at DIV37 (KS D=0.4865, $\mathrm{P}=0.003)$. (j) Quantification of $\mathrm{KCl}$-induced Fluo4 intensity peak amplitude $(\Delta \mathrm{F})$ shows a reduction in Q6 line ( $P<0.0001)$. Q5 (Ctrl) n=21, Q6 (22q11.2) n=24 neuronal cells. (k) Changes in Fluo4AM fluorescence signal intensity in response to $75 \mathrm{mM} \mathrm{KCl}$ in Q5 (Ctrl), Q6 (22q11.2). Q6/EMC10 ${ }^{\mathrm{HET}}$ and Q6/EMC10 ${ }^{\mathrm{HOM}}$ hiPSC-derived neurons at DIV38. Q5 vs. Q6 (KS D=0.5405, $\mathrm{P}<0.0001$ ), Q6 vs. Q6 EMC10 ${ }^{\mathrm{HET}}$ (KS D=0.5556, $\mathrm{P}<0.0001$ ) and Q6 vs. Q6 EMC10 ${ }^{\mathrm{HOM}}$ (KS $\mathrm{D}=0.5676, \mathrm{P}<0.0001)$. (I) Quantification of $\mathrm{KCl}$-induced Fluo4 intensity peak amplitude $(\Delta \mathrm{F})$ demonstrates a reduction in $Q 6$ line $(P<0.0001)$ that is reversed in $Q 6 / E M C 10^{H E T}(P<0.0001)$ and Q6/EMC10 ${ }^{\text {HOM }}$ lines ( $\left.P<0.0001\right)$. Q5 (Ctrl) n=70, Q6 (22q11.2/SCZ) n=37, Q6/EMC10 ${ }^{\text {HET }} n=82$, Q6/EMC $10^{\text {HOM }} \mathrm{n}=97$ neuronal cells. Data are shown as mean \pm SEM, unpaired two-tailed t-test or Kolmogorov-Smirnov test as indicated, ${ }^{*} \mathrm{P}<0.05,{ }^{* *} \mathrm{P}<0.01,{ }^{* * *} \mathrm{P}<0.001,{ }^{* * *} \mathrm{P}<0.0001$.

Figure 4: ASO-mediated suppression of murine Emc10 in vitro and in vivo. (a) Emc10 mRNA levels in $4 \mathrm{~T} 1$ cells following $7 \mu \mathrm{M}$ treatment with Emc10 ASOs, data normalized to untransfected 
controls (UTC), and lead Emc10 ASO1 noted in red. (b) Dose dependent reductions in Emc10 mRNA levels in $4 \mathrm{~T} 1$ cells following treatment with lead Emc10 ASO1, but not with a negative

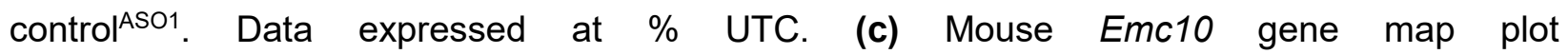
(ENSMUST00000118808) showing the Emc10 ASO1 target site. (d) qRT-PCR analysis shows bilateral reduction of Emc10 mRNA expression levels in Emc10 ${ }^{A S O 1}$ compared to the $\mathrm{Ctrl}{ }^{\mathrm{ASO} 1}$ treated WT mice in the left (unpaired two-tailed t-test, $\mathrm{P}=0.0285 ; \mathrm{Ctr}{ }^{\mathrm{ASO}}{ }^{1}$ treated-WT male mice: $\mathrm{n}=8, E m c 10^{\mathrm{ASO} 1}$ treated-WT male mice: $\mathrm{n}=8$ ) and the right hippocampus (unpaired two tailed $\mathrm{t}$ test, $\mathrm{P}=0.0087 ; \mathrm{Ctr} \mathrm{ASO}^{\mathrm{ASO}}$ treated-WT mice: $\mathrm{n}=7$, and Emc10 $0^{\mathrm{ASO} 1}$ treated-WT mice: $\left.\mathrm{n}=8\right)$. (e) Western blot analysis shows bilateral reduction of Emc10 protein expression in the left $(\mathrm{P}=$ 0.0321; unpaired two-tailed t-test; $\mathrm{Ctr}^{\mathrm{ASO} 1}$ treated-WT male mice: $\mathrm{n}=3$, Emc10 ${ }^{\mathrm{ASO} 1}$ treated-WT male mice: $n=4)$ and right hippocampus $(P=0.0448$; unpaired two-tailed t-test; $C$ tr|ASO1 treatedWT male mice: $\mathrm{n}=3$, Emc10 ${ }^{\mathrm{ASO} 1}$ treated-WT male mice: $\left.\mathrm{n}=3\right)$. (f) mRNA expression analysis of astroglial (Gfap, left panel; $\mathrm{P}=0.177$; one-way ANOVA) and microglial (Aif1, right panel; $\mathrm{P}=$ 0.1527; one-way ANOVA) activation revealed no significant differences across groups. Untreated male mice; $\mathrm{n}=4, \mathrm{Ctr}{ }^{\mathrm{ASO} 1}$ treated-WT male mice: $\mathrm{n}=4, E m c 10^{\mathrm{ASO} 1}$ treated-WT male mice: $\mathrm{n}=4 . \mathbf{( g )}$ Mouse brains collected three weeks post ICV injection were stained with an ASO antibody (green), counterstained with neuronal marker NeuN (red) and nuclear stain Hoechst (blue). A robust and uniform ASO diffusion (top panel) is observed in the hippocampus. No signal is detected in saline injected mice (middle panel). Overlap with NeuN (yellow, top-right panel) confirms presence in neuronal cells. Accumulation in glial cells, specifically GFAP labeled astrocytes is also observed (bottom-right panel and inset; ASO in green, GFAP in red, and Hoechst in blue). Images are taken with 4x, 20x and 40x objectives. (h): qRT-PCR analysis shows Emc10 ${ }^{\text {ASO1 }}$ meditated normalization of Emc10 mRNA levels in the hippocampus of $D f(16) A^{+/-}$mice (left panel). Significant upregulation of Emc10 mRNA expression levels is seen in $\mathrm{Ctr} \mathrm{ASO}^{\mathrm{AS}}$ treated$D f(16) A^{+/}$compared to WT mice [one-way ANOVA, $F(2,29)=11.65, P<0.001$; post hoc Tukey, $\mathrm{P}=0.001]$. Following ASO treatment, Emc10 expression is normalized to WT levels in Emc10 ASO1 
treated-Df(16) $A^{+/}$compared to $\mathrm{Ctrl}^{\mathrm{ASO}}$ treated-Df(16) $A^{+/-}$mice (post hoc Tukey, $\mathrm{P}=<0.001$ ). Ctrl|ASO1-treated WT mice: $\mathrm{n}=9$ (5 males, 4 females), $\mathrm{Ctrl}^{\mathrm{ASO}}{ }^{\mathrm{AS}}$-treated $\mathrm{Df}(16) \mathrm{A}^{+/}$mice: $\mathrm{n}=11(5$ males, 6 females), and Emc10 ASO1-treated $D f(16) A^{+/}$mice: $n=12$ (7 males, 5 females). qRT-PCR analysis shows a significant up-regulation of Emc10 mRNA expression levels in the PFC of $\mathrm{Ctrl}^{\mathrm{ASO} 1}$ treated-Df(16)A $\mathrm{A}^{+/}$(right panel) compared to WT mice [one-way ANOVA, F $(2,16)=4.253$, $\mathrm{P}=0.0330$; post hoc Tukey, $\mathrm{P}=0.0385]$. ASO injection does not normalize Emc10 expression levels in PFC of $\mathrm{Df}(16) \mathrm{A}^{+/}$mice injected with Emc10 ${ }^{\mathrm{ASO} 1}$ (Post hoc Tukey vs Ctrl ${ }^{\mathrm{ASO} 1}$ treated $D f(16) A^{+/}$mice, $\left.\mathrm{P}=0.7642\right) . \mathrm{Ctrl}{ }^{\mathrm{ASO} 1}$ treated-WT male mice: $\mathrm{n}=6, \mathrm{Ctrl} \mathrm{ASO}^{\mathrm{A}}$ treated-Df(16) $A^{+/-}$male mice: $\mathrm{n}=5$, and Emc10 $10^{\mathrm{ASO} 1}$ treated-Df(16) $A^{+/}$male mice: $\mathrm{n}=8$. (i) Volcano plots showing upregulation of Emc10 expression in the $\mathrm{Ctr}^{\mathrm{ASO} 1}$ treated-Df(16) $A^{+/}$compared to the $\mathrm{Ctr}{ }^{\mathrm{ASO}}$-treated WT mice (left panel and inset) but not in the Emc10 ${ }^{\mathrm{ASO} 1}$ treated-Df(16) $A^{+-}$compared to the Emc10 ${ }^{\mathrm{ASO} 1}$-treated WT mice (right panel and inset). The expected down-regulation of genes included in the $D f(16) A^{+/-}$deletion (blue) and upregulation of non-coding RNAs (ncRNAs, red) is also evident in both panels. Downregulated genes from the 22q11.2 locus (Dgcr8, Ranbp1 and Tango2) as well as the upregulated ncRNA (miRNA-containing) gene Mirg, are highlighted. Ctrl|ASO1 treated-Df(16)A $A^{+/}$males: $n=5, E m c 10^{A S O 1}$ treated-Df(16) $A^{+/-}$males: $n=4, C$ tr| $\left.\right|^{\mathrm{ASO} 1}$ treated WT males: $n=4$, and Emc10 ${ }^{\text {ASO1 }}$ treated-WT males: $n=4$. Data are presented as mean \pm SEM. *P $<0.05 ;{ }^{* *} \mathrm{P}<0.01 ;{ }^{* *} \mathrm{P}<0.001$

Figure 5: ASO mediated rescue of SM deficit in $D f(16) A^{+/-}$mice. (a) $\left.\mathrm{Ctr}\right|^{A S O 1}$-treated $D f(16) A^{+/-}$ mice show a robust SM deficit compared to $\mathrm{Ctrl}^{\mathrm{ASO}}$-treated WT mice as indicated by the significant difference in trial 2 interaction time upon reintroduction of a familiar juvenile mouse [three-way ANOVA for Trial X Genotype $X$ Treatment Interaction matching by trial: $F(1,38)=$ 9.393 $\mathrm{P}=0.0040$; post hoc Tukey, $\mathrm{P}=0.0012$ ]. A reduction in trial 2 interaction time indicates rescue of the SM deficit in Emc10 ${ }^{\mathrm{ASO} 1}$-treated $D f(16) A^{+/-}$compared to $\mathrm{Ctrl}{ }^{\mathrm{ASO} 1}$-treated $D f(16) A^{+/-}$ mice (post hoc Tukey, $P=0.0114$ ). (b) A negative difference score (trial 1- trial 2) confirms the SM 
deficit in $\mathrm{Ctrl}^{\mathrm{ASO}}$-treated adult $\mathrm{Df}(16) \mathrm{A}^{+/-}$mice compared to WT littermates [two-way ANOVA for Genotype X Treatment interaction: $F(1,38)=9.369, P=0.0040$; post hoc Tukey, $P=0.0002]$. Increase in the difference score of $D f(16) A^{+-}$mice in the Emc10ASO1- vs CtrlASO1_ treated group demonstrates SM rescue (post hoc Tukey, $\mathrm{P}=0.0004)$. [CtrlAso1-treated WT mice: $\mathrm{n}=9$ (5 males, 4 females), $\mathrm{Ctrl}^{\mathrm{ASO} 1}$-treated $D f(16) A^{+/}$mice: $\mathrm{n}=11$ (5 males, 6 females), Emc10 ${ }^{\mathrm{ASO} 1}$ treated WT

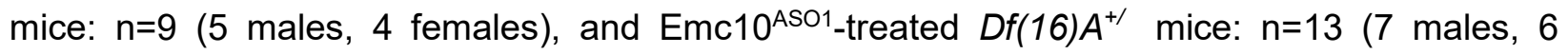
females)]. (c) No significant difference in SM trials is observed across groups upon reintroduction of a novel juvenile mouse indicating SM rescue is not due to task fatigue [three-way ANOVA for Trial X Genotype X Treatment matching by trial; $F(1,30)=0.3001, P=0.5878$ ]. (d) No significant changes across groups in SM difference score upon reintroduction of a novel juvenile mouse [two-way ANOVA for Genotype X Treatment interaction; $F(1,30)=0.3001 P=0.5878]^{\text {. Ctrl }}{ }^{\text {ASO1_ }}$ treated WT: $\mathrm{n}=8$ (4 males, 4 females), Emc10 ${ }^{\text {ASO1 }}$ treated WT: $\mathrm{n}=10$ (4 males, 6 females), $\mathrm{n}=8$ Ctrl ${ }^{\mathrm{ASO} 1}$-treated $\mathrm{Df}(16) A^{+/}$(3 males, 5 females) and Emc10 ${ }^{\mathrm{ASO} 1}$-treated $D f(16) A^{+/}: \mathrm{n}=8$ (4 males, 4 females). (e) In a contextual fear memory assay, minimal freezing is observed on day 1 (left) with no significant changes across groups [two-way ANOVA for Genotype X Treatment interaction: F $(1,52)=1.003, P=0.3211]$. In the $C \operatorname{trl}^{\mathrm{ASO}}$-treated group, $D f(16) A^{+/}$mice show the expected contextual fear memory deficit compared to WT mice (right panel) [one-way ANOVA, F $(3,52)=$ 3.524 $\mathrm{P}=0.0212$; post hoc Tukey, 0.0384]. Emc10ASO1 treatment was not sufficient to fully rescue the learning deficit in $D f(16) A^{+/}$mice compared to Ctrl ASO1-treated WT levels (post hoc Tukey, $\mathrm{P}=0.1045)$. However, there is increased freezing in $D f(16) A^{+/-}$mice injected with Emc10 ${ }^{\text {ASO1_ }}$ versus $\mathrm{Ctr}^{\mathrm{ASO} 1}$-treated group, which results in a non-significant difference in freezing between

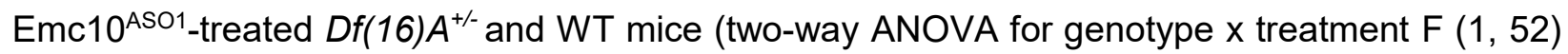
$=0.8524, P=P=0.3601$; post hoc Tukey, $P=0.4676$ ) indicating a partial rescue of the contextual fear memory deficit. CtrlASO1-treated WT: $n=13$ (9 males, 4 females), Emc10 ${ }^{\text {ASO1 }}$ treated WT mice: $\mathrm{n}=14$ (10 males, 4 females), Ctrl|ASO1-treated $D f(16) A^{+/}$mice: $\mathrm{n}=15$ (11 males, 4 females and 
Emc10 ${ }^{\text {ASO1_treated }} \mathrm{Df}(16) A^{+/}$mice: $\mathrm{n}=14$ (10 males, 4 females). Data are presented as mean \pm SEM. * $\mathrm{P}<0.05 ;{ }^{* *} \mathrm{P}<0.01 ;{ }^{* * *} \mathrm{P}<0.001$.

Figure 6: Rescue of SM deficit in $D f(16) A^{+/}$mice using an independent ASO: (a) Mouse Emc10 gene map plot (ENSMUST00000118808) showing the Emc10 ASO2 target site. (b) Mouse brains collected three weeks post ICV injection were stained with an ASO antibody (green) and counterstained with neuronal marker NeuN (red) and nuclear stain Hoechst (blue). A robust and uniform ASO diffusion is observed in the hippocampus (top panel) and PFC (middle panel). Overlap with NeuN confirms its presence in neuronal cells. Accumulation in glial cells, specifically GFAP labeled astrocytes is also observed. Images are taken with $10 x$ and 20x objectives. (c) qRT-PCR analysis shows significant upregulation of Emc10 mRNA expression levels in the hippocampus of $\mathrm{Ctr}{ }^{\mathrm{ASO}}$ treated-Df(16) $A^{+/}$compared to WT mice [one-way ANOVA, F $(2,19)=$ 20.92, $\mathrm{P}<0.0001$; post hoc Tukey, $\mathrm{P}=0.0018$ ]. Following ASO treatment, Emc10 expression is normalized to WT levels in Emc10 ${ }^{\mathrm{ASO} 2}$ treated-Df(16) $A^{+/-}$compared to $\mathrm{Ctr} \mathrm{ASO}^{\mathrm{ASO}}$ treated-Df(16) $A^{+/-}$ mice (post hoc Tukey, $P=<0.0001$ ). (d) qRT-PCR analysis shows a significant up-regulation of Emc10 mRNA expression levels in the PFC of $\mathrm{Ctrl}^{\mathrm{ASO} 2}$ treated-Df(16)A $A^{+/}$compared to WT mice [one-way ANOVA, $\mathrm{F}(2,19)=40.75, \mathrm{P}=<0.0001$; post hoc Tukey, $\mathrm{P}=<0.0001$ ]. Following ASO treatment, Emc10 expression is normalized to WT levels in the Emc10 AsO2 treated-Df(16)A ${ }^{+/}$ compared to $\mathrm{Ctrl}^{\mathrm{ASO} 2}$ treated-Df(16) $A^{+/}$mice (Post hoc Tukey, $\left.\mathrm{P}=<0.0001\right)$. Ctrl ${ }^{\mathrm{ASO} 2}$-treated WT male mice: $\mathrm{n}=6, \mathrm{Ctrl}^{\mathrm{ASO} 2}$-treated $D f(16) A^{+/}$male mice: $\mathrm{n}=8$, and Emc10 ${ }^{\mathrm{ASO}}$-treated $D f(16) A^{+/}$mice: $\mathrm{n}=8$. (e) $q R T-P C R$ analysis shows a significant up-regulation of Emc10 mRNA expression levels in Somatosensory Cortex (SSC) of $\mathrm{Ctr}^{\mathrm{ASO} 2}$ treated-Df(16) $A^{+-}$compared to WT mice [one-way ANOVA, $F(2,18)=21.53, P=<0.0001$; post hoc Tukey, $\mathrm{P}=<0.0026$ ]. Following ASO treatment, Emc10 expression is normalized to WT levels in Emc10 $10^{\mathrm{ASO} 2}$ treated-Df(16)A $A^{+/}$compared to Ctrl ${ }^{A S O 2}$ treated-Df(16) $A^{+/-}$mice (Post hoc Tukey, $\left.\mathrm{P}=<0.0001\right)$. Ctrl ${ }^{\mathrm{ASO} 2}$-treated WT male mice: $\mathrm{n}=6,\left.\mathrm{Ctr}\right|^{\mathrm{ASO} 2}$-treated $D f(16) A^{+/}$male mice: $\mathrm{n}=7$, and Emc10 ${ }^{\mathrm{ASO} 1}$-treated $D f(16) A^{+/}$mice: $\mathrm{n}=8$. (f) 


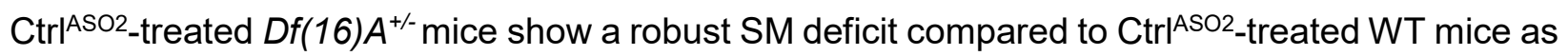
indicated by the significant difference in trial 2 interaction time upon reintroduction of a familiar juvenile mouse [three-way ANOVA for Trial X Genotype X Treatment Interaction matching by trial: $F(1,26)=35.74 P<0.0001 ;$ post hoc Tukey, $P=0.0004]$. A reduction in trial 2 interaction time indicates rescue of the $\mathrm{SM}$ deficit in Emc10 ${ }^{\mathrm{ASO} 2}$-treated $\mathrm{Df}(16) \mathrm{A}^{+/-}$compared to $\mathrm{Ctr}^{\mathrm{ASO} 2}$-treated $D f(16) A^{+-}$mice (post hoc Tukey, $\left.\mathrm{P}=0.0255\right)$. [Ctrl|${ }^{\mathrm{ASO}}$-treated WT male mice: $\mathrm{n}=6,\left.\mathrm{Ctr}\right|^{\mathrm{ASO}}$-treated $D f(16) A^{+/}$mice: $\mathrm{n}=8$, Emc10 ${ }^{\mathrm{ASO} 2}$ treated WT mice: $\mathrm{n}=8$, and Emc10ASO2-treated $D f(16) A^{+/}$mice: $\mathrm{n}=8$. (g) A negative difference score (trial 1- trial 2) confirms the SM deficit in $\mathrm{Ctrl}^{\mathrm{ASO}}$-treated adult $D f(16) A^{+/}$mice compared to WT littermates [two-way ANOVA for Genotype $X$ Treatment interaction $F(1,26)=35.74, P<0.0001$; post hoc Tukey, $\mathrm{P}<0.0001$. Increase in the difference score of $D f(16) A^{+/-}$mice in the Emc10 $\mathrm{ASO}_{-}$vs $\mathrm{Ctrl}^{\mathrm{ASO} 2}$ - treated group demonstrates SM rescue (post hoc Tukey, $P<0.0001$ ). Data are presented as mean \pm SEM. ${ }^{*} P<0.05 ;{ }^{* *} P<0.01$; ${ }^{* *} P<$ $0.001 ;{ }^{* * * *} \mathrm{P}<0.0001$ 


\section{Methods}

Mice: We used $D f(16) A^{+/}$mice ${ }^{5}$ in C57BL/6J background. $D f(16) A^{+/}$male mice were crossed to C57BL/6J female mice to obtain either $D f(16) A^{+/-}$or WT littermates. Mice of both sexes and genotypes (mutant and WT littermates) were used for behavioral testing. Separate cohorts of mice were used for Social Memory and Fear Conditioning assays. In general, mice were group housed under a 12-h light/12-h dark cycle with controlled room temperature and humidity. Food and water were provided ad libitum. All behavioral experiments were performed on adult male and female mice during the light cycle. All animal procedures were carried out in accordance with and approved by the Columbia University Institutional Animal Care and Use Committee.

\section{Cell line donors}

Q6 and Q5 lines: The Q6 line donor is a 20-year-old female patient with a history of developmental delay and an overall Full-Scale IQ in the low 80 s. She was clinically diagnosed with 22q11.2DS by FISH testing. Her psychotic symptoms, including disorganized behavior and command auditory hallucinations, started when she was 17 years old. During the first break episode, due to the severity of her psychotic symptoms, the patient was hospitalized and was diagnosed with schizophrenia. The patient also developed depressive symptoms, including frequent suicidal ideation. One year after her schizophrenia diagnosis, in addition to her severe psychotic symptoms, the patient was also diagnosed to be in a catatonic state. The patient has remained severely psychotic since the onset of these symptoms at age 17 and has been on multiple antipsychotics without experiencing any clinically meaningful benefit. Regarding her treatment history includes various first-line antipsychotics (including olanzapine, stelazine, aripiprazole, haloperidol, risperidone and clozapine); several antidepressants (sertraline and fluoxetine); a mood stabilizer (lithium) and benzodiazepines (e.g., lorazepam). None of these medications reportedly led to any clinically significant improvement in either the psychotic or the depressive symptoms. The patient has also undergone 2 rounds of electroconvulsive treatment (ECT), but 
with only short-lived improvement. The Q5 line donor is the probands monozygotic twin sister, who does not carry a 22q11.2 deletion and her psychiatric evaluation ruled out any history of psychiatric symptoms. Sibling are of Caucasian Western European descent.

Q1 and Q2 lines: The Q1 and Q2 line were previously described (as DEL3 and WT3) ${ }^{52}$. The donor of the Q1 line is a male diagnosed with schizoaffective disorder and 22q11.2DS while the donor of the Q2 line (male sibling) was free from any psychiatric symptoms (Supplementary Table S1). Siblings are of Caucasian Western European descent.

QR20 and QR27 lines: QR20 (MH0159020) and QR27 (MH0159027) lines were obtained from the NIMH Repository and Genomics Resource (http://www.nimhstemcells.org/) ${ }^{53}$. The donor of the QR27 line, 31-year-old male was diagnosed with schizoaffective disorder and 22q11.2DS ${ }^{53}$ while the donor of the QR20 line (58 year old male) was free from any psychiatric symptoms (Supplementary Table S1). Both are of Caucasian descent.

hiPSC generation and characterization. Q5 and Q6 iPSC lines were generated at the Columbia Stem Cell Core via non-integrating Sendai virus-based reprogramming ${ }^{54}$ of monocytes from a donor with 22q11.2DS and SCZ and a healthy sibling control. The Q1 and Q2 lines were generated at the Columbia Stem Cell Core and characterized as described earlier ${ }^{52}$. QR20 and QR27 lines were obtained from the NIMH Repository and Genomics Resource (http://www.nimhstemcells.org//53. Karyotyping was performed on twenty G-banded metaphase cells at $450-500$ band resolution as previously described ${ }^{55}$ to ensure the absence of chromosomal abnormalities in all patient and control derived cell lines (Fig. S1a, Fig. S5b). We confirmed the genotypes of Q6 patient- and Q5 control-derived hiPSCs using a Multiplex Ligation-dependent Probe Amplification (MLPA) assay to detect copy number changes (Fig. S1b-c). To confirm stemness of hiPSC lines, we performed qRT-PCR for markers NANOG and OCT4/POU5F1 (Fig. S1d). 
Genome editing of Q6(22q11.2) hiPCS line. We generated derivatives of the Q6 (22q11.2) hiPSC line carrying either heterozygous (Q6/EMC10 $\left.{ }^{\mathrm{HET}}\right)$ or homozygous (Q6/EMC10 $\left.{ }^{\mathrm{HOM}}\right)$ EMC10 LoF mutations (performed at Applied Stemcell Inc., Milpitas, CA, USA) using standard CRISPR/Cas9 genome editing approaches. The genomic gRNA target sequences were EMC10g1: ACAGTGCCAACTTCCGGAAG (PAM suffix: CGG) and EMC10-g2: GGGACAAGGTACCATCCTGC (PAM suffix: TGG). Mutations in EMC10 were confirmed by NGS and no off-target candidates were predicted in both lines using COSMID tool (https://crispr.bme.gatech.edu) ${ }^{56}$. Karyotyping confirmed normal chromosome complement in both modified lines (Fig. S5b). qRT-PCR assays were performed in both lines for the hiPSC markers NANOG and OCT4/POU5F1 to confirm stemness as well as for genes located within 22q11.2 locus to confirm the deletion (Fig. S5c-f). Western blot analysis was performed to confirm reduction or elimination of EMC10 protein levels in the EMC10 LoF mutant lines (Fig. S5g) .

Culture and neuronal induction of hiPSC lines: hiPSC lines were maintained in mTeSR Plus medium (catalog\#05825, Stemcell Technologies, Vancouver, Canada) on Matrigel (catalog\#354277, Corning, Corning, NY, USA) coated tissue culture plate. Cells were fed on any other day and passaged weekly using RelesR (catalog\#05872, Stemcell Technologies, Vancouver, Canada) dissociation reagent in accordance to their manual. Disassociated cells were pre-plated as reported earlier ${ }^{57}$ at a density of 200,000 cells $/ \mathrm{cm}^{2}$ supplemented with $10 \mu \mathrm{M} \mathrm{Y}$ 27632 (catalog\# 1254, Tocris Bioscience, Bristol, United Kingdom) on Matrigel-coated plates and differentiation started when confluent. Differentiation of hiPSC was performed as indicated below: (i) hiPSC differentiation into cortical neurons, via a combination of small molecule inhibitors, was performed as described with few modifications ${ }^{20}$. In brief, inhibitors used in LSB+X/P/S/D induction included LDN193189 (250 nM; catalog\#04-0074, Stemgent, REPROCELL USA Inc., Beltsville, MD, USA), SB431542 (10 $\mu \mathrm{M}$; catalog\#1614, Tocris Bioscience, Bristol, United Kingdom), XAV939 (5 $\mu \mathrm{M}$; catalog\#3748, Tocris Bioscience, Bristol, United Kingdom), 


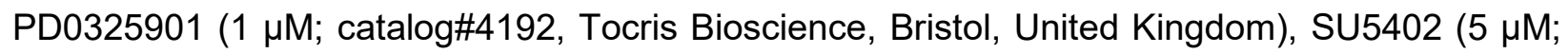
catalog\#1645-05, BioVision Inc., Milpitas, CA, USA), DAPT (10 $\mu \mathrm{M}$; catalog\#2634, Tocris Bioscience, Bristol, United Kingdom). Until day 4 of differentiation, TeSR E6 medium (catalog\#05946, Stemcell Technologies, Vancouver, Canada) was added in 1/3 increments every other day. Then, neurobasal (NB) plus medium supplemented with N2 (catalog\#17502-048, Gibco, Life Technologies, Grand Island, NY, USA) and B27 plus supplement (catalog\#A3582801, Gibco, Life Technologies, Grand Island, NY, USA) was added in 1/3 increments every other day from day 4, until reaching 100\% neurobasal plus/B27 plus L-glutamine (catalog\#35050-061, Gibco, Life Technologies, Grand Island, NY, USA) containing medium supplemented with BDNF (20 ng/ml; catalog\#248-BDB, R\&D Systems, Minneapolis, MN, USA), cAMP (0.5 mM; catalog\#A92902, Sigma-Aldrich, St. Louis, MO, USA) and ascorbic acid (0.2 mM; Sigma-Aldrich, St. Louis, MO, USA) (BCA) at day 8 as described ${ }^{20}$. For long-term culture, cells were passaged on day 8 of differentiation by Accutase (catalog\#AT-104, Innovative Cell Technologies Inc., San Diego, CA, USA) dissociation for $6 \mathrm{~min}$ at $37^{\circ} \mathrm{C}$. Cells were replated at $200,000 \mathrm{cells} / \mathrm{cm}^{2}$ onto Matrigel coated culture dishes. NB plus/B27 plus and BCA medium were used for passaging and long-term culture. Culture medium was changed every 3-4 days.

(ii) hiPSC differentiation into neurons via NGN2 overexpression was performed as described previously with few modifications ${ }^{21,23}$. In brief, differentiation of hiPSC into neurons (iNs) was conducted by using the lentiviral infection of NGN2 and the reverse tetracycline transactivator rtTa into hiPSCs, followed by selection on puromycin. The lentiviral particles were commercially produced by VectorBuilder (Chicago, IL, USA) using the established and published plasmids pLenti-FUW-M2rtTA (FUW-M2rtTA deposited by Rudolf Jaenisch, Addgene plasmid \#20342; http://n2t.net/addgene:20342; RRID:Addgene_20342, ${ }^{58}$ ), pLenti-TetO-hNGN2-eGFP-puro (pLVTetO-hNGN2-eGFP-Puro deposited by Kristen Brennand, Addgene, plasmid\#79823; http://n2t.net/addgene:79823; RRID:Addgene_79823, ${ }^{23}$ ) and for calcium imaging, the pLentiTetO-hNGN2-puro (pLV-TetO-hNGN2-Neo deposited by Kristen Brennand, Addgene plasmid \# 
99378 ; http://n2t.net/addgene:99378 ; RRID:Addgene_99378, $\left.{ }^{59}\right)$. Around 200k cells per well (24well format) were plated on Matrigel coated wells/coverslips in mTeSR plus media supplemented with $10 \mu \mathrm{M}$ Y-27632. On day 0 , lentiviruses were added in fresh basic media, containing always DMEM/F12 (catalog\#11330032, Thermo Fisher Scientific, Waltham, MA, USA), human BDNF (10 ng/ml), human NT-3 (10 ng/ml, catalog\#450-03, PeproTech, East Windsor, NJ, USA), mouse laminin $(0.1 \mu \mathrm{g} / \mathrm{ml}$, catalog\#354232, Corning, NY, USA), N2 supplement, B27 plus supplement, non-essential amino acids (NEAA, catalog\#SH30238.01, Cytiva, Marlborough, MA, USA) supplement and doxycycline (1 mg/ml, catalog\#D9891-1G, Sigma-Aldrich, St. Louis, MO, USA) to induce TetO gene. On day 1, the culture medium was completely replaced with fresh basic media and a 48-hour puromycin selection (1 mg/l, catalog\#P8833-10MG, Sigma-Aldrich, St. Louis, MO, USA) period was started. On day 3, for calcium imaging and morphology analysis, ca. $25 \%$ mouse glia cells (prepared as previously reported $\mathrm{in}^{23}$ ) were added to the basic media plus Ara-C $(2 \mu \mathrm{M}$, catalog\#C1768-100M, Sigma-Aldrich, St. Louis, MO, USA) and 10\% mouse astrocyte conditioned media (catalog\#M1811-57, ScienCell, Carlsbad, CA, USA) to promote neuronal health and maturation. On day 5 , total medium was changed with basic media plus AraC and $10 \%$ mouse astrocyte conditioned media. On day 7 , total media was removed and replaced with BrainPhys Neuronal media with SM1 supplement (catalog\#05792, STEMCELL Technologies, Vancouver, Canada) containing always human BDNF (10 ng/ml), human NT-3 (10 $\mathrm{ng} / \mathrm{ml})$, mouse laminin $(0.1 \mu \mathrm{g} / \mathrm{ml})$, N2 supplement, doxycycline $(1 \mathrm{mg} / \mathrm{ml})$ and $10 \%$ mouse astrocyte conditioned media. From day 9 on, half of the media were removed and replaced with supplemented BrainPhys media every other day. 2.5\% FBS (catalog\#16141079, Thermo Fisher Scientific, Waltham, MA, USA) was added to the culture medium on day 11 to those cells which were co-cultured with astrocytes to support astrocyte viability. iN cells were assayed for experiments as indicated. 
Cell culture transfection: Transfection of cortical neurons at day 8 of differentiation were performed with the transfection reagent Lipofectamine 2000 (\#11668-030, Life Technologies, Carlsbad, CA, USA) as described earlier ${ }^{30}$. Cells were transfected for 48 hours with 25 pmol per well (24-well format) of miRNA mimic Pre-miR miRNA precursors (Ambion, Thermo Fisher Scientific, Waltham, MA, USA) as indicated: pre-miR Negative Control \#1 (catalog\#17110), hsamiR-185-5p (catalog\#17100, PM12486) and hsa-miR-485-5p (catalog\#17100, PM10837) or with 50 pmol per well (24-well format) of miRNA miRVana inhibitors (Ambion, Thermo Fisher Scientific, Waltham, MA, USA) as indicated: miRNA inhibitor Neg. Ctrl \#1 (catalog\#4464076), hsa-miR-1855p inhibitor (catalog\#4464084, MH12485), hsa-miR-485-5p inhibitor (catalog\#4464084, $\mathrm{MH} 10837)$.

ASOs: Emc10-targeting ASOs used in these studies were 20 bases in length, chimeric 2' -O- (2methoxyethyl) (MOE)/DNA) oligonucleotides with phosphodiester and phosphorothioate linkages. The central gap of 10 deoxynucleotides is flanked on its 5' and 3' sides by five MOE modified nucleotides. Oligonucleotides were synthesized at lonis Pharmaceuticals (Carlsbad, CA, USA) as described previously ${ }^{60,61}$. ASOs were solubilized in $0.9 \%$ sterile saline or PBS.

In vitro screening of ASOs: 4T1 cells were trypsinized, counted and diluted to 200,000 cells per $\mathrm{ml}$ in room temperature growth medium before adding $100 \mu \mathrm{L}$ of the cell suspension to the wells of a $2 \mathrm{~mm}$ electroporation plate (Harvard Apparatus, Holliston, MA, USA) which contained $11 \mu \mathrm{L}$ of 10X ASO in water. Cells were pulsed once at $130 \mathrm{~V}$ for $6 \mathrm{mS}$ with the ECM 830 instrument (Harvard Apparatus). After electroporation, the cells were transferred to a Corning Primeria 96 well culture plate (catalog \#353872, Corning, NY, USA) containing $50 \mu \mathrm{L}$ of growth medium. The cells were then incubated at $37^{\circ} \mathrm{C}$ and $5 \% \mathrm{CO} 2$. After 24 hours, the cells were washed $1 \times$ with PBS before lysing for RNA isolation and analysis. For each treatment condition duplicate wells 
were tested. ASO1081815 (TTGTTCCTACAGATCTAGGG, referred to in the manuscript as Emc10 ${ }^{\mathrm{ASO}}$ ) was used in behavioral and immunocytochemical assays.

In vivo screening of ASOs: Candidate Emc10-targeting ASOs (700ug) were stereotactically injected into the right lateral ventricle of C57BI/6 mice $(0.3 \mathrm{~mm}$ anterior, $1.0 \mathrm{~mm}$ dextrolateral, 3.0 $\mathrm{mm}$ ventral from bregma). Reduction of Emc10 mRNA in the retrosplenial cortex and thoracic spinal cord was evaluate by qRT-PCR at 2 weeks following a single bolus dose. Three ASOs were selected from the screen based on their pharmacological efficacy: 1466167, 1466171 and 1466182. Animals were injected with these ASOs in the right lateral ventricle as described above $(n=4)$ and euthanized 8 weeks post injection. Animals were evaluated with an observational functional battery test at 3 hours after dosing and then every two weeks until euthanasia. The retrosplenial cortex and thoracic spinal cord were harvested for qRT-PCR analysis of Emc10, Aif1 (microglia marker), Cd68 (phagocytic microglia marker) and Gfap (reactive astrocyte marker) mRNA. Brain and spinal cord were also harvested and fixed in formalin solution for histological evaluation. Tissues were stained for H\&E and IBA1 (microglia marker), CD68 (phagocytic microglia marker) GFAP (reactive astrocyte marker) and Calbindin (Purkinje Cell marker). Bolus injections of all three candidate ASOs resulted in similar reductions of the Emc10 mRNA at both 2 and 8 weeks. Of the three candidate ASOs, ASO1466182 (GCCATATCTTTATTAATTAC,

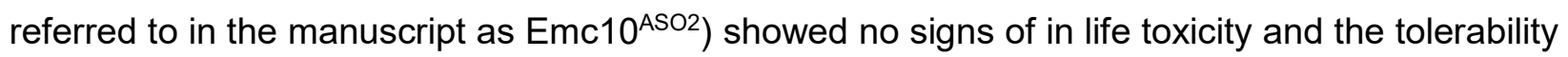
marker gene expression was similar between ASO- and vehicle-treated animals in the tissues evaluated. There was no positive IBA1 IHC staining in the CNS of any of the treated animals. Therefore, it was ranked as the best candidate for further behavioral analysis in mutant mice.

Stereotactic Intracerebroventricular (ICV) Injections of ASOs: ASOs were delivered to 8 weeks old mice via ICV injections using a Hamilton syringe (Hamilton Company, Reno, NV, USA) connected to a motorized Stoelting Quintessential Stereotaxic Injector QSI/53311 (Stoelting Co., 
Wood Dale, IL, USA). The syringe was attached to a glass pipette with a long tapered end made using a Sutter pipette puller model P-87. Anesthesia was delivered using Kent Scientific VetFlo Traditional Vaporizer VetFlo-1205S (Kent Scientific Corporation, Torrington, CT, USA). Mice were initially put in the isoflurane chamber using $3 \%$ isoflurane mixture for 5 minutes, which was lowered to 2-2.5\% when fixed to the stereotactic station. We used KOPF Small Animal Stereotaxic Instruments (Model 940). Carprofen (5 mg/kg, Zoetis Inc., Kalamazoo, MI, USA), and Bupivacaine (2 mg/kg, Hospira, Inc., Lake Forest, II, USA) were delivered subcutaneously before the incision was made. Additionally, Dexamethasone (2 mg/kg, Bimeda-MTC Animal Healt Inc., Cambridge, ON, Canada) was delivered intramuscularly. The surgical site was shaved and sterilized with betadine and $70 \%$ ethanol three times. A small midline incision was made and a hole was drilled in the skull. Stereotactic bregma coordinates used for the right ventricle were $-0.5 \mathrm{~mm}$ posterior, -1.1 lateral, and $-2.8 \mathrm{~mm}$ dorsoventral. Mice were injected with $4 \mathrm{ul}$ of either the $\mathrm{Ctrl}{ }^{\mathrm{ASO} 1 / \mathrm{ASO} 2}$ or Emc10 ${ }^{A S O 1 / A S O 2}$ (Emc10 ASO1: 292 ug, Emc10 ASO2: $280 \mathrm{ug}$ ) at a rate of 0.5ul/minute. The needle was left in the injection site for 10 minutes to allow diffusion and avoid back flow of the ASO upon retraction of the glass pipette. Mice were maintained at a temperature of $37^{\circ} \mathrm{C}$ for the duration of the surgery using a water regulated heating pad (T/Pump TP 700, Stryker Corporation, Kalamazoo, MI, USA). Mice were placed on heating pads for in cage recovery and Carprofen was administered subcutaneously for three days post-surgery. Mice were then subjected to behavioral experiments/immunohistochemistry three weeks post-surgery. qRT-PCR assays were performed one week post behavioral assays.

Quantitative Real Time PCR (qRT-PCR): Total RNA was extracted from hippocampus and PFC using the RNeasy Mini Kit (catalog\#1038703, Qiagen, Hilden, Germany) or using the miRVana miRNA isolation kit (\#AM1560, Ambion, Thermo Fisher Scientific, Waltham, MA, USA) for RNA extraction of hiPSC and derived neurons samples in accordance to their manuals. cDNA was synthesized using High-Capacity RNA-to-cDNA Kit from Applied Biosystems (cat\#4387406, 
Thermo Fisher Scientific Baltics, Vilnius, Lithuania). qRT-PCR was performed using the Bio-Rad CFX-384 qPCR instrument (Bio-Rad, Hercules, CA, USA) using TaqMan Universal Master Mix II, with UNG (catalog\#4440038, Thermo Fisher Scientific Baltics, Vilnius, Lithuania). Mouse Gapdh Endogenous Control (cat\# 4352339E, Life Technologies, Warrington, United Kingdom) served as housekeeping gene and TaqMan Mm01197551_m1 (catalog\#4351372) probe for mouse Emc10 mRNA detection were used for the qRT-PCR assay. For mouse qRT-PCR assay of Ctrl $\mathrm{ASO}^{\mathrm{ASO} 1} / \mathrm{Emc} 10^{\mathrm{ASO} 1}$, threshold cycle of each sample was picked from the linear range to calculate the values for Starting Quantity (SQ) for all samples extrapolated using the Standard Curve. All samples were run together in triplicates on the same plate including the standard curve ran in duplicates. The SQ values were averaged over the triplicates. The values of Emc10 mRNA levels were then normalized to the values from the Gapdh gene expression levels. For mouse qRT-PCR analysis of $\mathrm{Ctrl} \mathrm{ASO}^{\mathrm{ASO}} / \mathrm{Emc10} \mathrm{ASO}^{\mathrm{A}}$, the average of triplicate $\mathrm{CT}$ values from each sample was used to calculate the relative RNA levels $\left(2^{-\Delta C T}\right)$ as described earlier ${ }^{62}$ and all values were then normalized to $\mathrm{Ctrl}^{\mathrm{ASO}}$-treated WT group. qRT-PCR for hiPSC and derived neurons was performed using TaqMan or SYBR Green System (catalog\#1725121, iTaq Universal SybrGreen Supermix with ROX; BIO-RAD, Hercules, CA, USA) for mRNA and/or pre-miRNA detection according to manufacturer's instructions. U6 snRNA was used as housekeeping gene to normalize pre-miRNAs targets. For detection of DGCR14 (catalog\#4351372, Hs01574367), LZTR1 (catalog\#4331182, Hs00232479), Hs00987085), RANBP1 (catalog\# 4331182, Hs01597912), OCT4/POU5F1 (catalog\#4331182, Hs04260367), NANOG (catalog\#4331182, Hs02387400), TBR1 (catalog\#4331182, Hs00232429) and EMC10 (catalog\#4331182, Hs00382250) mRNA TaqMan probes (Thermo Fisher Scientific, Waltham, MA, USA) were used, as indicated, with GAPDH as housekeeping gene control (human GAPDH endogenous control, catalog\#4325792, Life Technologies, Warrington, United Kingdom). The average of triplicate CT values from each sample was used to calculate the relative RNA levels $\left(2^{-\Delta C T}\right)$. Primer sequences for pre-miRNA are provided in the supplemental table (Supplementary Table 3). Primers were 
purchased from Integrated DNA Technologies (Coralville, IA, USA) and were diluted to a stock concentration of $100 \mu \mathrm{M}$.

Immunohistochemistry: Animals were euthanized using $\mathrm{CO}_{2}$ and then perfused with $4 \%$ Paraformaldehyde. The brains were stored at $4^{\circ} \mathrm{C}$ in $4 \%$ PFA overnight and were then switched to 1x Phosphate Buffered Saline (PBS). 2.5\% low melting agarose in 1xPBS buffer was added to the brains placed inside the plastic molds, which were then moved to $4^{\circ} \mathrm{C}$ to create a solid block for slicing. This block was then glued to the vibratome stage (Leica Vibratome VT10005, Wetzlar, Germany) and sectioned at 40 um thickness. Sections were rinsed in 1xPBS for 5 minutes and then blocked for 1 hour at room temperature in 2\% Normal Goat Serum (NGS) and 0.3\%Triton X100 in 1 xPBS solution. Sections were then incubated with either anti-ASO and NeuN or anti-ASO and GFAP primary antibodies in blocking solution at $4^{\circ} \mathrm{C}$ and left on a shaker overnight. The following day, sections were washed three times with 1xPBS for 10 minutes and were then stained with Goat anti- rabbit along with either Goat anti-mouse or Goat anti-chicken secondary antibodies in $2 \%$ NGS and $0.4 \%$ Triton-X100 solution made in 1 XPBS for two hours in the dark at room temperature (RT). Following two 10-minute washes with 1xPBS, sections were additionally stained with Hoechst nuclear stain diluted in 1xPBS for 15 minutes in the dark. Lastly, sections were washed three times with 1xPBS for 10 minutes. Sections from PBS solution were mounted on glass slides, air-dried and cover slipped in Prolong Diamond Antifade Mountant (catalog\#P36970, Life Technologies Corporation, Eugene, OR, USA). Slides were left overnight in the dark and then stored at $4^{\circ} \mathrm{C}$. Human neuronal cultures were fixed on coverslips in $4 \%$ PFA (catalog\#22023, Biotium, Fremont, CA, USA) for $1 \mathrm{~h}$ at RT and then blocked for $1.5 \mathrm{~h}$ at RT in $0.1 \%$ Triton-X and 10\% horse serum (catalog\#H0146, Sigma-Aldrich, St. Louis, MO, USA) solution. After fixation, coverslips were stained with the primary antibody in a $0.1 \%$ Triton- $X$ and $2 \%$ horse serum solution overnight at $4^{\circ} \mathrm{C}$. Coverslips were then washed $3 \times 15$ min with DPBS (catalog\#D8537, Sigma-Aldrich, St. Louis, MO, USA) and cells were incubated for $1 \mathrm{~h}$ with the 
secondary antibody at RT followed by $3 x 15$ min DPBS washing. Tissue sections and cultured cells were imaged on W1-Yokogawa Spinning Disk Confocal (Nikon Instruments, Tokyo, Japan).

Antibodies for Immunohistochemistry: The following primary antibodies were used: Rabbit polyclonal anti-ASO antibody diluted 1:10000 (IONIS Pharmaceuticals, Carlsbad, CA, USA); AntiGFAP antibody diluted 1:1000 (Aves Labs Inc., catalog\#GFAP), Mouse monoclonal Anti-NeuN antibody diluted 1:200 (Millipore, catalog\#MAB377), anti-TUJ1 1:500 (mouse monoclonal, catalog\#T8660, Sigma-Aldrich, St. Louis, MO, USA), anti-TBR1 1:100 (rabbit monoclonal, \#Ab183022, Abcam, Cambridge, MA, USA), anti-GFP 1:1000 (goat polyclonal, catalog\#600-101215, Rockland Immunochemicals, Pottstown, PA, USA), anti-MAP2 1:2000 (chicken polyclonal, catalog\#5392, Abcam, Cambridge, MA, USA). The following secondary antibodies were used for mouse at a dilution of 1:500: Goat anti-Rabbit (Alexa Floro 488: catalog\#AA1008, Invitrogen, Waltham, MA, USA) against ASO; Goat anti-mouse (Alexa Floro 568: catalog\#AA1004, Invitrogen, Waltham, MA, USA) against NeuN, and Goat anti chicken (Alexa Floro 568: catalog\#A11041, Invitrogen, Waltham, MA, USA) against GFAP. Hoechst 33258 solution diluted 1:1000 (Catalog\#94403, Sigma Aldrich, Saint Louis, MO, USA) was used for nuclear staining in brain slices and DAPI Fluoromount-G (catalog\#0100-20, Southern Biotech, Birmingham, AL, USA) was used for cultured cells.

Analysis of dendritic complexity: iNs were prepared on coverslips, fixed at DIV21 and immunostained for TBR1 and MAP2 (as described above) to identify dendritic branches. Images of dendrites were acquired on a Nikon Spinning Disk Confocal Microscope and captured using the Nikon NIS Elements AR (v.5.21.03 64-bit) software. Images were acquired and analyzed as previously described ${ }^{7,63}$. Dendrite branches were semi-automatically traced using NeuronStudio software (v.0.9.92 64-bit) ${ }^{64}$. The output .swc files were then processed in VAA3D (v.3.1.00) ${ }^{65-67}$ 
and binary images were generated and analyzed using ImageJ (http://rsbweb.nih.gov/ij/, NIH, Bethesda, MD, USA).

Calcium imaging: Calcium imaging was performed as previously described ${ }^{68}$ with a few modifications. Neuronal cells used in calcium imaging experiments were prepared on glass bottom dishes (14mm, catalog\#P35G-1.5-14-C, MatTek, Ashland, MA, USA). Briefly, cells were

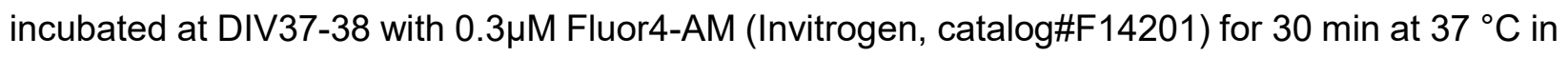
incubation buffer medium (containing $170 \mathrm{mM} \mathrm{NaCl}^{3} 3.5 \mathrm{mM} \mathrm{KCl}, 0.4 \mathrm{mM} \mathrm{KH}_{2} \mathrm{PO}_{4}, 20 \mathrm{mM}$ TES (Ntris[hydroxyl-methyl-2-aminoethane-sulfonic acid], $5 \mathrm{mM} \mathrm{NaHCO}_{3}, 5 \mathrm{mM}$ glucose, 1.2 $\mathrm{mM} \mathrm{Na}_{2} \mathrm{SO}_{4}$, 1.2mM $\left.\mathrm{MgCl}_{2}, 1.3 \mathrm{mM} \mathrm{CaCl} 2, \mathrm{pH} 7.4\right)$ and washed once with incubation buffer medium before imaging. After imaging for 2'20" (baseline), $25 \mathrm{mM} \mathrm{KCl}$ solution was added to the cells, followed by another $25 \mathrm{mM} \mathrm{KCl}$ addition at 3'20" and 4'20" min. Live imaging was performed at room temperature $\left(\sim 25^{\circ} \mathrm{C}\right)$ on a W1-Yokogawa Spinning Disk Confocal (Nikon Instruments, Tokyo, Japan). ImageJ software with plugin for motion correction (MuliStackReg) and Excel were used to collect, manage and quantify time-lapse excitation ratio images by selecting cell body as ROI.

Protein extraction for Western blot: To extract proteins from mouse hippocampus, the tissue was dissected and homogenized in QIAzol lysis reagent (catalog\#79306, Qiagen, Hilden, Germany). Chloroform was added to homogenate and the solution was then incubated at room temperature for 3 minutes. Tissue was spun at $12,000 \times \mathrm{g}$ at $4^{\circ} \mathrm{C}$ and the organic phase was collected for protein extraction. We followed an optimized protocol for protein extraction from Trizol solutions and used 5\% SDS + 20mM EDTA + $140 \mathrm{mM} \mathrm{NaCl}$ buffer solution for protein pellet suspension ${ }^{69}$. Cultured neurons cells were lysed at day 8 of differentiation. The cultured cells were once washed with cold DPBS (catalog\#D8537, Sigma-Aldrich, St. Louis, MO, USA). Cells were then lysed by adding a modified Pierce RIPA lysis and extraction buffer (RIPA+) (catalog\#89900, Thermo Scientific, Rockford, IL, USA) that contains a Halt Protease Inhibitor Cocktail 
(catalog\#1861281, Thermo Scientific, Rockford, IL, USA). The plate was shaking for $20 \mathrm{~min}$ at $4^{\circ} \mathrm{C}$ on an orbital shaker (catalog\#980173, Talboys, Troemner, Thorofare, NJ, USA). To remove cell debris, the lysates were centrifuged at maximum speed for $10 \mathrm{~min}$ at $4^{\circ} \mathrm{C}$. The protein concentration of the supernatant for all protein samples was determined by Pierce BCA Protein Assay Kit (\#23227, Thermo Scientific, Rockford, IL, USA).

Western Blots: For each lane, $\sim 20 \mu \mathrm{g}$ protein were run on a 4-12Bis-Tris Criterion XT Precast Gel (\#3450123, Bio-Rad (Bio-Rad, Hercules, CA, USA) next to the Precision Plus Protein Dual Color Standard (catalog\#161-0374, Bio-Rad, Hercules, CA, USA) in SDS-PAGE running buffer (catalog\#1610788, Bio-Rad, Hercules, CA, USA) and afterwards transferred to a methanolactivated Immobilon-P PVDF (poly-vinylidene difluoride) membrane (catalog\#IPCVH00010, Merck Millipore Ltd., Carrigtwohill, Ireland) by tank blotting at 250mA for $90 \mathrm{~min}$ in a cold room (4 ${ }^{\circ} \mathrm{C}$ ) in blotting buffer (catalog\#1610734, Bio-Rad, Hercules, CA, USA). The membrane was blocked for $2 \mathrm{~h}$ in TBS-T (tris buffered saline supplemented with $0.1 \%$ Tween) containing $5 \%$ milk powder. Antibody dilutions anti-EMC10/Emc10 1:1000 (rabbit polyclonal; catalog\#Ab181209, Abcam, Cambridge, MA, USA ), anti-DGCR8 1:1000 (rabbit monoclonal, catalog\#Ab191875, Abcam, Cambridge, MA, USA), anti-RANBP1 1:500 (rabbit polyclonal, catalog\#Ab97659, Abcam, Cambridge, MA, USA) and anti-alpha-Tubulin1:1000 (polyclonal rabbit; catalog\#2144S, Cell Signal, Danvers, MA, USA) as loading control were prepared in TBS-T/milk and the membrane were incubated overnight at $4^{\circ} \mathrm{C}$ under slight shaking on an orbital shaker (catalog\#980173, Talboys, Troemner, Thorofare, NJ, USA). After three washes with TBS-T, the membrane was incubated with LI-COR goat anti-Rabbit antibody IRDye 800CW (catalog\#925-32211, LI-COR Bioscience, Lincoln, NE, USA) for 1.5h at RT. After three washes with TBS-T the membrane was developed using the LI-COR Odyssey CLx system (LI-COR Bioscience, Lincoln, NE, USA) using LI-COR Image Studio software (Ver.5.2) and quantification of band intensity was performed by ImageJ (NIH, Bethesda, MD, USA). 
Bulk RNAseq and bioinformatic analysis of mouse hippocampal samples: Total RNA was isolated from 4 WT Ctrl ${ }^{\mathrm{ASO} 1}, 4$ WT Emc10 ${ }^{\mathrm{ASO}}, 5 \mathrm{Df} 16 \mathrm{Ctrl}^{\mathrm{ASO} 1}$ and $4 \mathrm{Df} 16 \mathrm{Emc10}{ }^{\mathrm{ASO} 1}$ treated male hippocampi. Stranded polyA+ enriched RNA sequencing libraries were prepared at the Columbia Genome Center (Columbia University, New York, USA) and raw passing filters reads were sequenced on Illumina Novaseq 6000 instrument using STRYPOLYA library prep kit (uses a polyA pull down method) with 40 million reads. Sequence reads were aligned to the mouse genome (Ensembl, GRCm38) using the STAR sequence alignment tool (version 2.7) ${ }^{70}$ and gene count matrices were generated. Differential gene expression was analyzed using the DESeq2 pipeline ${ }^{71}$ in $\mathrm{R}$ and volcano plots were generated using the open source Enhanced Volcano package in $\mathrm{R}$ (https://github.com/kevinblighe/EnhancedVolcano).

Bulk RNAseq and small RNA/miRNAseq of hiPSC-derived cortical neurons: Total RNA was extracted from hiPSC-derived cortical neurons at DIV8 (Q5, Q6, n=3 each group) using the miRVana miRNA isolation kit (\#AM1560, Ambion, Thermo Fisher Scientific, Waltham, MA, USA) in accordance to their manuals. For bulk RNAseq, Poly(A) RNA sequencing library was prepared following Illumina's TruSeq-stranded-mRNA (Illumina, San Diego, CA, USA) sample preparation protocol. RNA integrity was checked with Bioanalyzer 2100 (Agilent, CA, USA). Poly(A) tailcontaining mRNAs were purified using oligo-(dT) magnetic beads with two rounds of purification. After purification, poly(A) RNA was fragmented using divalent cation buffer in elevated temperature. Quality control analysis and quantification of the sequencing library were performed using Agilent Technologies 2100 Bioanalyzer High Sensitivity DNA Chip (Agilent, CA, USA). Paired-ended sequencing was performed on Illumina's NovaSeq 6000 (LC Sciences, Houston, TX, USA) sequencing system. For miRNAseq, total RNA quality and quantity was analyzed with Bioanalyzer 2100 (Agilent, CA, USA), with RIN number >7.0. Approximately 1 ug of total RNA was then used to prepare small RNA library according to the protocol of TruSeq Small RNA 
Sample Prep Kits (Illumina, San Diego, CA, USA). Then, a single-end sequencing 50bp on an Illumina Hiseq 2500 following the vendor's recommended protocol was conducted.

Bioinformatics analysis of human neuron bulk RNAseq: Cutadapt ${ }^{72}$ and in house perl scripts were used to remove the reads that contained adaptor contamination, low quality bases and undetermined bases. Sequence quality was subsequently verified using FastQC (http://www.bioinformatics.babraham.ac.uk/projects/fastqc/). HISAT2 ${ }^{73}$ was used to map reads to the genome of ftp://ftp.ensembl.org/pub/release-101/fasta/homo_sapiens/dna/. The mapped reads of each sample were assembled using String $\mathrm{Tie}^{74}$. Then, all transcriptomes were merged to reconstruct a comprehensive transcriptome using perl scripts and GffCompare. After the final transcriptome was generated, StringTie and edge $\mathrm{R}^{75}$ were used to estimate the expression levels of all transcripts. StringTie was used to assess expression levels for mRNAs by calculating FPKM. The differentially expressed mRNAs were selected with log2 (fold change) >1 or log2 (fold change) $<-1$ and with statistical significance ( $p$ value $<0.05$ ) by $R$ package edgeR. For the VolcanoPlot visualization, the web-based $\mathrm{R}$ package Shiny application "VolcanoPlot" (https://paolo.shinyapps.io/ShinyVolcanoPlot/) was used. DEGs (adj. Pvalue<0.05) were plotted by selecting the axes ((Log2(FC) range $=-2.5 / 2.5$ and - Log10(Pvalue $)=15)$ and setting the cutoff selection (P-value threshold=1.3 (0.05), Log2(FC) threshold 0.4 and 3). The GO-Term enrichment analysis was performed for the up- and downregulated protein-coding genes (1937/2094, DEG) by using the standard setting (g:SCS threshold) of the gProfiler webtool (https://biit.cs.ut.ee/gprofiler/gost) ${ }^{76}$. TargetScan (v8.0, http://www.targetscan.org/vert_80/) was used for miRNA binding site prediction ${ }^{77}$. Intersection of genes and predicted targets were conducted by using the VIB / UGent Bioinformatics \& Evolutionary Genomics (Gent, Belgium) webtool "Venn" (https://bioinformatics.psb.ugent.be/webtools/Venn/). 
Bioinformatics analysis of human neuron miRNA-seq: Raw reads were subjected to an inhouse program, ACGT101-miR (LC Sciences, Houston, TX, USA) to remove adapter dimers, junk, low complexity, common RNA families (rRNA, tRNA, snRNA, snoRNA) and repeats. Subsequently, unique sequences with length in 18 26 nucleotide were mapped to specific species precursors in miRBase (v22.0) by BLAST search to identify known miRNAs and novel 3p- and 5p- derived miRNAs. Length variation at both 3' and 5' ends and one mismatch inside of the sequence were allowed in the alignment. The unique sequences mapping to specific species mature miRNAs in hairpin arms were identified as known miRNAs. The unique sequences mapping to the other arm of known specific species precursor hairpin opposite to the annotated mature miRNA-containing arm were considered to be novel $5 p$ - or $3 p$ derived miRNA candidates. The remaining sequences were mapped to other selected species precursors (with the exclusion of specific species) in miRBase 22.0 by BLAST search, and the mapped pre-miRNAs were further BLASTed against the specific species genomes to determine their genomic locations. The above two mentioned mapped $5 p$ and $3 p$ sequences were defined as known miRNAs. The unmapped sequences were BLASTed against the specific genomes, and the hairpin RNA structures containing sequences were predicted from the flank 80 nt sequences using RNAfold (http://rna.tbi.univie.ac.at/cgi-bin/RNAWebSuite/RNAfold.cgi) software. The criteria for secondary structure prediction were: (1) number of nucleotides in one bulge in stem $(\leq 12)(2)$ number of base pairs in the stem region of the predicted hairpin $(\geq 16)(3)$ cutoff of free energy $(\mathrm{kCal} / \mathrm{mol} \leq-$ $15)$ (4) length of hairpin (up and down stems + terminal loop $\geq 50$ ) (5) length of hairpin loop ( $\leq 20)$. (6) number of nucleotides in one bulge in mature region $(\leq 8)(7)$ number of biased errors in one bulge in mature region $(\leq 4)(8)$ number of biased bulges in mature region $(\leq 2)(9)$ number of errors in mature region $(\leq 7)(10)$ number of base pairs in the mature region of the predicted hairpin $(\geq 12)$ (11) percent of mature in stem $(\geq 80)$. For the VolcanoPlot visualization, the web-based $R$ package Shiny application "VolcanoPlot" (https://paolo.shinyapps.io/ShinyVolcanoPlot/) was used. DEmiRs ( Pvalue $<0.05)$ of known miRNAs were plotted by selecting the axes $(($ Log2 $(F C)$ range $=$ 
$-5 / 5$ and $-\log 10($ Pvalue $=10)$ and setting the cutoff selection (P-value threshold=1.3 (0.05), Log2(FC) threshold 0.4). For the GO-term enrichment analysis of the up- and down-regulated miRNAs (153/133, DEmiRs) the webtool miRNet 2.0 (https://www.mirnet.ca/) with standard settings (tissue: nervous, targets: genes [miRTarBase 8.0]) was used ${ }^{34}$. TargetScan (v8.0, http://www.targetscan.org/vert_80/) was used for all miRNA binding site prediction ${ }^{77}$ for miR-185$5 p$, miR1306-5p and miR-1286 and using the biochemical predicted occupancy model ${ }^{78}$ for table sorting.

Behavioral assays: The experimenter was blind to mouse genotype and treatments while performing behavioral assays and data analysis. Mice were 11-15 weeks old at the time of allbehavioral testing and three weeks post the surgical delivery of ASOs.

Social Memory assay: Assays were performed as described earlier in more details ${ }^{19}$. All experimental mice were single housed, moved to the testing room one hour prior to testing and returned to their home cages after the completion of the experiment. Both male and female were tested for ASO1 and only males were tested for ASO2. Stimulus mice (C57 BL/6J) were obtained from Jackson Labs. All stimulus mice were between the ages of 3-4 weeks. Test and stimulus mice were sex matched in the experimental trials. All trials were recorded using a video camera (Webcam Pro 9000, Logitech, Lausanne, Switzerland) and recorder software (Logitech video recording software). Stimulus mice were color marked on the tails to distinguish the stimulus mice from the test mice, during video analysis. The videos were manually scored for total interaction time over the course of the trials (5 minutes) for interactions initiated by the test animal including anal sniffing, nose-to-nose touch and close following. For the novel/familiar paradigm, test and stimulus (novel) mice were placed together in a neutral cage and the interaction was recorded for 5 minutes (trial 1). One hour after trial 1, the same stimulus (familiar) mouse was placed together with the test mouse and the interaction was recorded for another 5 minutes (trial 2). A similar procedure was followed for the control novel/novel paradigm, except that we used different 
stimulus mice for trial 1 and trial 2 such that at trial 2 the stimulus mice were also novel for the experimental mice. Trials with experimental mice showing highly aggressive behavior towards the stimulus mice were stopped and the animals were excluded from the analysis.

Contextual Fear Conditioning assay: Contextual Fear Conditioning assays were performed as described earlier ${ }^{5,19}$ using an Coulbourn animal shocker (Model H13-15 110V, Coulbourn Instruments, Whitehall, PA, USA). Sound levels were checked with a Digital Sound Level Meter (Model: 407730, Extech Instruments, MA, USA) before beginning the trials. Using a cotton swab, pure lemon extract (McCormick \& Co, Hunt Valley, MD, USA) was introduced into the testing chamber adding 9 different but equal distributed spots on a napkin. Test mice were placed in the test chamber and received 2 pairs of a tone $(30 \mathrm{~s}, 82 \mathrm{db})$ and a co-terminating shock $(2 \mathrm{~s}, 0.7 \mathrm{~mA})$. Mice were then carefully picked with forceps and returned to their home cage. After 24 hours, mice were placed in the FC box again with same environment and lemon scent for 6 minutes in the absence of tone and shock to test for contextual memory. The box and grid were cleaned with $70 \% \mathrm{EtOH}$ before and between every test run on both days. Videos were recorded and analyzed by using FreezeFrame 3 software (Harvard Apparatus, Holliston, MA, USA).

Statistical Analysis: Data were analyzed using GraphPad Prism (Graphpad Software, Inc., San Diego, CA, USA). Data were evaluated as indicated, using either unpaired two-tailed t-test, Kolmogorov-Smirnov test, one-way, two-way or three-way analysis of variance (ANOVA) tests followed by post hoc Tukey's multiple comparison test for comparisons across all groups. Data are presented as mean \pm SEM. $P$ values for each comparison are described in the figure legends, $P<0.05$ was considered as statistically significant $\left({ }^{*} P<0.05 ;{ }^{* *} P<0.01 ;{ }^{* * *} P<0.001\right.$; $* * * * P<0.0001)$ 


\section{References}

52 Li, Y. et al. Investigation of Neurodevelopmental Deficits of 22 q11.2 Deletion Syndrome with a Patient-iPSC-Derived Blood-Brain Barrier Model. Cells 10, doi:10.3390/cells10102576 (2021).

53 Lin, M. et al. Integrative transcriptome network analysis of iPSC-derived neurons from schizophrenia and schizoaffective disorder patients with 22q11.2 deletion. BMC Syst Biol 10, 105, doi:10.1186/s12918-016-0366-0 (2016).

54 Fusaki, N., Ban, H., Nishiyama, A., Saeki, K. \& Hasegawa, M. Efficient induction of transgene-free human pluripotent stem cells using a vector based on Sendai virus, an RNA virus that does not integrate into the host genome. Proc Jpn Acad Ser B Phys Biol Sci 85, 348-362, doi:10.2183/pjab.85.348 (2009).

55 Riera, M. et al. Generation of two iPS cell lines (FRIMOi003-A and FRIMOi004-A) derived from Stargardt patients carrying ABCA4 compound heterozygous mutations. Stem Cell Res 36, 101389, doi:10.1016/j.scr.2019.101389 (2019).

56 Cradick, T. J., Qiu, P., Lee, C. M., Fine, E. J. \& Bao, G. COSMID: A Web-based Tool for Identifying and Validating CRISPR/Cas Off-target Sites. Mol Ther Nucleic Acids 3, e214, doi:10.1038/mtna.2014.64 (2014).

57 Lee, G. et al. Modelling pathogenesis and treatment of familial dysautonomia using patient-specific iPSCs. Nature 461, 402-406, doi:10.1038/nature08320 (2009).

58 Hockemeyer, D. et al. A drug-inducible system for direct reprogramming of human somatic cells to pluripotency. Cell Stem Cell 3, 346-353, doi:10.1016/j.stem.2008.08.014 (2008).

59 Ho, S. M. et al. Evaluating Synthetic Activation and Repression of NeuropsychiatricRelated Genes in hiPSC-Derived NPCs, Neurons, and Astrocytes. Stem Cell Reports 9 , 615-628, doi:10.1016/j.stemcr.2017.06.012 (2017).

60 Cheruvallath, Z. S., Cole, D. L. \& Ravikumar, V. T. A novel solid support for synthesis of oligonucleotide 3'-phosphorothioate monoesters. Bioorg Med Chem Lett 13, 281-284, doi:10.1016/s0960-894x(02)00922-8 (2003).

61 McKay, R. A. et al. Characterization of a potent and specific class of antisense oligonucleotide inhibitor of human protein kinase C-alpha expression. J Biol Chem 274, 1715-1722, doi:10.1074/jbc.274.3.1715 (1999).

62 Brenes, J. C. et al. Differential effects of social and physical environmental enrichment on brain plasticity, cognition, and ultrasonic communication in rats. J Comp Neurol 524, 1586-1607, doi:10.1002/cne.23842 (2016).

63 Mukai, J. et al. Palmitoylation-dependent neurodevelopmental deficits in a mouse model of 22q11 microdeletion. Nat Neurosci 11, 1302-1310, doi:10.1038/nn.2204 (2008).

64 Wearne, S. L. et al. New techniques for imaging, digitization and analysis of threedimensional neural morphology on multiple scales. Neuroscience 136, 661-680, doi:10.1016/j.neuroscience.2005.05.053 (2005).

65 Peng, H., Ruan, Z., Long, F., Simpson, J. H. \& Myers, E. W. V3D enables real-time 3D visualization and quantitative analysis of large-scale biological image data sets. Nat Biotechnol 28, 348-353, doi:10.1038/nbt.1612 (2010).

66 Peng, H., Bria, A., Zhou, Z., Iannello, G. \& Long, F. Extensible visualization and analysis for multidimensional images using Vaa3D. Nat Protoc 9, 193-208, doi:10.1038/nprot.2014.011 (2014). 
67 Peng, H. et al. Virtual finger boosts three-dimensional imaging and microsurgery as well as terabyte volume image visualization and analysis. Nat Commun 5, 4342, doi:10.1038/ncomms5342 (2014).

68 Barreto-Chang, O. L. \& Dolmetsch, R. E. Calcium imaging of cortical neurons using Fura-2 AM. J Vis Exp, doi:10.3791/1067 (2009).

69 Kopec, A. M., Rivera, P. D., Lacagnina, M. J., Hanamsagar, R. \& Bilbo, S. D. Optimized solubilization of TRIzol-precipitated protein permits Western blotting analysis to maximize data available from brain tissue. J Neurosci Methods 280, 64-76, doi:10.1016/j.jneumeth.2017.02.002 (2017).

70 Dobin, A. et al. STAR: ultrafast universal RNA-seq aligner. Bioinformatics 29, 15-21, doi:10.1093/bioinformatics/bts635 (2013).

71 Love, M. I., Huber, W. \& Anders, S. Moderated estimation of fold change and dispersion for RNA-seq data with DESeq2. Genome Biol 15, 550, doi:10.1186/s13059-014-0550-8 (2014).

72 Martin, M. Cutadapt removes adapter sequences from high-throughput sequencing reads. 2011 17, 3, doi:10.14806/ej.17.1.200 (2011).

73 Kim, D., Langmead, B. \& Salzberg, S. L. HISAT: a fast spliced aligner with low memory requirements. Nat Methods 12, 357-360, doi:10.1038/nmeth.3317 (2015).

74 Pertea, M. et al. StringTie enables improved reconstruction of a transcriptome from RNA-seq reads. Nat Biotechnol 33, 290-295, doi:10.1038/nbt.3122 (2015).

75 Robinson, M. D., McCarthy, D. J. \& Smyth, G. K. edgeR: a Bioconductor package for differential expression analysis of digital gene expression data. Bioinformatics 26, 139140, doi:10.1093/bioinformatics/btp616 (2010).

76 Raudvere, U. et al. g:Profiler: a web server for functional enrichment analysis and conversions of gene lists (2019 update). Nucleic Acids Res 47, W191-W198, doi:10.1093/nar/gkz369 (2019).

77 Agarwal, V., Bell, G. W., Nam, J. W. \& Bartel, D. P. Predicting effective microRNA target sites in mammalian mRNAs. Elife 4, doi:10.7554/eLife.05005 (2015).

78 McGeary, S. E. et al. The biochemical basis of microRNA targeting efficacy. Science 366, doi:10.1126/science.aav1741 (2019). 


\section{Supplementary Information}

Figure S1: Validation and characterization of hiPSCs and hiPSC-derived neurons. (a) Characterization of Q5 (Ctrl) and Q6 (22q11.2) hiPS cells demonstrate normal karyotype distribution in both lines. (b-d) Characterization of the Q5 and Q6 hiPSC lines. Multiplex Ligationdependent Probe Amplification (MLPA) assay of gene copies within and around 22q11.2 locus for Q5 (Ctrl) (b) and Q6 (22q11.2) (c) line shows that copy number of genes in the 22q11.2 locus (highlighted in light blue) are reduced by half in the Q6 (22q11.2) hiPSC line. (d) qRT-PCR assays of embryonic stem cell marker OCT4/POU5F1 and NANOG shows that they are highly expressed in both hiPSC lines (Q5 Ctrl and Q6 22q11.2). (e) TBR1 and TUJ1 expression of cortical neurons by immunocytochemistry at day 20 of differentiation indicates the efficiency of hiPSC differentiation into cortical neurons. TBR1 (red), TUJ1 (green) and DAPI (blue). Scale bar: 100 $\mu \mathrm{m}$. (f-g) Time-course qRT-PCR analysis at day 0 and day 20 of differentiation of human pluripotency marker (f) OCT4/POU5F1 $(\mathrm{P}=0.0012)$ and $(\mathbf{g})$ TBR1 $(\mathrm{P}=0.0273)$, a preplate, subplate and cortical Layer VI neuron marker in Q5 (Ctrl) line. Day 0: n=4, Day 20 n=4. Data are shown as mean \pm SEM, unpaired two-tailed t-test, ${ }^{*} p<0.05,{ }^{* *} p<0.01$.

Figure S2: Expression profile of 22q11.2 locus genes and EMC10 in cortical neurons at day 8 of differentiation. FPKM values are shown for (a) 22q11.2 locus genes and (b) $19 q 13$ locus gene EMC10 (Q5 n=3, Q6 n=3). Data are presented as mean \pm SEM, adj. $P<0.05$.

Figure S3: Expression profile of selected miRNAs in Q5 (Ctrl) and Q6 (22q11.2) cortical neurons at day 8 of differentiation. (a) 22q11.2 region residing miRNAs and (b) selected miRNAs residing outside the $22 q 11.2$ region (Q5 $n=3, Q 6 n=3)$. Data are presented as mean \pm SEM, $P<0.05$ 
Figure S4: Predicted miRNA targets. (a) Schematic of human and mouse Emc10 3'UTR showing miR-185 (green) and miR-485 (blue) binding sites predicted by TargetScan. Conserved target sites in mammals are marked with an asterisk. Specific binding site positions in Emc10

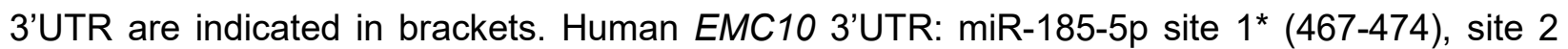
(2807-2813), miR-485-5p site $1^{*}(473-479)$, site 2 (585-591), site 3 (1530-1536), site 4 (19962002), site 5 (3516-3522), site 6 (4158-4164). Mouse Emc10 3'UTR: miR-185-5p site 1* (400407), site 2 (454-460), miR-485-5p site $1^{*}(406-412)$, site 2 (513-519), site 3 (796-802), site 4 (2842-2848), site 5 (4013-4020), site 6 (4576-4582). (b) Venn diagram highlighting shared predicted targets of 22q11.2 locus residing miRNAs miR-185-5p, miR-1286 and miR-1306-5p. (c) Venn diagram highlighting shared predicted targets of all three miR-185-5p, miR-1286 and miR1306-5p among upregulated DEGs. Identified are top predicted targets that carry at least one conserved miRNA binding site.

Figure S5: Characterization of hiPSC lines carrying EMC10 LoF mutations. (a) CRISPR/Cas9 genome editing in Q6 (22q11.2) hiPSCs to obtain EMC10 LoF mutation lines. Two 20bp guided RNAs (gRNAs) were designed to specifically target the EMC10 exon 3 region (transcript 201, ENNST00000334976.11), to generate a frameshift mutation as indicated by the green (EMC10-g1) and blue (EMC10-g2) arrow in the gRNA profiles at EMC10 locus (Fig. S5a, upper panel). Upper panel (Fig. S5a): gRNA design and profile at the targeted EMC10 locus that leads to frameshift mutations. Lower panel (Fig. S5a): Genotyping of positive clones for Q6/EMC10 $0^{\mathrm{HET}}$ and Q6/EMC10 ${ }^{\mathrm{HOM}}$ lines confirms frameshift mutation. (b) Characterization of Q6/EMC10 $10^{\mathrm{HET}}$ and Q6/EMC10 ${ }^{\mathrm{HOM}}$ hiPS cells demonstrate normal karyotype distribution. (c-f) qRT-PCR assay in hiPSC lines. Embryonic stem cell marker (c) NANOG and (e) OCT4/POU5F1 are highly expressed in all four hiPSC lines assayed. (e-f) Reduced mRNA expression of 22q11.2 region genes in 22q11.2DS/SCZ related hiPSC lines. (e) DGCR14 is reduced in Q6 (P=0.0005), Q6/EMC10 ${ }^{\mathrm{HET}}(\mathrm{P}<0.0001)$ and $\mathrm{Q6/EMC10}{ }^{\mathrm{HOM}}$ lines $(\mathrm{P}<0.0003)$ compared to $\mathrm{Q} 5(\mathrm{Ctrl})$ line. (f) 
LZTR1. is reduced in Q6 (P=0.0089), Q6/EMC10 ${ }^{\mathrm{HET}}(\mathrm{P}<0.0001)$ and $\mathrm{Q6/EMC10^{ \textrm {HOM } }}$ lines $(P=0.0285)$ compared to $Q 5(C \operatorname{trl})$ line. $Q 5(C \operatorname{trl}) n=4-5, Q 6(22 q 11.2) n=4=5, Q 6 / E M C 10^{H E T} n=6$, Q6/EMC10 ${ }^{\mathrm{HOM}} \mathrm{n}=5$. (g) Western blot analysis showing reduction (Q6/EMC10 ${ }^{\mathrm{HET}}$ ) or elimination $\left(\mathrm{Q} 6 / \mathrm{EMC} 10^{\mathrm{HOM}}\right)$ of EMC10 protein levels in the EMC10 LoF mutant hiPSC lines. Tubulin was probed as a loading control. Data are shown as mean \pm SEM, unpaired two-tailed t-test, ${ }^{*} \mathrm{P}<0.05$, ${ }^{* *} \mathrm{P}<0.01,{ }^{* * *} \mathrm{P}<0.001,{ }^{* * *} \mathrm{P}<0.0001$

Figure S6: In vivo screening of in silico designed ASOs. (a-c) In vivo efficacy of selected ASOs in suppressing the levels of Emc10 mRNA in the cortex of WT mice at 2- and 8-weeks post injection. Three ASOs were selected from an initial screen for the analysis shown here, based on their pharmacological efficacy. 700 ug of ASOs were injected into the right lateral ventricle of C57BI/6 mice. Depicted is their efficacy in suppressing the levels of Emc10 mRNA in the (a) retrosplenial cortex (cortex) and (b) thoracic spinal cord (TSC) at both 2- and 8-wks following a single bolus dose. (c) Calculation of the approximate time to normal expression ( T100\%) from the 2- and 8-week Emc10 expression data. T100\% for Emc10 \#1466182 ASO is 26 weeks but this remains to be empirically determined.

Supplementary Table S1: Clinical and demographic characteristics of 22q11.2DS/SCZ patients and healthy controls.

Supplementary Table S2: Predicted targets of miR-185-5p, miR-1286 and miR-1306-5p in EMC10 3'UTR.

Supplementary Table S3: List of primer sequences used for qRT-PCRs.

Supplementary Table S4: List of genes upregulated in $\left.\mathrm{Ctr}\right|^{\mathrm{ASO} 1}$ treated-Df(16) $A^{+/-}$mice compared to $\mathrm{Ctr}{ }^{\mathrm{ASO} 1}$ treated-WT mice but not in the Emc10 ${ }^{\mathrm{ASO} 1}$ treated-Df(16) $A^{+/-}$compared to Emc10-ASO1 treated-WT mice. 
bioRxiv preprint doi: https://doi.org/10.1101/2022.03.01.482495; this version posted March 4, 2022. The copyright holder for this preprint (which was not certified by peer review) is the author/funder. All rights reserved. No reuse allowed without permission.

a

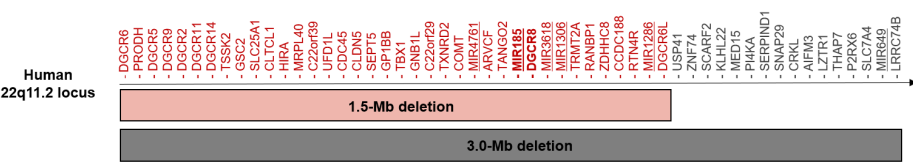

b

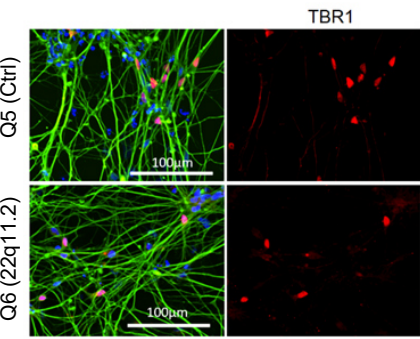

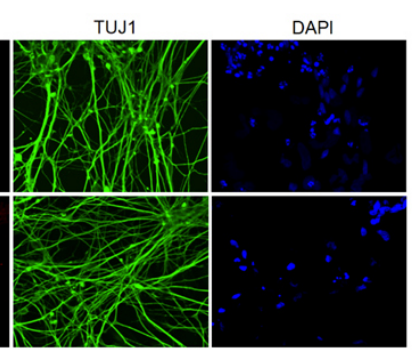

C
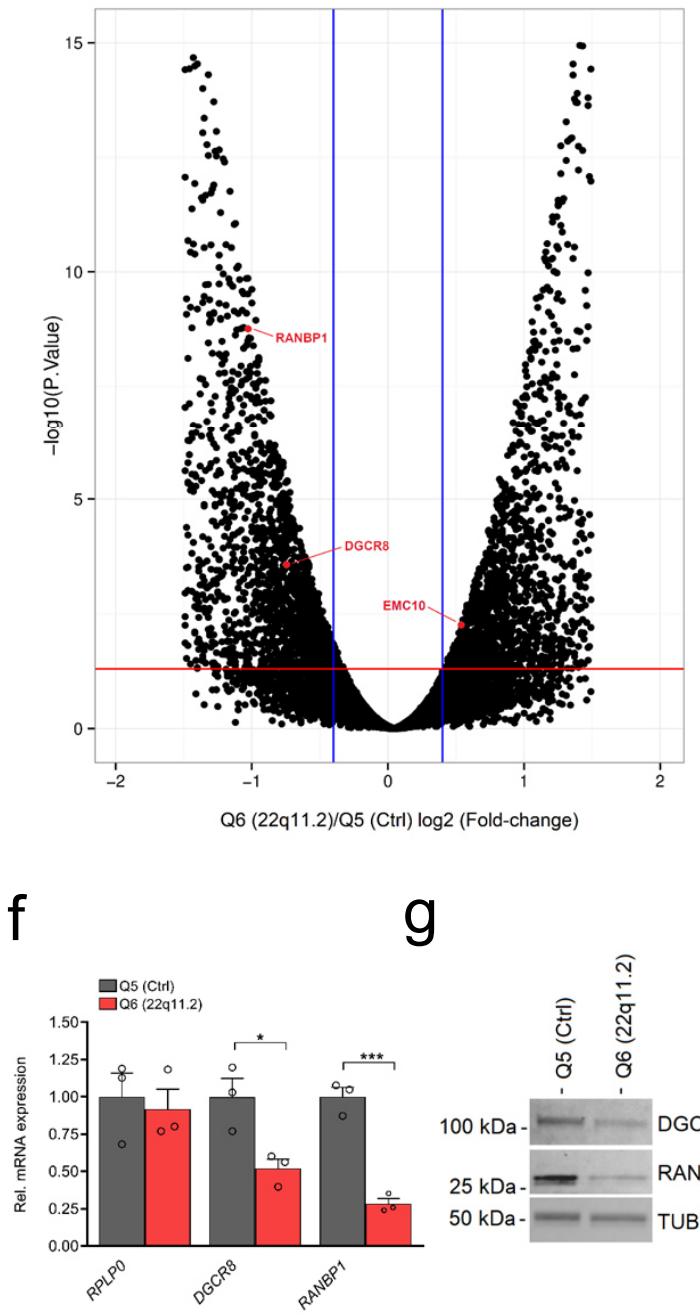

g

k

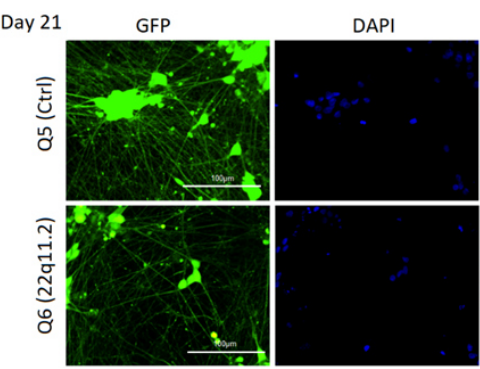

TBR1 d

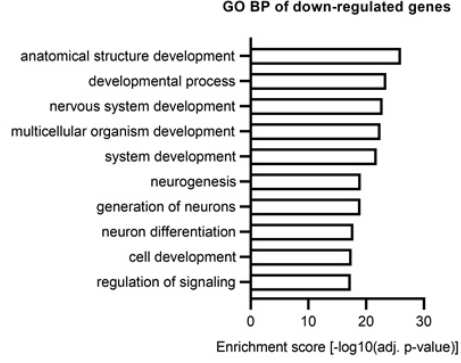

e
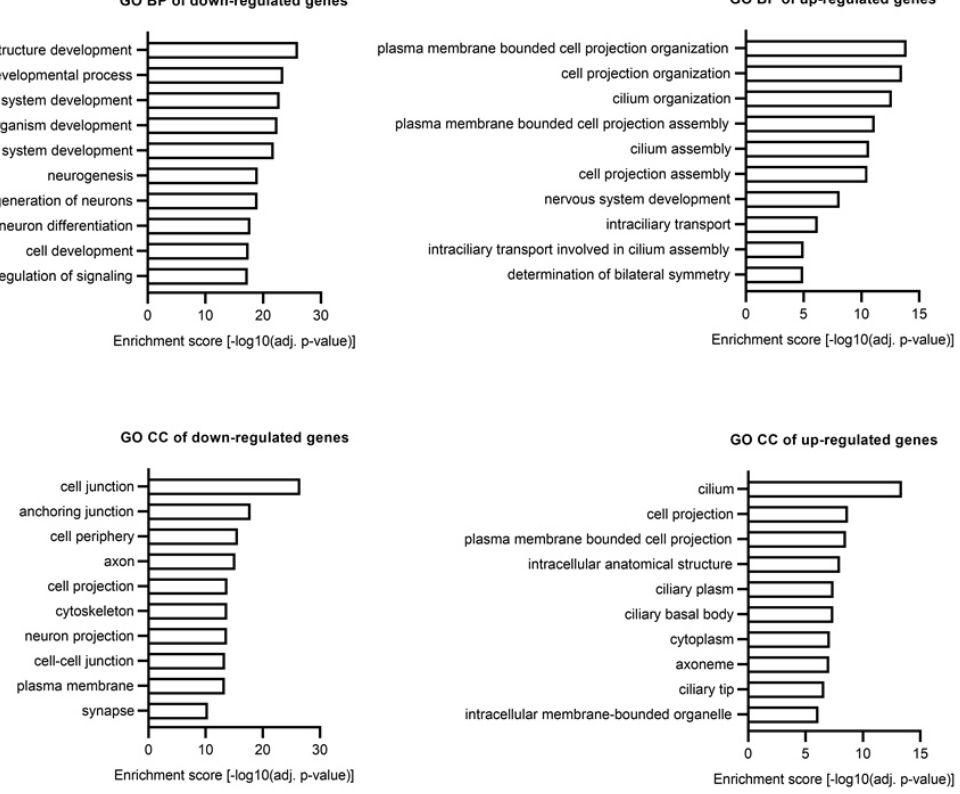

h

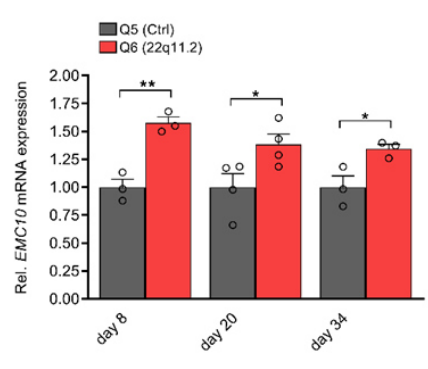

i
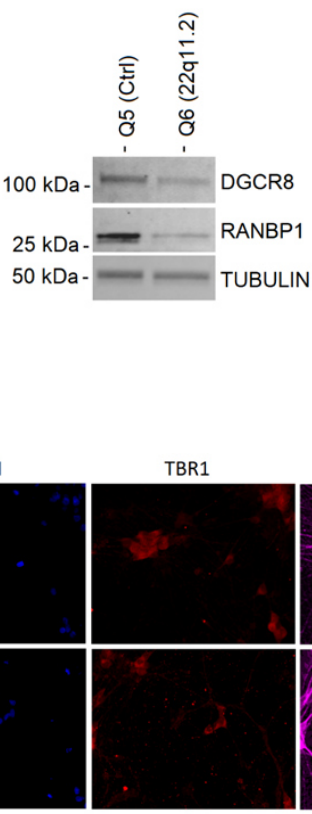

MAP2

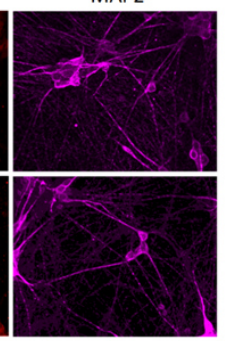

I

$\mathrm{m}$

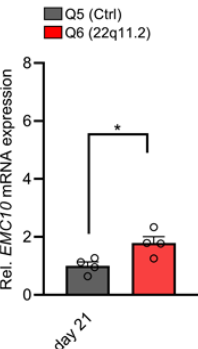

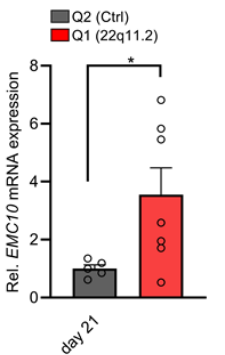

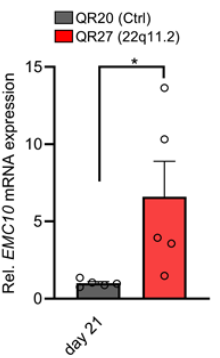


bioRxiv preprint doi: https://doi.org/10.1101/2022.03.01.482495; this version posted March 4, 2022. The copyright holder for this preprint (which was not certified by peer review) is the author/funder. All rights reserved. No reuse allowed without permission.

a

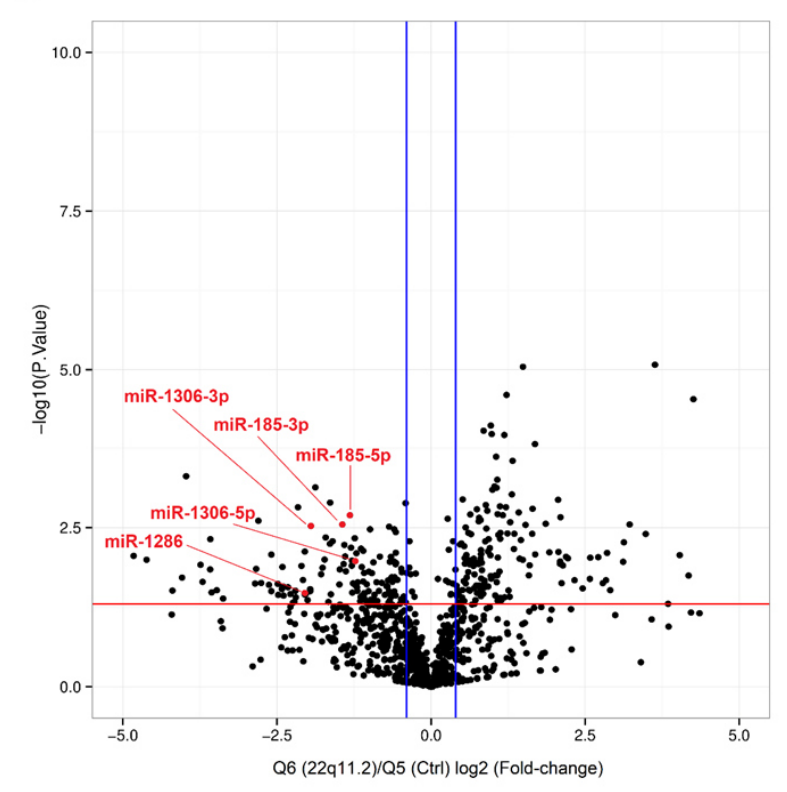

b

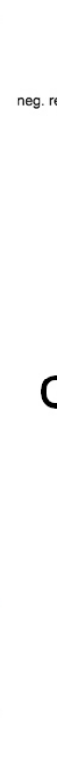

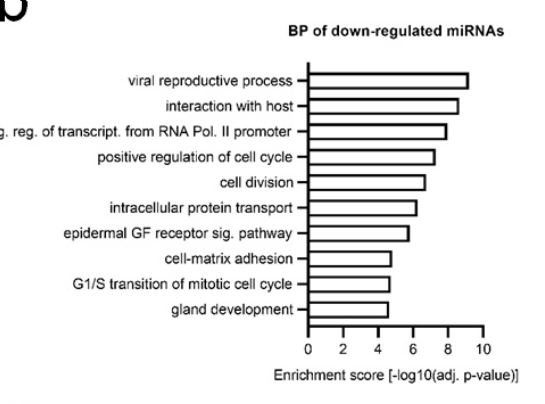

C

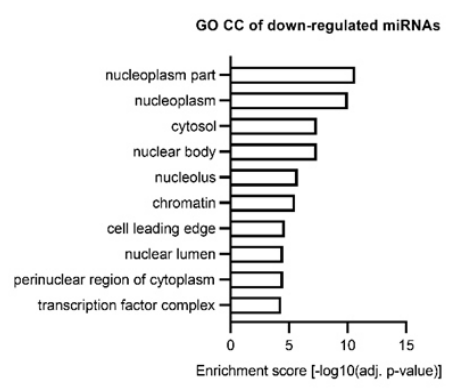

GO BP of up-regulated miRNAs
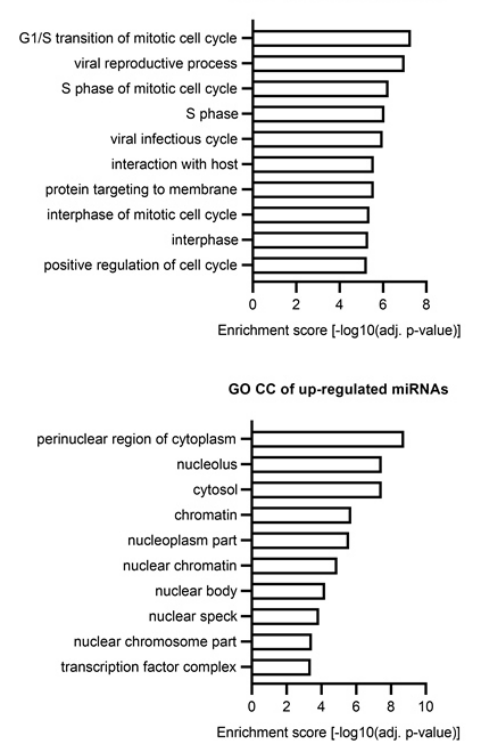

d

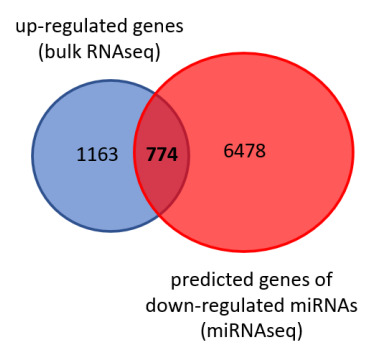

g

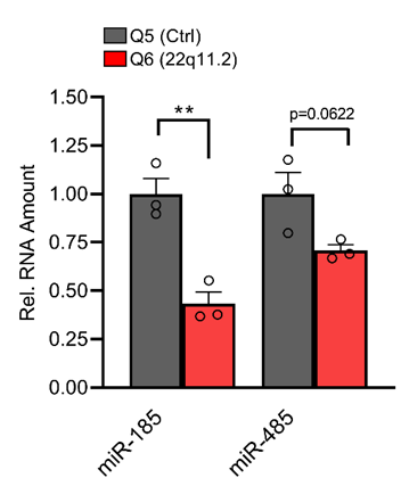

e

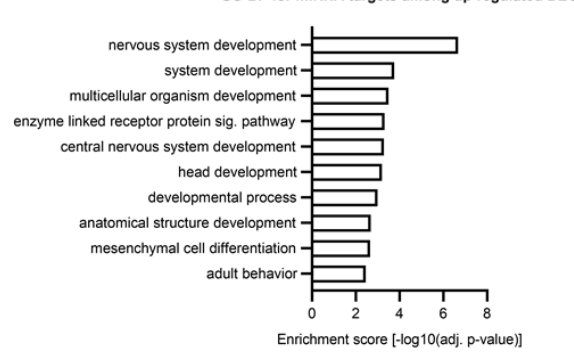

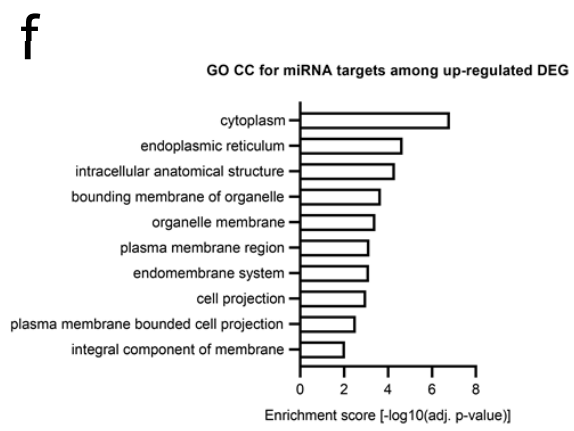

h

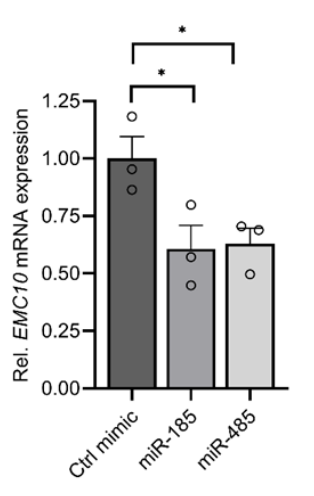

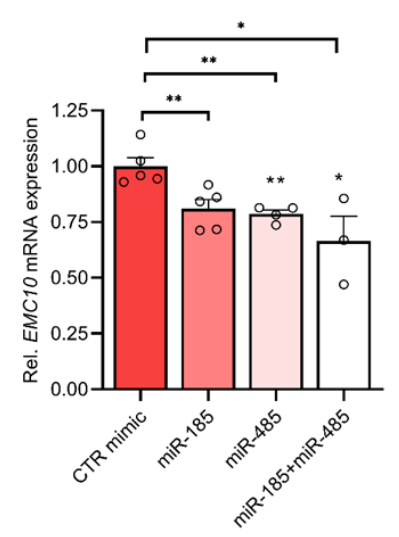

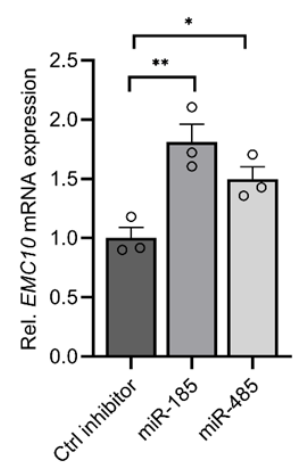


bioRxiv preprint doi: https://doi.org/10.1101/2022.03.01.482495; this version posted March 4, 2022. The copyright holder for this preprint (which was not certified by peer review) is the author/funder. All rights reserved. No reuse allowed without permission.

a

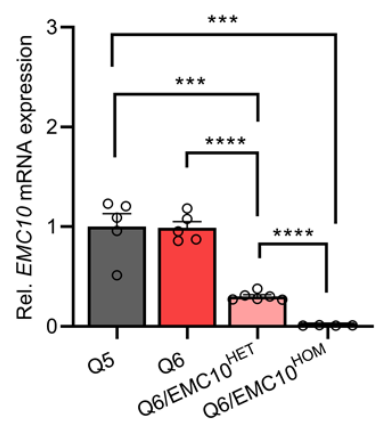

C
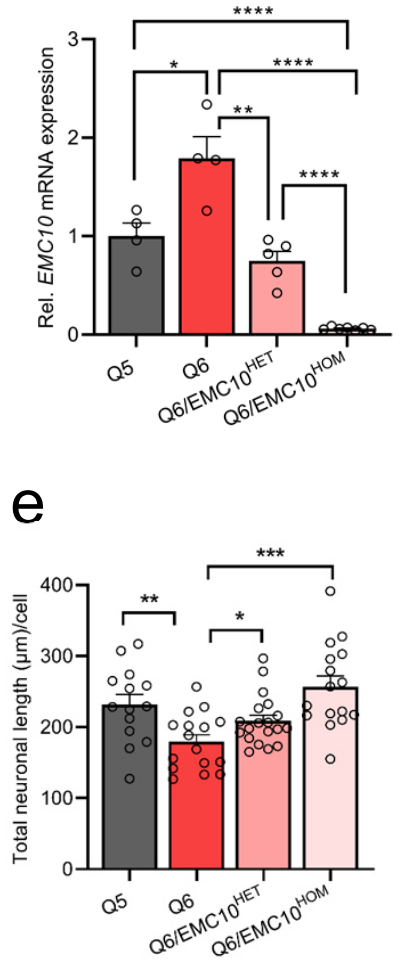

i

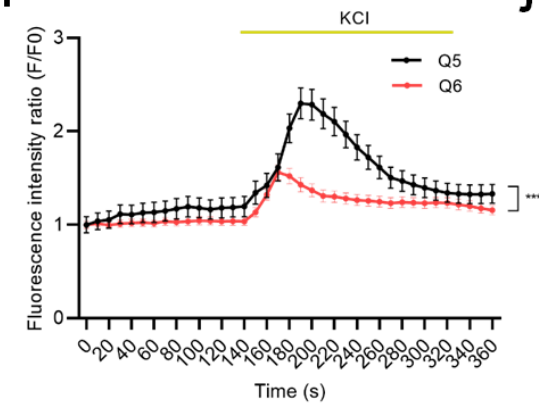

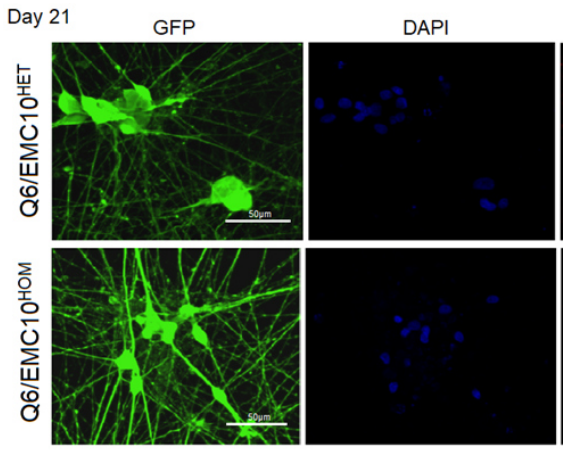

d

Q5

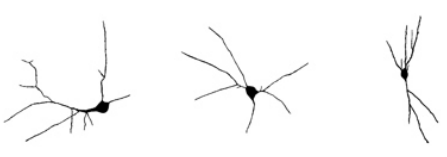

Q6/EMC10 HET

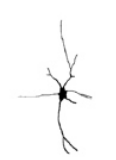

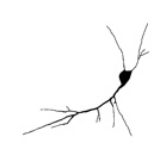
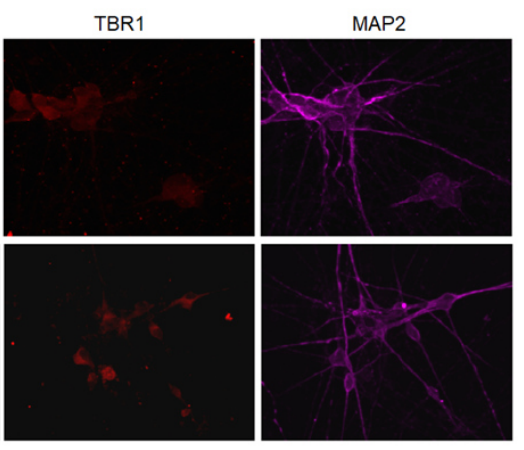

Q6

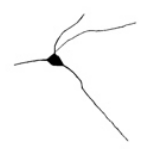

Q6/EMC10HOM
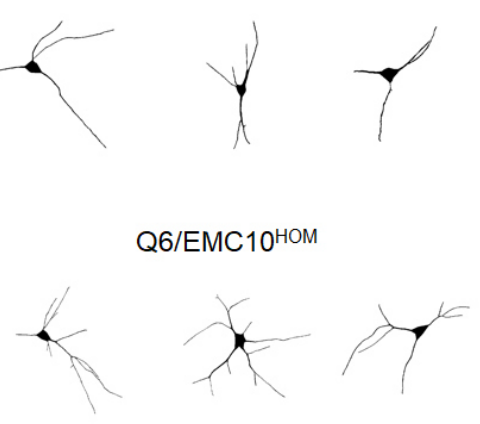

f

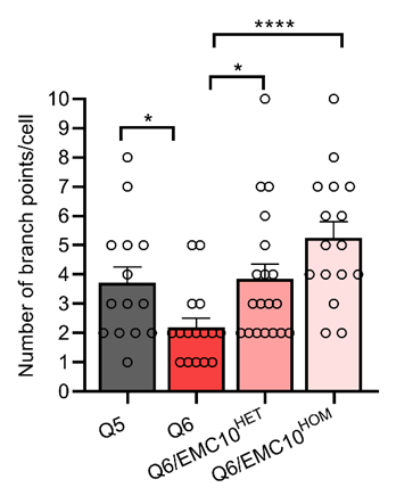

g

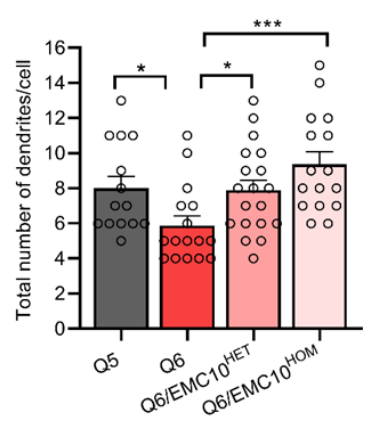

k

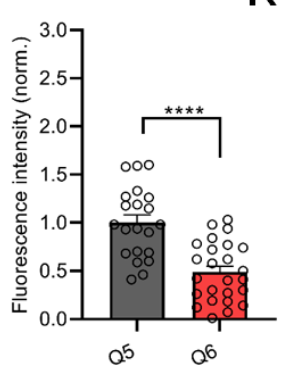

h

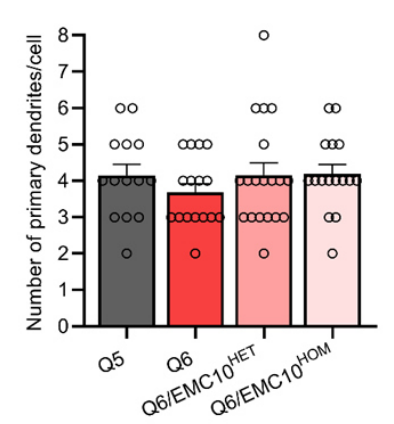

I
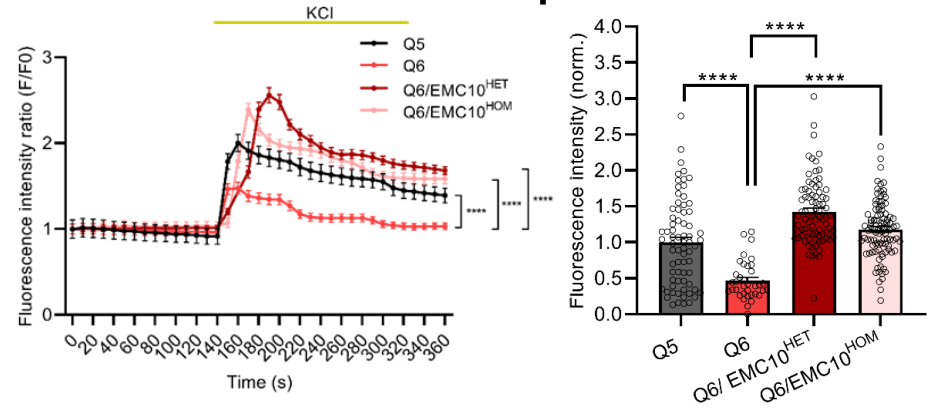

Figure 3 
bioRxiv preprint doi: https://doi.org/10.1101/2022.03.01.482495; this version posted March 4, 2022. The copyright holder for this preprint (which was not certified by peer review) is the author/funder. All rights reserved. No reuse allowed without permission.

a

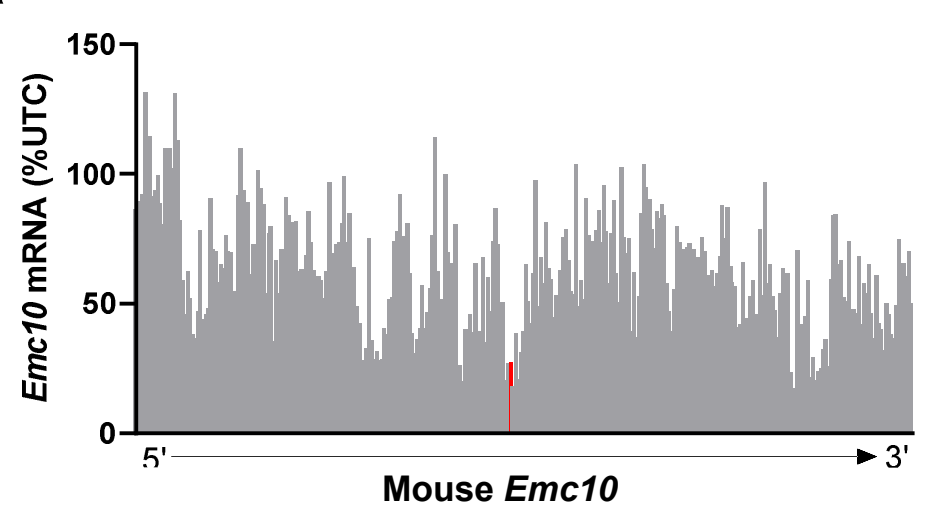

b

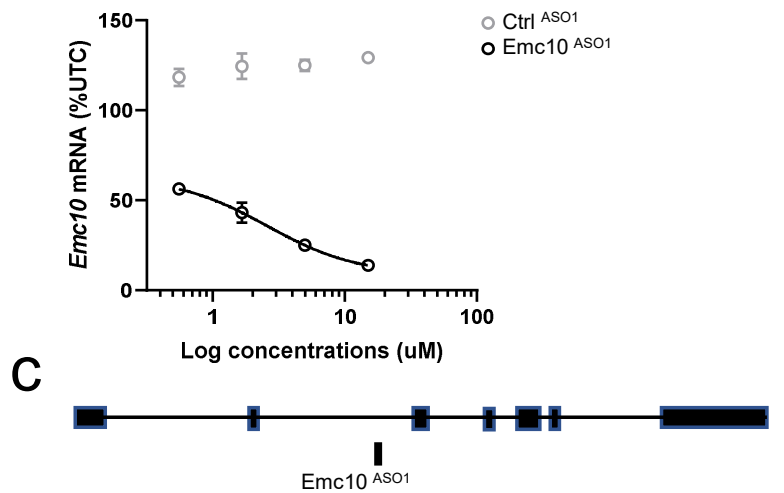

d

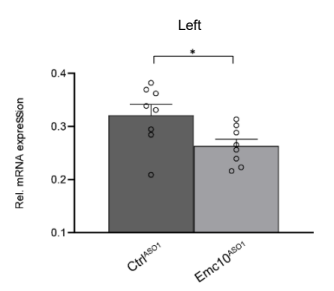

e

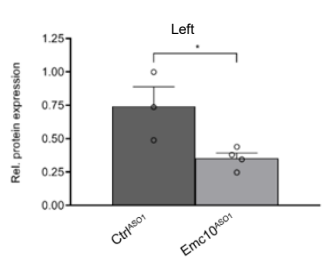

f

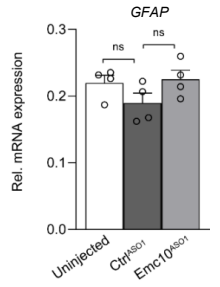

h

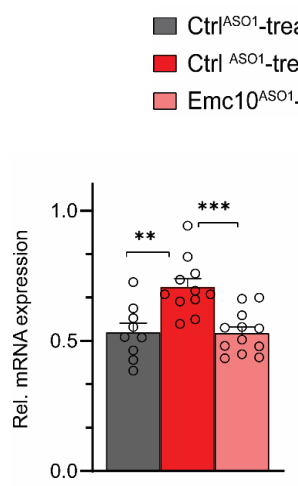

HPC
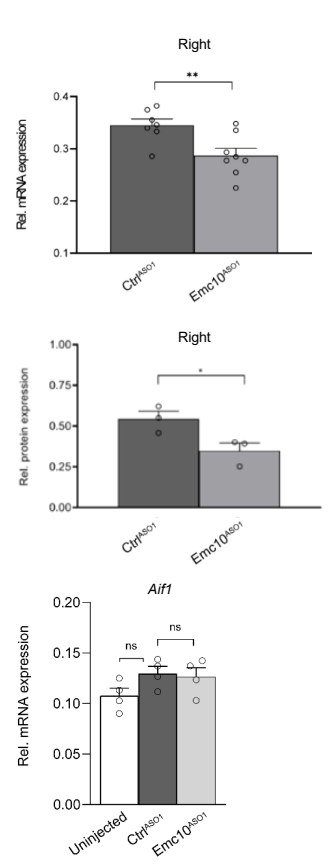

WT

$D f(16) A^{+/}$

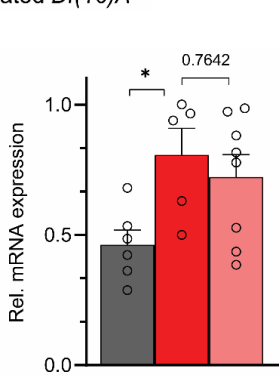

PFC g
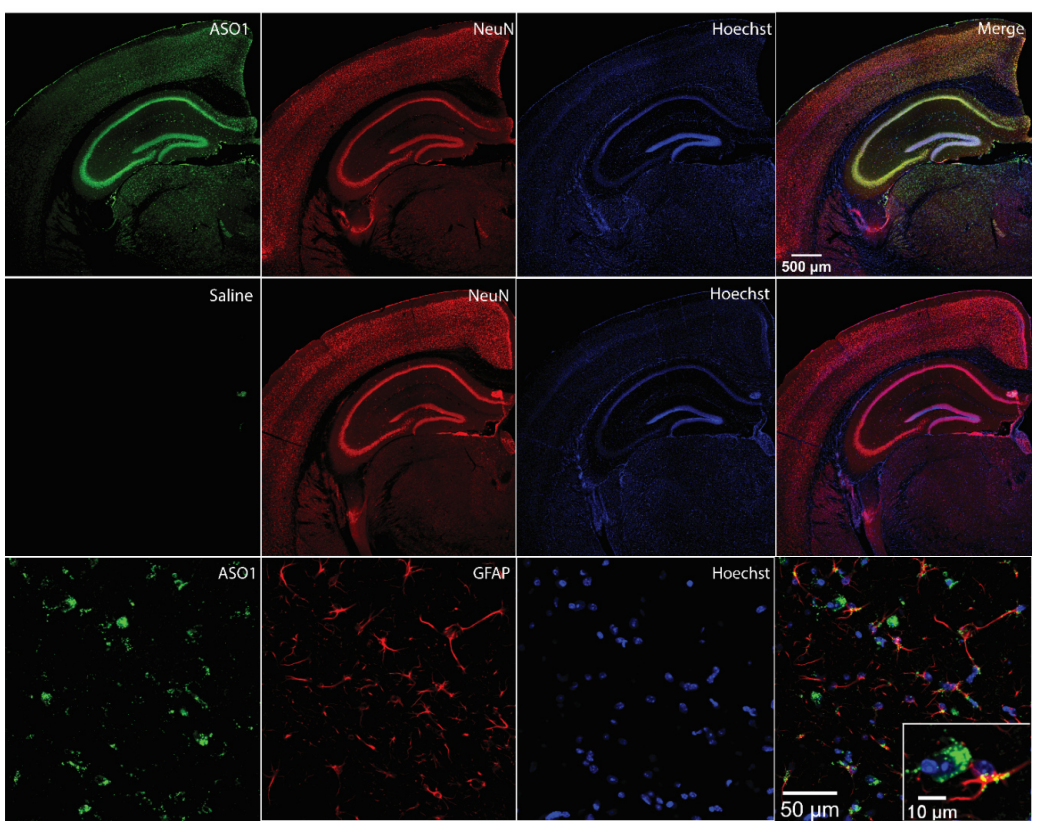

$\Delta$ 22q11.2 Genes

$\begin{array}{ll}\text { Genelist } & \text { Emc10 } \text { ASO1_treated }^{\text {ncRNA }} \\ \triangle \text { Emc10 } & \text { WT vs. Df(16)A }{ }^{+-}\end{array}$
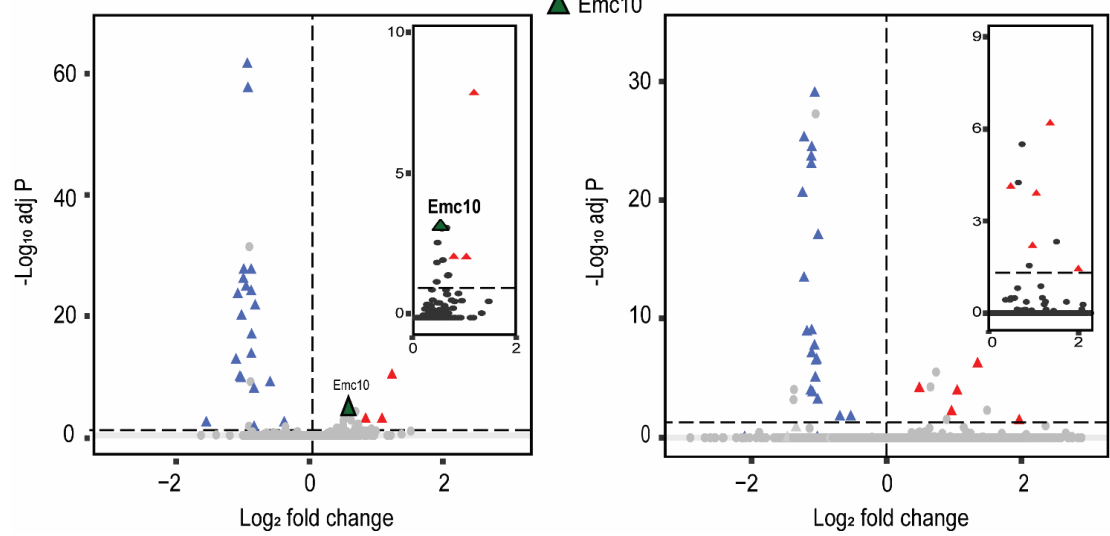

Figure 4 
bioRxiv preprint doi: https://doi.org/10.1101/2022.03.01.482495; this version posted March 4, 2022. The copyright holder for this preprint (which was not certified by peer review) is the author/funder. All rights reserved. No reuse allowed without permission.

a

C
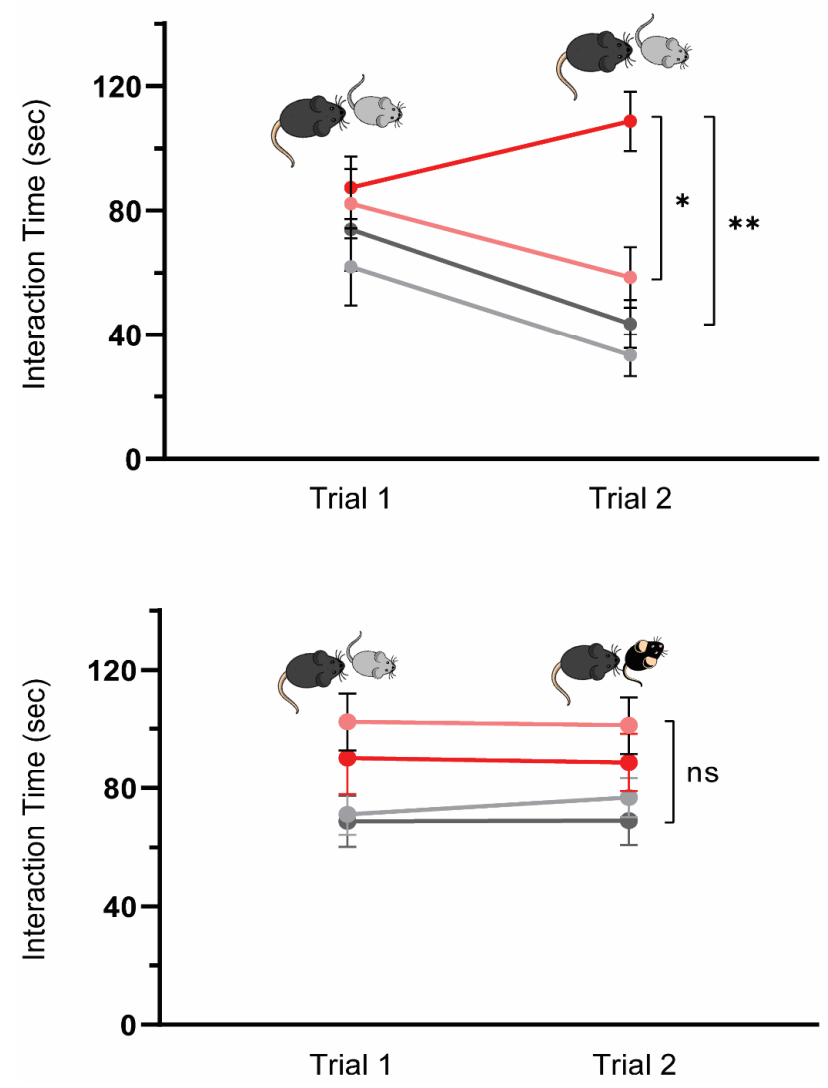

Day 1

\section{e}

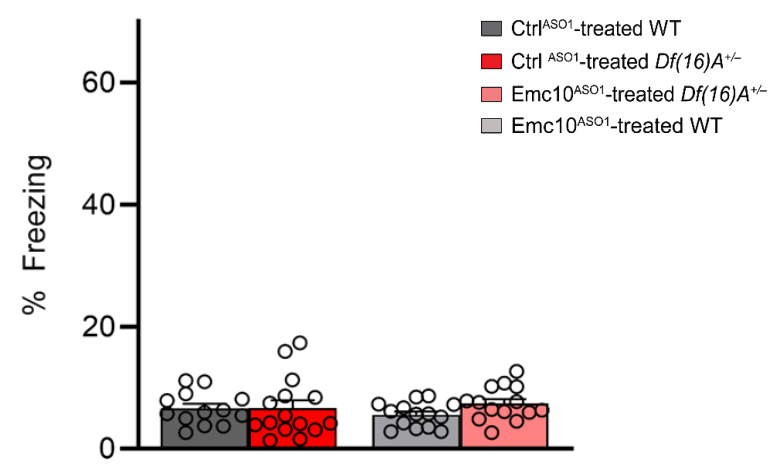

b

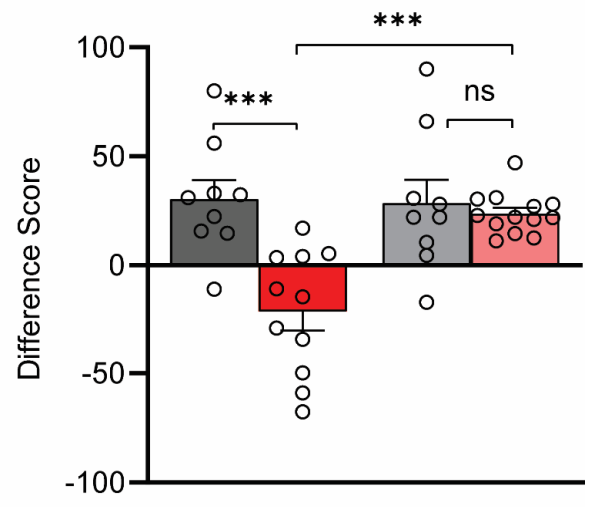

d

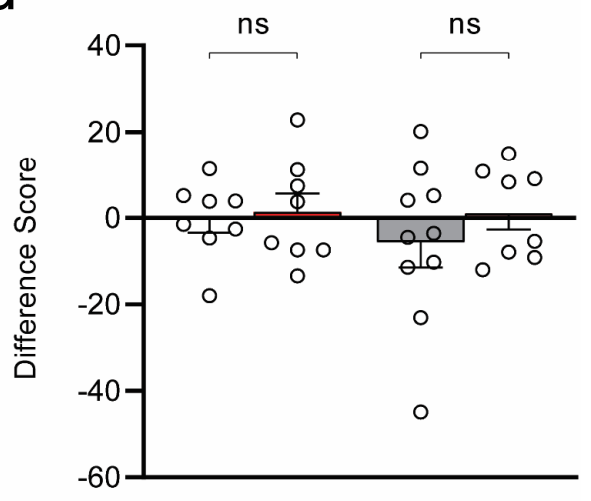

Day 2

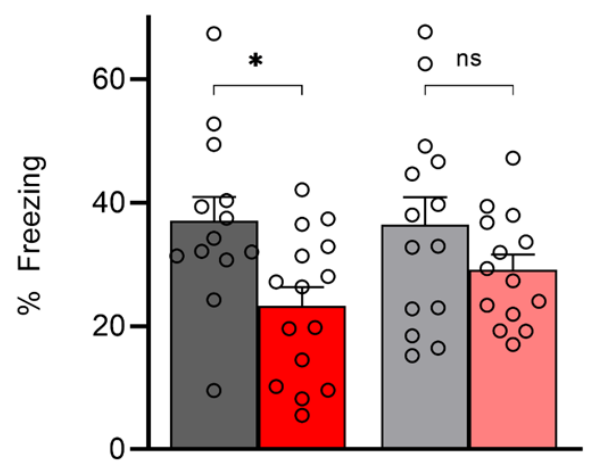


bioRxiv preprint doi: https://doi.org/10.1101/2022.03.01.482495; this version posted March 4, 2022. The copyright holder for this preprint (which was not certified by peer review) is the author/funder. All rights reserved. No reuse allowed without permission.

a

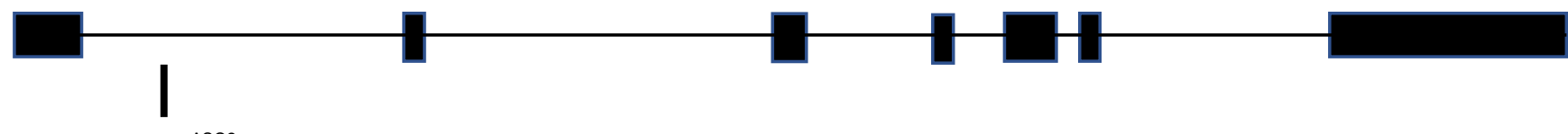

Emc10 ASO2

b
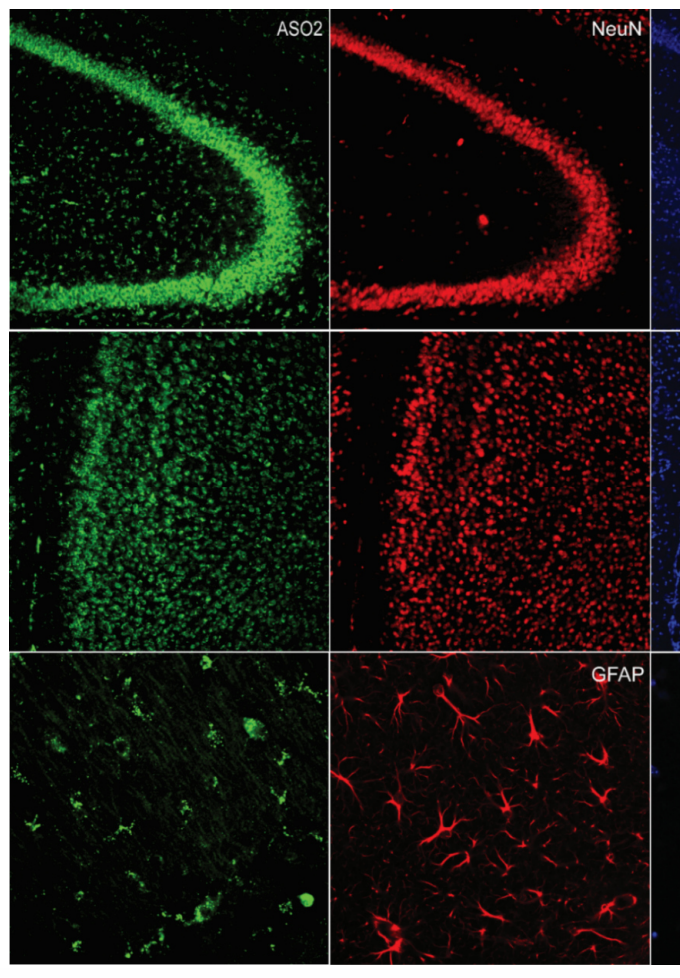

\section{f}

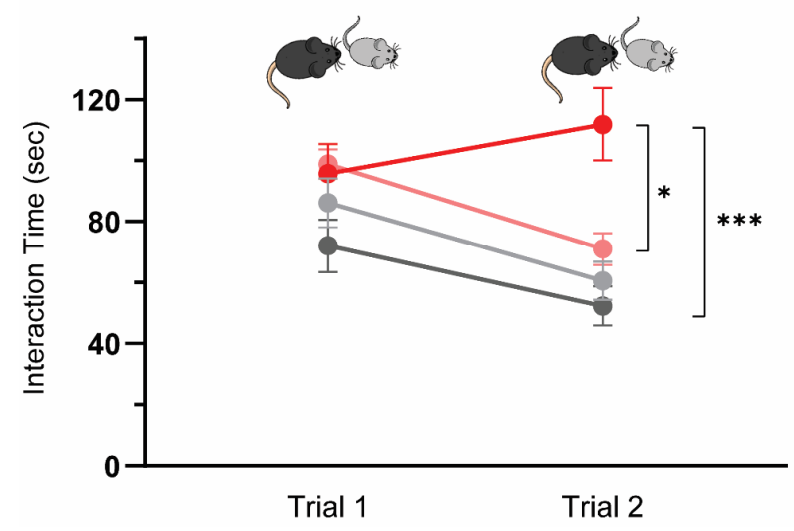

C

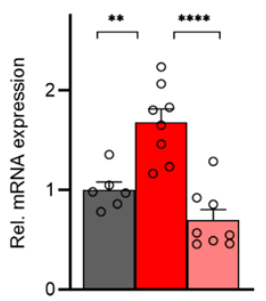

d

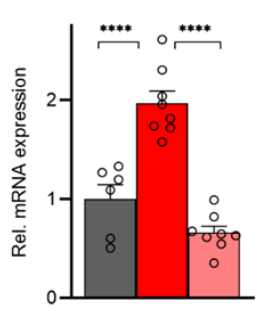

e

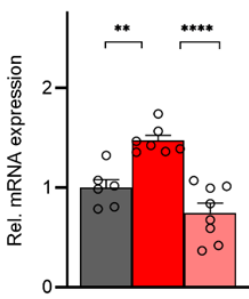

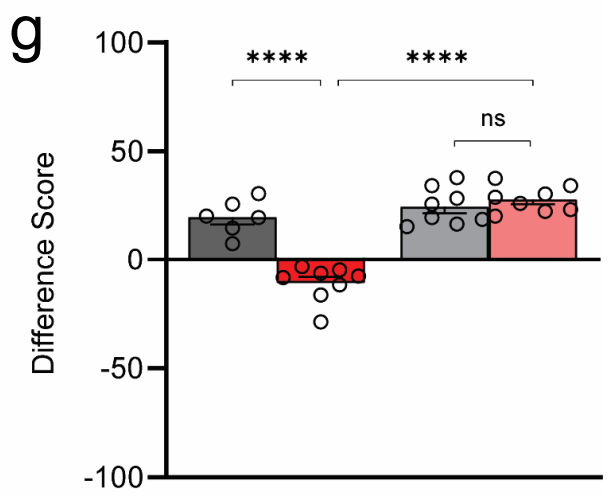

$\square \mathrm{Ctrl}{ }^{\mathrm{ASO2}}$-treated WT

Ctrl ASO2-treated $D f(16) A^{+1 /}$

$\square$ Emc10 $10^{A S O 2}$-treated $D f(16) A^{+\prime \prime}$

$\square$ Emc10 ${ }^{4 \text { sO2}_{2}}$-treated WT 
bioRxiv preprint doi: https://doi.org/10.1101/2022.03.01.482495; this version posted March 4, 2022. The copyright holder for this preprint (which was not certified by peer review) is the author/funder. All rights reserved. No reuse allowed without permission.

a Q5 (Ctrl)

Q6 (22q11.2)

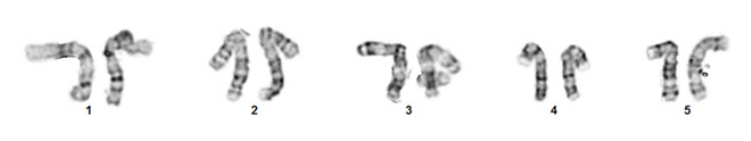

If in If y II II

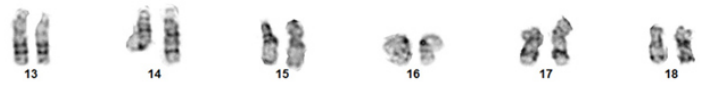

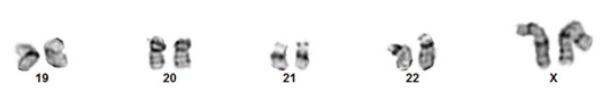

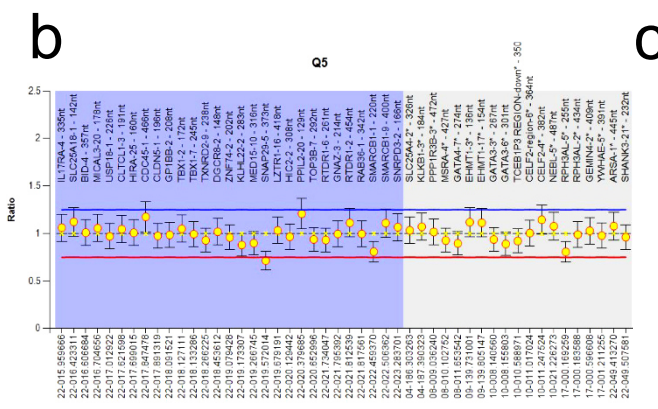

C

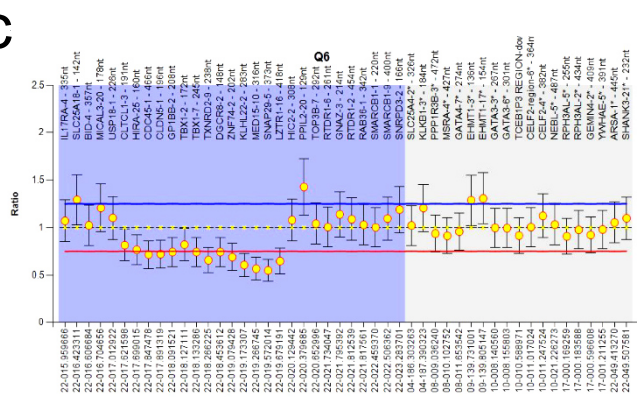

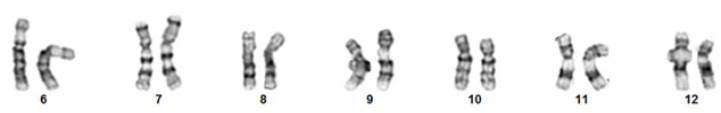

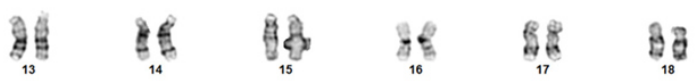

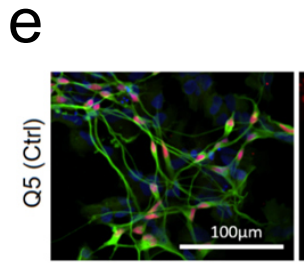

TBR1

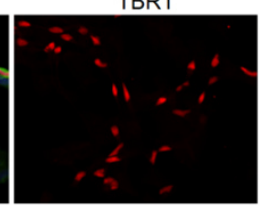

TUJ1

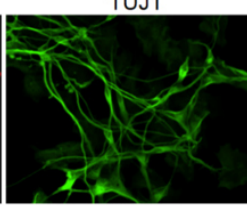

DAPI

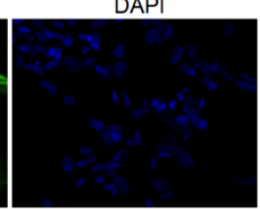

d

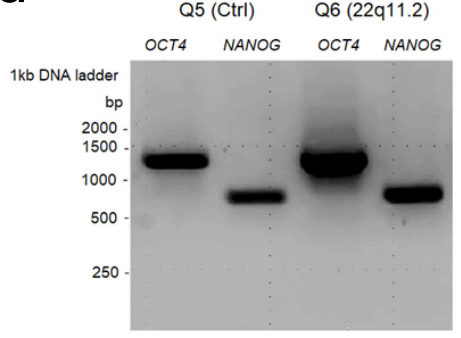

g
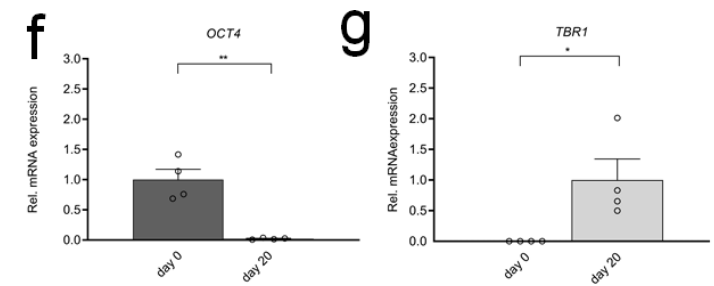
bioRxiv preprint doi: https://doi.org/10.1101/2022.03.01.482495; this version posted March 4, 2022. The copyright holder for this preprint (which was not certified by peer review) is the author/funder. All rights reserved. No reuse allowed without permission.

a

Expression of 22q11.2 region genes

DGCR2

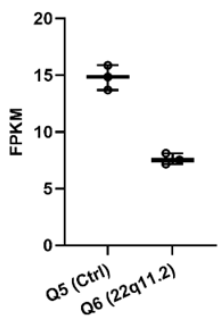

CDC45

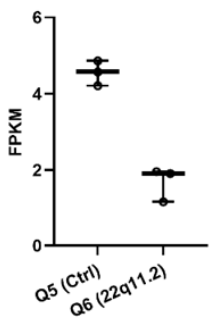

RANBP1

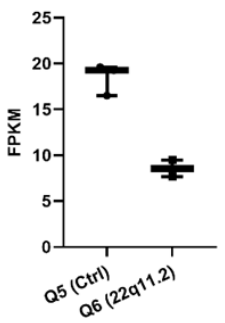

SNAP29

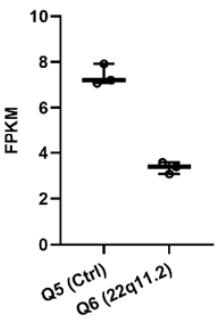

DGCR11

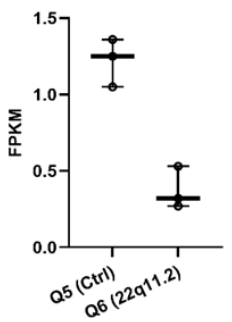

GNB1L

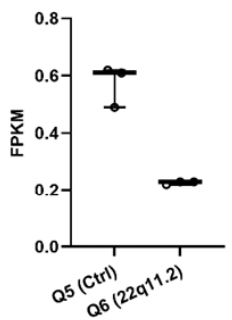

RTN4R

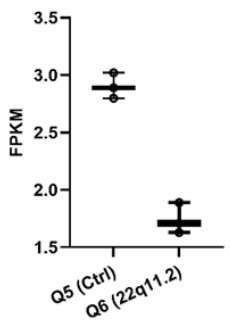

CRKL

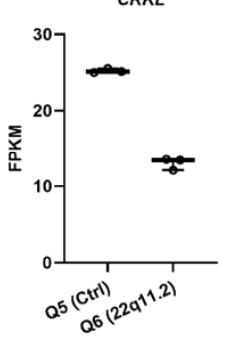

$S L C 25 A 1$

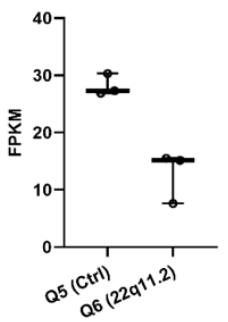

ARVCF

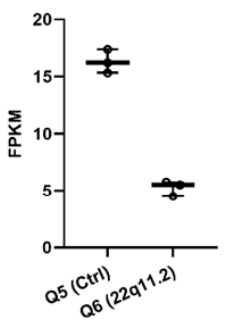

ZNF74

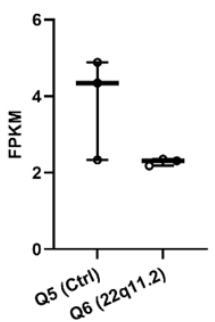

LZTR1

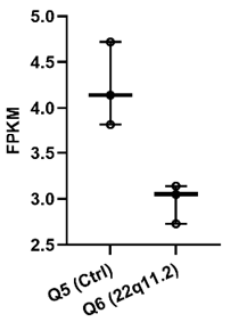

CLTCL1

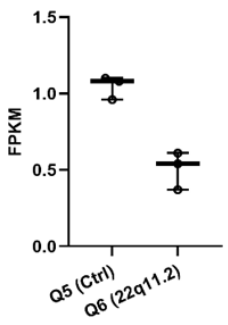

TANGO2

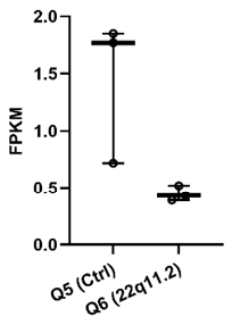

KLHL22

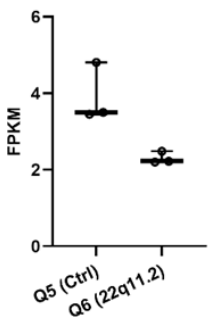

THAP7

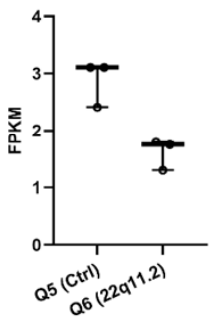

HIRA

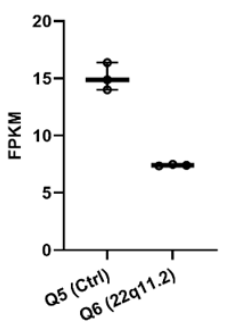

DGCR8

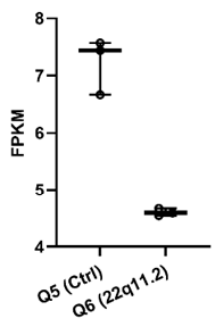

MED15
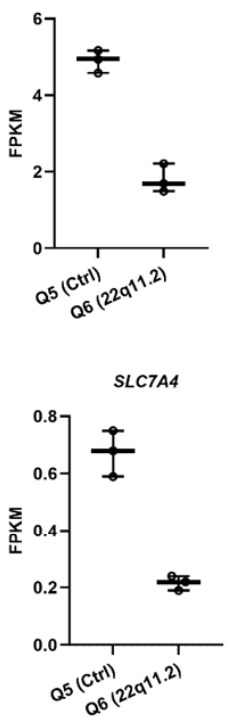

C22orf39

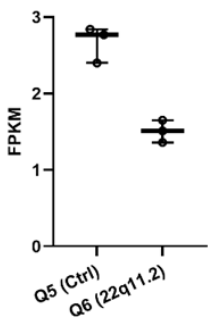

TRMT2A
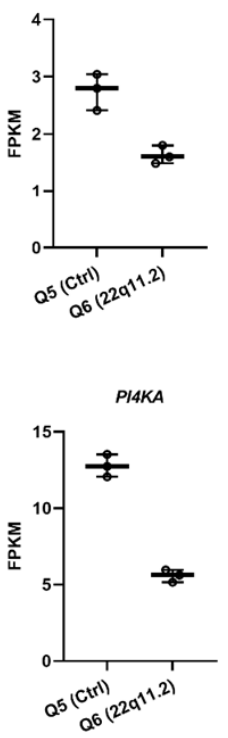

b

Expression of $19 q 13$ region gene EMC10

EMC10

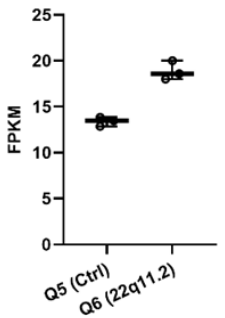

Figure S2 
bioRxiv preprint doi: https://doi.org/10.1101/2022.03.01.482495; this version posted March 4, 2022. The copyright holder for this preprint (which was not certified by peer review) is the author/funder. All rights reserved. No reuse allowed without permission.

a

Expression of 22q11.2 region miRNAs
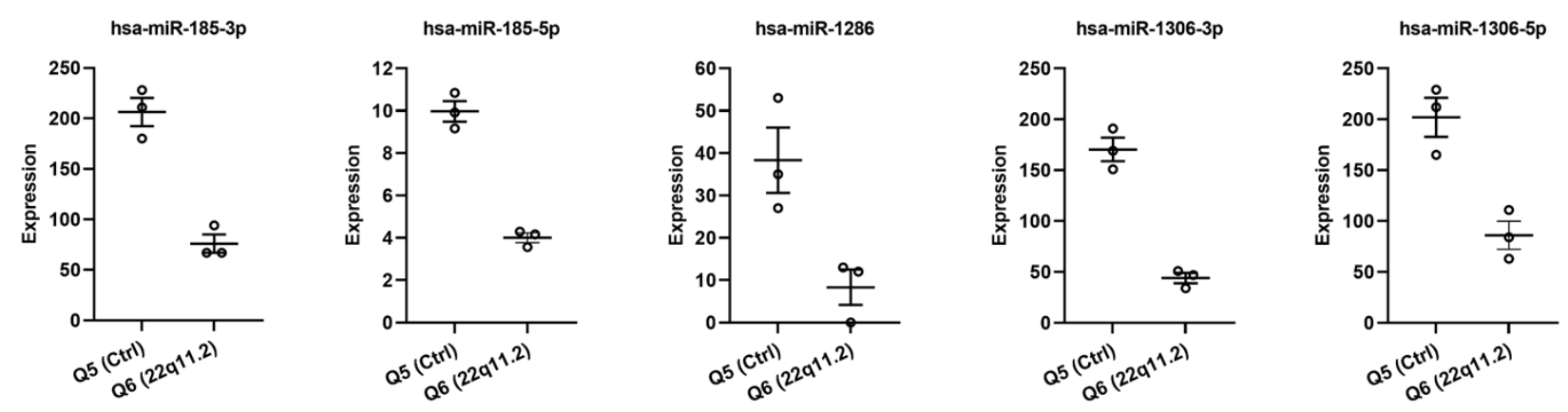

b

Expression of miRNAs outside the 22q11.2 region
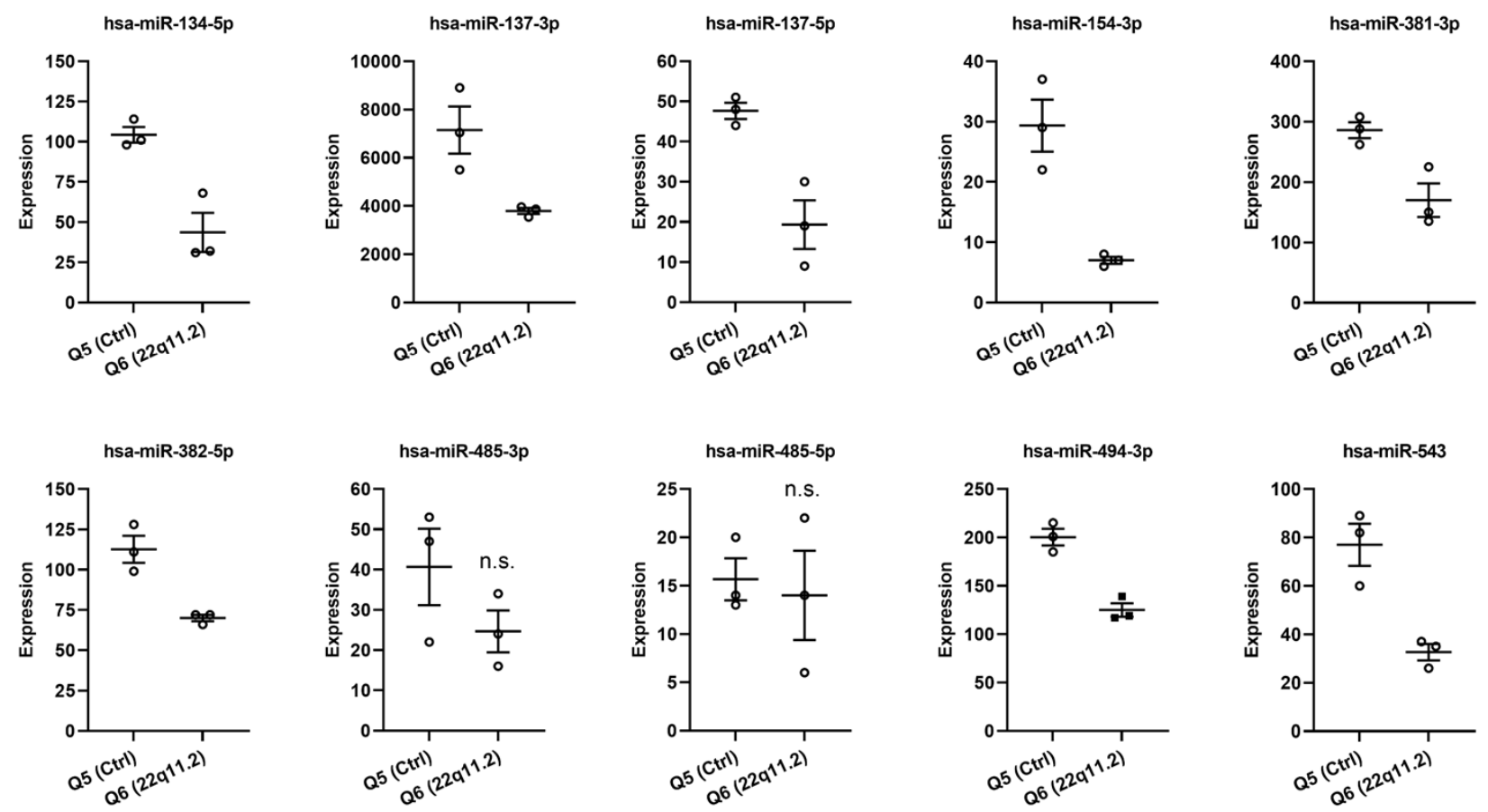
bioRxiv preprint doi: https://doi.org/10.1101/2022.03.01.482495; this version posted March 4, 2022. The copyright holder for this preprint (which was not certified by peer review) is the author/funder. All rights reserved. No reuse allowed without permission.

a

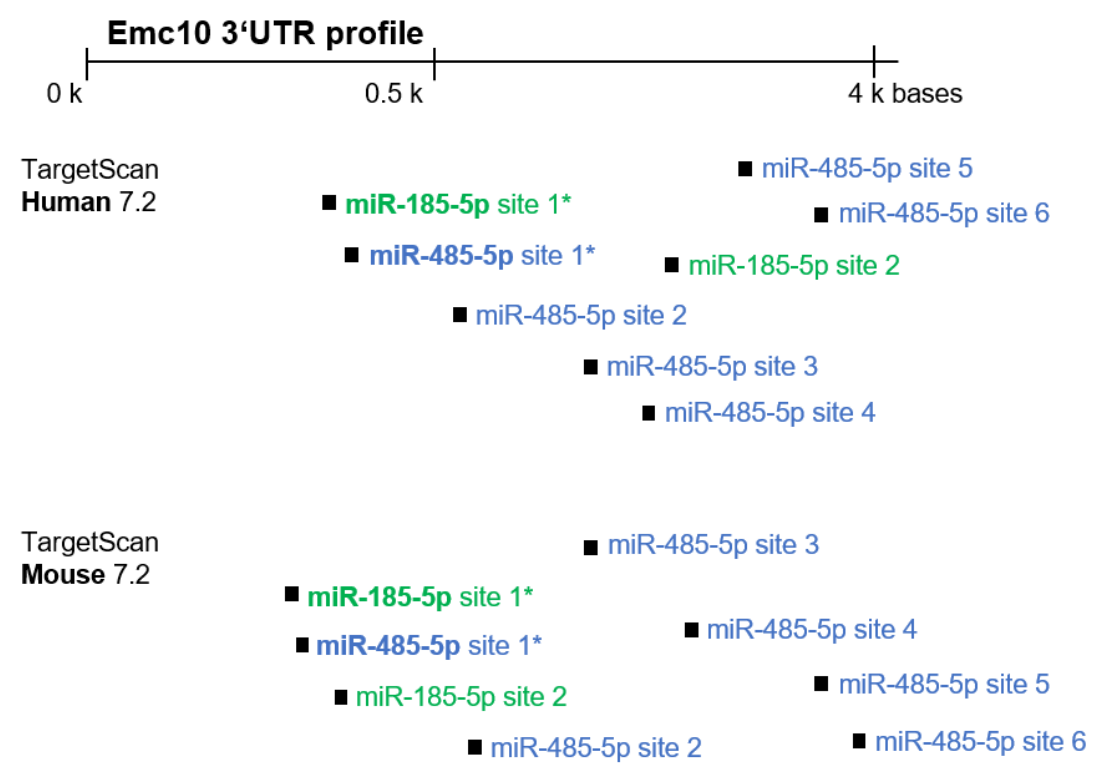

*conserved sites among mammals

b

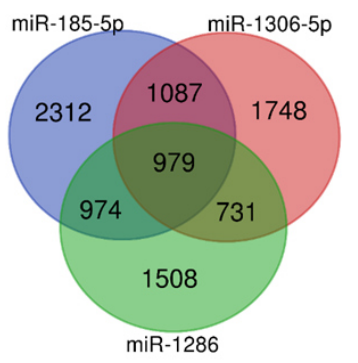

C

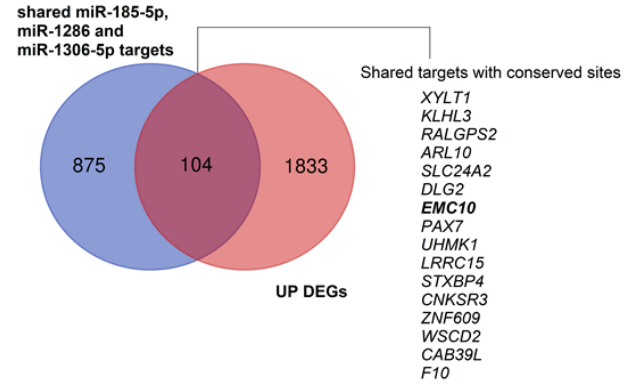


bioRxiv preprint doi: https://doi.org/10.1101/2022.03.01.482495; this version posted March 4, 2022. The copyright holder for this preprint (which was not certified by peer review) is the author/funder. All rights reserved. No reuse allowed without permission.

a EMC10 (transcript 201)

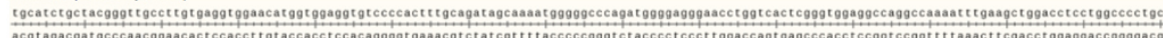

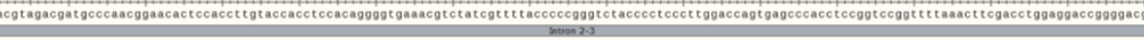

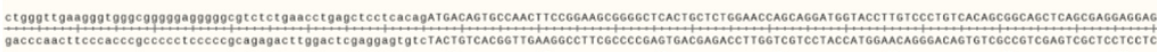

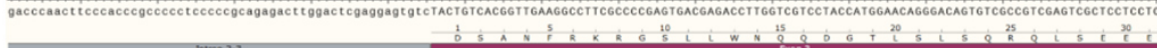

Cisarecios

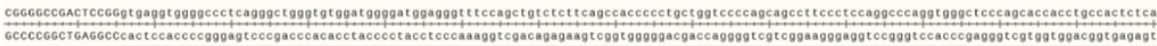

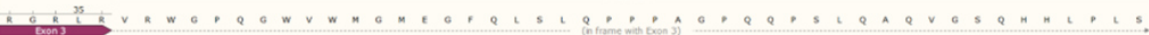

Q6/EMC10HET

C1982-NGSB-G1-CLONE1_7209_62.4

C1982-NGSB-G1-CLONE1_4335_37.6

AACCTGAGCTCCTCACAGATGACAGTGCCAACTTCC-..--GGCTCACTGCTCTGGA

C1982-NGSB_ref

AACCTGAGCTCCTCACAGATGACAGTGCCAACTTCCGGAAGCGGGGCTCACTGCTCTGGA

AACCTGAGCTCCTCACAGATGACAGTGCCAACTTCCGGAAGCGGGGCTCACTGCTCTGGA

Q6/EMC10 HOM

C1982-NGSB-G2-CLONE2_10075_43.1

C1982-NGSB-G2-CLONE2_13286_56.9

C1982-NGSB_ref

ACCAG-..--- CaGTACCTTGTCCCCTGTCACAGCGGCAGCTCAGCGAGGAGGAGCGGGGC ....-TGGTACCTTGT-CCCTGTCACAGCGGCAGCTCAGCGAGGAGGAGCGGGGC ACCAGCaggaTGGTACCTTGT-CCCTGTCACAGCGGCAGCTCAGCGAGGAGGAGCGGGGC

$\frac{\text { C1982-EMC10-92 }}{2-C C C}$

b

b

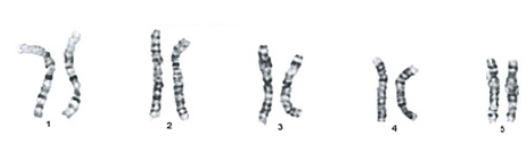

5ु

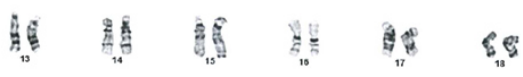

Q6/EMC10 HOM

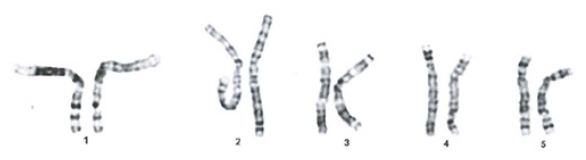

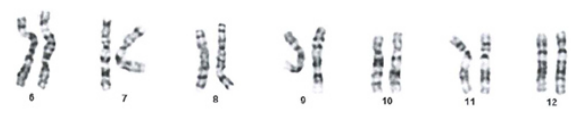

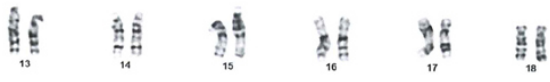

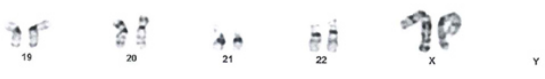

C

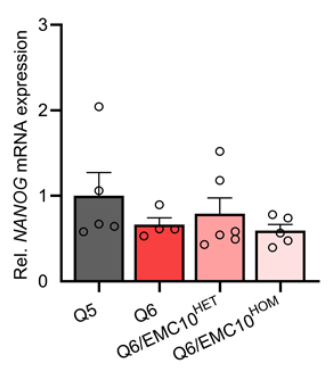

d

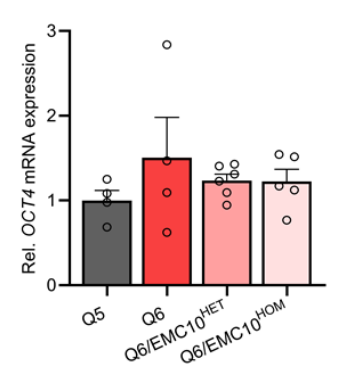

$\mathrm{e}$

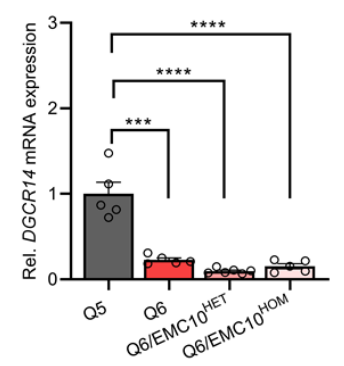

g

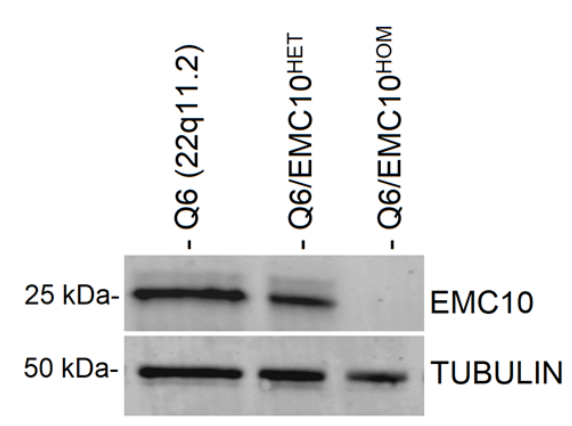


bioRxiv preprint doi: https://doi.org/10.1101/2022.03.01.482495; this version posted March 4, 2022. The copyright holder for this preprint (which was not certified by peer review) is the author/funder. All rights reserved. No reuse allowed without permission.

a

$$
\text { Emc10 Cortex }
$$

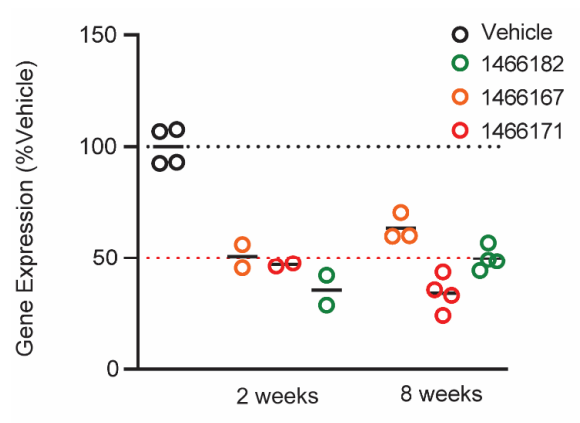

b

Emc10 TSC

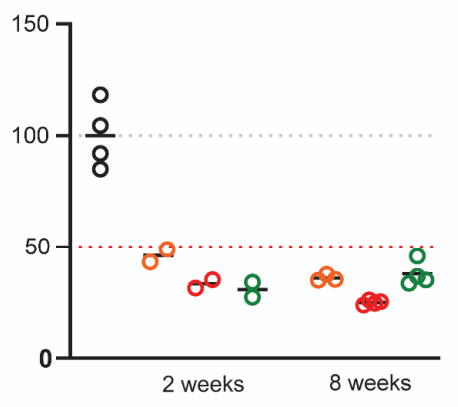

C

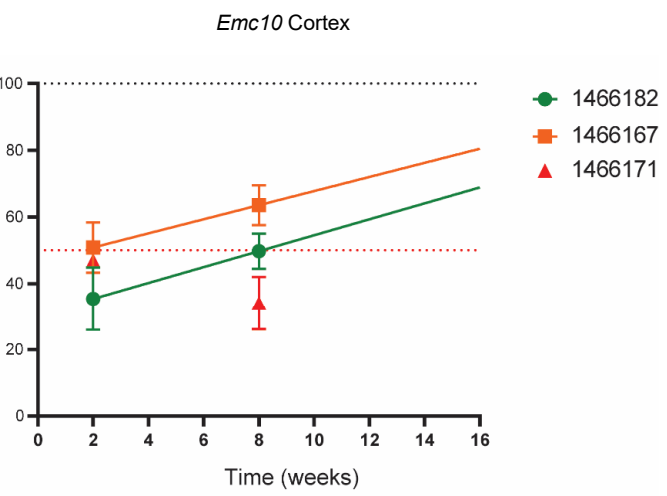

Figure S6 
bioRxiv preprint doi: https://doi.org/10.1101/2022.03.01.482495; this version posted March 4, 2022. The copyright holder for this preprint (which was not certified by peer review) is the author/funder. All rights reserved. No reuse allowed without permission.

\section{Supplementary Table S1: Clinical and demographic characteristics of 22q11.2DS/SCZ patients and healthy controls.}

\begin{tabular}{|c|c|c|c|}
\hline Columbia ID & Ruttgers University ID & Gender & Diagnosis \\
\hline QR20 (1) & MH0159020 & male & none \\
QR27 (1) & MH0159027 & male & schizoaffective disorder/22q11.2DS \\
Q1 $\left(2^{*}\right)$ & - & male & schizoaffective disorder/22q11.2DS \\
Q2 $\left(2^{*}\right)$ & - & male & none \\
Q5 $\left(2^{* *}\right)$ & - & female & none \\
Q6 $\left(2^{* *}\right)$ & - & female & early onset schizophrenia/22q11.2DS \\
\hline
\end{tabular}

1 hiPSC lines were obtained from the Stem Cell Respository of National Institute of Mental Health

2 hiPSC lines were obtained from the Ciolumbia Stem Cell Core Facility

*sibling pair

${ }^{* *}$ twin pair 
bioRxiv preprint doi: https://doi.org/10.1101/2022.03.01.482495; this version posted March 4, 2022. The copyright holder for this preprint (which was not certified by peer review) is the author/funder. All rights reserved. No reuse allowed without permission.

\section{Supplementary Table S2: Predicted targets of miR-185-5p, miR-1286 and miR-1306-5p} in EMC10 3'UTR.

\begin{tabular}{|c|cc|cc|cc|}
\hline EMC10 & hsa-miR-185-5p & $\begin{array}{c}\text { predicted } \\
\text { occupancy }\end{array}$ & hsa-miR-1306-5p & $\begin{array}{c}\text { predicted } \\
\text { occupancy }\end{array}$ & $\begin{array}{c}\text { predicted } \\
\text { hsa-miR-1286 }\end{array}$ & $\begin{array}{c}\text { occupancy } \\
\text { occule }\end{array}$ \\
\hline conserved site & $\mathbf{8 m e r ^ { * }}$ & $\mathbf{0 . 2 0 9 1}$ & - & - & - & - \\
\hline $\begin{array}{c}\text { poorly conserved } \\
\text { site }\end{array}$ & 7 mer-A1** & 0.2091 & $7 m e r-m 8^{* *}$ & 0.0082 & $7 m e r-A 1^{* * *}$ & 0.0189 \\
& - & - & - & - & $7 m e r-A 1^{* * *}$ & 0.0189 \\
& - & - & - & - & $7 m e r-A 1^{* * *}$ & 0.0189 \\
\hline
\end{tabular}

*conserved miRNA binding sites among mammals

${ }^{* *}$ poorly conserved miRNA binding sites among human, chimp and rhesus

${ }^{* * *}$ poorly conserved miRNA binding sites among human and chimp 


\section{Supplementary Table S3: List of primer sequences used for qRT-PCRs.}

\begin{tabular}{|c|l|l|}
\hline Target & \multicolumn{1}{|c|}{ Forward Primer } & \multicolumn{1}{c|}{ Reverse Primer } \\
\hline U6 snRNA & CTCGCTTCGGCAGCACA & AACGCTTCACGAATTTGCGT \\
\hline pre-miR-134 & TGTGACTGGTTGACCAGAGGG & GGTGACTAGGTGGCCCACAG \\
\hline pre-miR-137 & GGTGACGGGTATTCTTGGGT & CGACTACGCGTATTCTTAAGCAAT \\
\hline pre-miR-185 & GCGAGGGATTGGAGAGAAAG & GAGGGAAGGACCAGAGGAAA \\
\hline pre-miR-212 & CACCTTGGCTCTAGACTGCTT & GCCGTGACTGGAGACTGTTA \\
\hline pre-miR-377 & TTGAGCAGAGGTTGCCCT & CAAACAAAAGTTGCCTTTGTGT \\
\hline pre-miR-485 & GAGAGGCTGGCCGTGATG & GACACTAGAAGAGAGGAGAG \\
\hline NANOG & TTTGTGGGCCTGAAGAAAACT & AGGGCTGTCCTGAATAAGCAG \\
\hline OCT4 & ACAACAATGAGAACCTTCAGGAG & GCCGGTTACAGAACCACACT \\
\hline
\end{tabular}


Supplementary Table S4: List of genes upregulated in CtrlASO1 treated- Df16(A)+/- mice compared to CtrlASO1 treated-WT mice but not in the Emc10ASO1 treated Df16(A)+/compared to Emc10ASO1 treated-WT mice.

\begin{tabular}{|l|l|l|}
\hline \multicolumn{1}{|c|}{ Gene } & \multicolumn{1}{|c|}{ Chromosome } & \multicolumn{1}{c|}{ Description } \\
\hline Emc10 & 7 & ER Membrane protein Complex subunit 10 \\
\hline Mir9-3hg & 7 & Mir9-3 host gene \\
\hline Plxnd1 & 6 & Plexin D1 \\
\hline Cd68 & 11 & CD68 antigen \\
\hline Gm28439 & 1 & Predicted gene 28439 \\
\hline Mir22hg & 11 & Mir22 host gene (non-protein coding) \\
\hline Adgre4 & 17 & Adhesion G protein-coupled receptor E4 \\
\hline Tnn & 1 & Tenascin N \\
\hline
\end{tabular}

